\title{
Teichmüller theory and handle addition for minimal surfaces
}

\author{
By Matthias Weber and Michael WolF*
}

1. Introduction

1.1. The surfaces

1.2. The proof

2. Background, notation and a sketch of the argument

2.1. Minimal surfaces

2.2. Teichmüller theory

2.3. A brief outline of the proof

3. The geometry of orthodisks

3.1. Orthodisks

3.2. From orthodisks to Riemann surfaces

3.3. From orthodisks to Weierstrass data

3.4. Geometric significance of the formal Weierstrass data

3.5. Examples of simple orthodisks

3.6. Orthodisks for the Costa towers

3.7. More orthodisks by drilling holes

4. The space of orthodisks

4.1. Introduction

4.2. Geometric coordinates for the $\mathrm{DH}_{1,1}$-surface

4.3. Height function for the $\mathrm{DH}_{1,1}$-surface

4.4. Geometric coordinates for the $\mathrm{DH}_{m, n}$-surfaces

4.5. Height functions for the $\mathrm{DH}_{m, n}$-surfaces

4.6. Properness of the height functions for the $\mathrm{DH}_{m, n}$-surfaces

4.7. A monodromy argument

5. The gradient flow

5.1. Overall strategy

5.2. Deformations of $\mathrm{DH}_{1,1}$

5.3. Infinitesimal pushes

6. Regeneration

7. Nonexistence of the $\mathrm{DH}_{m, n}$-surfaces with $n<m$

*The second author was partially supported by NSF grant number DMS-9626565 and the SFB. 
8. Extensions and generalizations

8.1. Higher symmetry

8.2. Deformations with more catenoidal ends

8.3. Embeddedness aspects of $\mathrm{DH}_{m, n}$

9. References

\section{Introduction}

In this paper, we develop Teichmüller theoretical methods to construct new minimal surfaces in $\mathbb{E}^{3}$ by adding handles and planar ends to existing minimal surfaces in $\mathbb{E}^{3}$. We exhibit this method on an interesting class of minimal surfaces which are likely to be embedded, and have a low degree Gaußmap for their genus. In particular, we exhibit a two-parameter family of complete minimal surfaces in the Euclidean three-space $\mathbb{E}^{3}$; these surfaces are embedded (at least) outside a compact set and are indexed (roughly) by the number of ends they have and their genus. They have at most eight selfsymmetries despite being of arbitrarily large genus, and are interesting for a number of reasons. Moreover, our methods also extend to prove that some natural candidate classes of surfaces cannot be realized as minimal surfaces in $\mathbb{E}^{3}$. As a result of both aspects of this work, we obtain a classification of a family of surfaces as either realizable or unrealizable as minimal surfaces.

This paper is a continuation of the study we initiated in [WW]; in a strong sense it is an extension of that paper, as the essential organization of the proof, together with many details, have been retained. Indeed, part of our goal in writing this paper was a demonstration of the robustness of the methods of $[\mathrm{WW}]$, in that here we produce minimal surfaces of a very different character than those produced in [WW], yet the proof changes only in a few quite technical ways. (In particular, the present proof handles the previous case of Chen-Gackstatter surfaces of high genus as an elementary case.) Indeed in the intervening years between our initial preparation of this manuscript and its final revision for publication, this method has been applied to produce other families of surfaces of substantively different characteristics or to prove their nonexistence ([WW2], [MW]).

1.1. The surfaces. Hoffman and Meeks (see [Ho-Me]) have conjectured that any complete embedded minimal surface in space has genus at least $r-2$, where $r$ denotes the number of ends of the surface. In this paper, we provide significant evidence for this conjecture in the situation where the surfaces have eight symmetries. This is an important case for two reasons: first, it is presently unknown whether there are any complete embedded minimal sur- 
faces which have no symmetries ${ }^{1}$, and second, there are very few families of examples known where there are more than four ends. (Indeed, the only such constructions available are from the recent work of Kapouleas [Kap], where the genus is both high and inestimable.)

In particular, we consider two families of surfaces, with the first included in the second. The first case consists of surfaces $C T_{g}$ which generalize Costa's example [Cos]. We prove

Theorem A. For all odd genera $g$, there is a complete minimal surface $C T_{g} \subset \mathbb{E}^{3}$ which is embedded outside a compact surface with boundary of genus $g$, with $g$ parallel (horizontal) planar ends and two catenoid ends. The symmetry group of $C T_{g}$ is generated by reflective symmetries about a pair of orthogonal vertical planes and a rotational symmetry about a horizontal line.

These surfaces represent the borderline case for the conjecture. (The even genus cases have substantially different combinatorics, and require a different treatment.) Consider the Riemann surface underlying such an example: it is a fundamental theorem of Osserman [Oss1] that such a surface is conformally a compact surface of genus $g$, punctured at points corresponding to the ends. Let $Z$ denote the vertical coordinate of such a minimal surface: clearly, $Z$ is critical at the $g$ points corresponding to the planar ends, the two points corresponding to the catenoid ends, and $g$ interior points where the two reflective planes meet the surface.

We generalize these surfaces as follows, imagining Drilling additional Holes to obtain surfaces $\mathrm{DH}_{m, n}$ (see $\S 3.7$ ).

Theorem B. (i) For every pair of integers $n \geq m \geq 1$, there exists a complete minimal surface $\mathrm{DH}_{m, n} \subset \mathbb{E}^{3}$ of genus $m+n+1$ which is embedded outside a compact set with the following properties: it has $2 n+1$ vertical normals, $2 m+1$ planar ends, and two catenoid ends. The symmetry group is as in Theorem $\mathrm{A}$.

(ii) For $n<m$, there is no complete minimal surface with those symmetries of the type $\mathrm{DH}_{m, n}$ (and $2 n+1$ vertical normals, $2 m+1$ planar ends, and two catenoid ends).

In the second statement, the surfaces for which we prove nonexistence are in precise analogy with the surfaces for which we prove existence. There are many configurations of surfaces which have the given eight symmetries and $2 n+1$ vertical normals, $2 m+1$ planar ends, and two catenoid ends, and we will indicate the range of possible choices in $\S 3$ : in the nonexistence section,

\footnotetext{
${ }^{1}$ Added in proof. M. Traizet $[\mathrm{Tr}]$ has announced the proof of the existence of a complete embedded minimal surface with no symmetries.
} 
we concentrate only on the candidates which the rest of the paper indicates are most likely to exist. In particular, we do not prove a more general statement ruling out all surfaces of the rough description of having $2 n+1$ vertical normals, $2 m+1$ planar ends, and two catenoid ends (although many of the possible configurations we do not treat would also have no minimal representatives, with the proofs of nonexistence being precisely analogous to the proof we give in $\S 7$ ). We give a precise formulation of the statement of Theorem B(ii) at the outset of $\S 7$.

Theorem A follows from Theorem B (i) by setting $n=m=\frac{1}{2}(g-1)$. The weak embeddedness statement in Theorem B is strengthened somewhat in $\S 9$; we conjecture (supported by some numerical evidence) that these surfaces are, in fact, embedded. The restriction to planar ends is unnecessary: in $\S 8$ we show that these surfaces are deformable to having catenoid ends. Theorem B is displayed in tabular form at the end of $\S 3$. (In that table, we also add some information about the case $m=0$, which was excluded from the statement of Theorem B.)

In summary, for the case of "essentially embedded" surfaces (i.e. those surfaces which are embedded outside of a compact set) with eight symmetries and odd ends, the conjecture is robustly true: no counterexamples (of the type $\left.\mathrm{DH}_{m, n}\right)$ may exist for $g<r-2$, and any pair $(r, g)$ describes an example when $g \geq r-2$.

Below are two pictures of the surface $\mathrm{DH}_{1,2}$, one showing it completely, the other exhibiting only the central planar end:
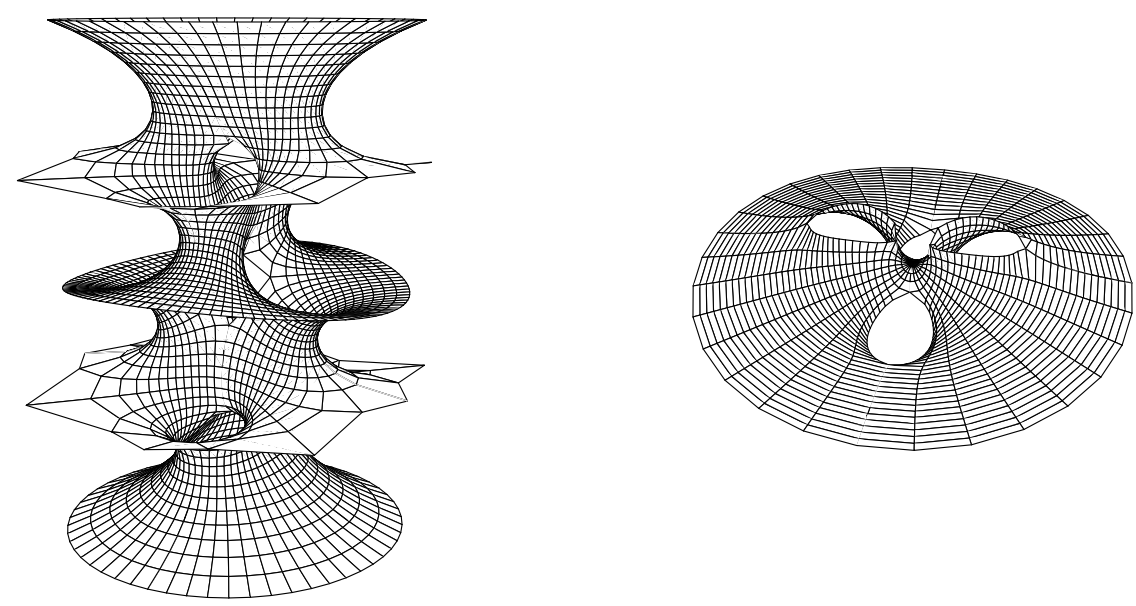

$\mathrm{DH}_{1,2}$ surface and middle end 
1.2. The proof. As one of our principal goals is the description of this method, we now give an overview; in $\S 2.3$, we give a fairly detailed sketch. In particular, our discussion in that subsection is quite general, as we aim to outline an approach to proving existence results for wide classes of minimal surfaces, even if we only carry out that plan for the specific classes described above.

Minimal surfaces in space can be defined in terms of their Weierstrass data: a pair of meromorphic forms on the underlying Riemann surface. Conversely, a pair of meromorphic forms on a simply connected domain naturally determines a (local) minimal surface, up to some mild compatibility requirement on the divisors. Defining a minimal surface with some homology is substantially more difficult however, as this requires a compatibility of periods of three one-forms (say $\left(\alpha_{1}, \alpha_{2}, \alpha_{3}\right)$ ) defined via the data. A common approach to this global "period problem" is to prescribe sufficient symmetry of the minimal surface so that the conformal structure of the minimal surface is apparent; one then searches for appropriate meromorphic one-forms $\alpha_{1}, \alpha_{2}, \alpha_{3}$ on that Riemann surface. As our minimal surfaces will have only a few symmetries, we are unable to determine the conformal structure of the surface a priori, or even to restrict it sufficiently well so that we might adapt that common approach; we develop a different approach. We handle this "period problem" by instead studying the (developed) flat structures associated to the $\alpha_{i}$ : in this formulation, the periods are identifiable as vectors in $\mathbb{C}$, and the period problem is soluble when those vectors are compatible (see $\S \S 3.2,3.3$ for the precise relationships). In fact, for many surfaces with interesting shapes, there are large moduli spaces of triples of flat structures whose geometries are compatible in the sense that if the underlying Riemann surfaces were conformally identical, the flat structures would correspond to Weierstrass data with a "solved" period problem.

Thus, we have translated the problem of producing a minimal surface in $\mathbb{E}^{3}$ with prescribed shape into a problem in Teichmüller theory: in a moduli space $\Delta$ of compatible triples of flat structures, find a triple whose underlying Riemann surfaces coincide. This we solve nonconstructively by introducing a nonnegative height function $\mathcal{H}: \Delta \rightarrow \mathbb{R}^{+}$on $\Delta$, which has the features of being proper, and whose only critical point is at a solution to our problem. The bulk of the paper is a description of this height function $(\S 4.3)$, a proof of its properness $(\S 4.6)$, and a proof that its only critical points are at solutions $(\S 5, \S 6)$. An interesting feature of this proof is that it is inductive: the triples of flat structures for a slightly less complicated minimal surface lie on a boundary face of the compactified moduli space $\bar{\Delta}$ of compatible triples of flat structures for more complicated surfaces. We consider this solution (on $\bar{\Delta}$ ) to the less complicated problem as the point-at-infinity of a particularly good locus in the moduli space $\Delta$ on which to restrict the height and look for a solution. 
This bootstrapping from slightly less complicated solutions to solutions is the 'handle addition' referred to in the title of the paper.

Along the way, we learn quite a bit about moduli spaces of pairs of flat structures. The bulk of $\S 4$ is devoted to describing asymptotic relationships between the underlying conformal structures of flat disks with alternating horizontal and vertical sides of corresponding lengths. Most of $\S 5$ concerns estimates on infinitesimal changes in the conformal structure of a flat structure given a prescribed infinitesimal change in its Euclidean geometry; $\S 6$ concerns the effects on a flat structure of the opening of a node in a Riemann surface.

Our pace in this exposition is occasionally casual, as we have tried to include a number of motivating examples along the way. Indeed, we feel these illustrate the power of our approach. For instance, it is occasionally immediately clear (and even a straightforward calculation) that the moduli space $\Delta$ for a shape is empty - we then conclude that there is no minimal surface with that shape (see $\S 3.5 .2, \S 7$ ). There are also instances when the moduli space is an identifiable singleton, from which we may conclude immediately that such a shape is realizable minimally; in $§ 3.5 .1$, we find that this is true for Costa's surface, obviating the need for the analysis of elliptic functions in the proof of the existence of this particular surface, and yielding a conceptually new proof of the existence of this surface.

Here is a detailed discussion of the contents of this paper: we begin in $\S 2$ with some background in Teichmüller theory and minimal surfaces, the two subjects which we relate in this paper. The proof of Theorem B occupies the bulk of the paper ( $\S 3$ through $\S 7, \S 9$ ). While the details are occasionally quite technical and the arguments require some space to present completely, the basic plan and ideas are rather straightforward; we give a step-by-step summary in $\S 2.3$. Our objects of study are minimal surfaces which have sufficient symmetries so that the fundamental domain for the action is a disk. An equivariant form on that surface induces a flat structure on the disk with straight boundaries, and we study such domains ("orthodisks") and their moduli spaces in $\S 3$. Also in $\S 3$, we meet our main new technical obstacle: we allow our domains to develop into $\mathbb{E}^{2}$ as branched covers of the plane. This introduces many complications into the analysis in terms of allowing many different types of geometrically defined motions and degenerations within the moduli spaces, as well as some difficulties in defining the frontiers of the moduli space. We deal with these difficulties in $\S 4$, where we introduce our height function and prove its properness. In $\S 6$ we find a good locus within moduli space on which to flow to a solution, and in $\S 5$ we prove that we may flow along that locus to a solution. In $\S 7$, we prove the nonexistence portion of the classification Theorem B, and we conclude in $\S 8$ by extending some of our results: we extend to cases of higher dihedral symmetry in $\S 8.1$, and to nonplanar ends in $\S 8.2$. 
Finally, in $\S 8.3$, we end by offering evidence that our surfaces are embedded; in particular, we state that the surfaces are regularly homotopic to an embedding, by a compactly supported regular homotopy. (Space considerations force us to defer the proofs of the statements in $\S 8.2$ and $\S 8.3$ to the book [WW2].)

Acknowledgments. It is once again a pleasure to acknowledge our indebtedness to Hermann Karcher for many conversations related to this work, and for a very careful reading which led to a much improved exposition. We also appreciate the helpful comments of the referee.

\section{Background, notation, and a sketch of the argument}

\subsection{Minimal surfaces.}

2.1.1. The Weierstrass representation. Any complete minimal surface $M$ of finite total curvature in $\mathbb{E}^{3}$ can be defined by

$$
z \mapsto \operatorname{Re} \int^{z}\left(\omega_{1}, \omega_{2}, \omega_{3}\right)
$$

where $\omega_{i}$ are three meromorphic 1 -forms on a compact Riemann surface $\mathcal{R}$ such that

$$
\omega_{1}^{2}+\omega_{2}^{2}+\omega_{3}^{2} \equiv 0
$$

This last condition is usually eliminated by writing

$$
\omega_{1}=\frac{1}{2}\left(G-G^{-1}\right) d h, \omega_{2}=\frac{i}{2}\left(G+G^{-1}\right) d h, \omega_{3}=d h
$$

where $G$ is the Gauss map and $d h$ the height differential. Here $d h$ is a closed (but not necessarily exact) differential on the underlying Riemann surface $\mathcal{R}$.

The pair $G$ and $d h$ are called the Weierstrass data for the minimal surface $M$.

Significant geometric data attached to such a surface are the total absolute curvature

$$
\mathcal{K}:=\int_{R}|K| d A=4 \pi \cdot \text { degree of the Gauss map }
$$

and its Riemannian metric

$$
d s=\left(|G|+\frac{1}{|G|}\right)|d h| .
$$

At an end, the value of the Gauss map gives the normal to the asymptotic plane. The metric becomes infinite by completeness, and the order of decay or degeneration describes the type of end: The only possible embedded ends are Catenoid ends and planar ends for which

$$
d s \sim \infty^{2} .
$$


They are distinguished by the Gauss map: for a catenoid end, it is single-valued and for a flat end, the Gauss map has higher multiplicity. For the planar ends we will construct, we will assume that the Gauss map at a flat end has local degree three.

Given a Riemann surface $\mathcal{R}$, a meromorphic function $G$ on $\mathcal{R}$ and a meromorphic form $d h$ on $\mathcal{R}$ for which the metric $d s$ above is regular except at an acceptable collection of distinguished points on $\mathcal{R}$, we can attempt to use the constructed forms $\omega_{i}$ and the formula (called the Weierstrass representation)

$$
z \mapsto \operatorname{Re} \int_{.}^{z}\left(\frac{1}{2}\left(G-G^{-1}\right) d h, \frac{i}{2}\left(G+G^{-1}\right) d h, d h\right)
$$

to define a minimal surface in space. This procedure works locally, but the surface is only well-defined globally if the periods

$$
\operatorname{Re} \int_{\gamma}\left(\frac{1}{2}\left(G-G^{-1}\right) d h, \frac{i}{2}\left(G+G^{-1}\right) d h, d h\right)
$$

vanish for every cycle $\gamma \subset \mathcal{R}$. The problem of finding compatible meromorphic data $(G, d h)$ which satisfy the above conditions on the periods of $\omega_{i}$ is known as 'the period problem for the Weierstrass representation'.

We will find it convenient to use the following psychologically different well-known version of the above period conditions and Weierstrass data: we will attempt to specify compatible forms $G d h$ and $G^{-1} d h$ so that the periods satisfy

$$
\operatorname{Re} \int_{\gamma} d h=0
$$

and

$$
\int_{\gamma} G d h=\overline{\int_{\gamma} G^{-1} d h .}
$$

The equivalence to the original period problem is elementary.

The forms $\alpha_{1}=G d h, \alpha_{2}=G^{-1} d h$, and $\alpha_{3}=d h$ lead to singular flat structures on the underlying Riemann surfaces, defined via the line elements $d s_{\alpha_{i}}=\left|\alpha_{i}\right|$. These singular metrics are flat away from the support of the divisor of $\alpha_{i}$; on elements $p$ of that divisor, the metrics have cone points with angles equal to $2 \pi\left(\operatorname{ord}_{\alpha_{i}}(p)+1\right)$. More importantly, the periods of the forms are given by the Euclidean geometry of the developed image of the metric $d s_{\alpha_{i}}$ - a period of a cycle $\gamma$ is the distance in $\mathbb{C}$ between consecutive images of a distinguished point in $\gamma$. We reverse this procedure in $\S 3$ : we use putative developed images of the one-forms $G d h, G^{-1} d h$, and $d h$ to solve formally the period problem for some formal Weierstrass data. 
2.2. Teichmüller theory. For $M$ a smooth surface, let Teich $(M)$ denote the Teichmüller space of all conformal structures on $M$ under the equivalence relation given by pullback by diffeomorphisms isotopic to the identity map id: $M \longrightarrow M$. Then it is well-known that Teich $(M)$ is a smooth finite dimensional manifold if $M$ is a closed surface.

There are two spaces of tensors on a Riemann surface $\mathcal{R}$ that are important for the Teichmüller theory. The first is the space $\mathrm{QD}(\mathcal{R})$ of holomorphic quadratic differentials, i.e., tensors which have the local form $\Phi=\varphi(z) d z^{2}$ where $\varphi(z)$ is holomorphic. The second is the space of Beltrami differentials $\operatorname{Belt}(\mathcal{R})$, i.e., tensors which have the local form $\mu=\mu(z) d \bar{z} / d z$.

The cotangent space $T_{[\mathcal{R}]}^{*}($ Teich $(M))$ is canonically isomorphic to $\mathrm{QD}(\mathcal{R})$, and the tangent space is given by equivalence classes of (infinitesimal) Beltrami differentials, where $\mu_{1}$ is equivalent to $\mu_{2}$ if

$$
\int_{\mathcal{R}} \Phi\left(\mu_{1}-\mu_{2}\right)=0 \quad \text { for every } \Phi \in \mathrm{QD}(\mathcal{R}) .
$$

If $f: \mathbb{C} \rightarrow \mathbb{C}$ is a diffeomorphism, then the Beltrami differential associated to the pullback conformal structure is $\nu=\frac{f_{\bar{z}}}{f_{z}} \frac{d \bar{z}}{d z}$. If $f_{\varepsilon}$ is a family of such diffeomorphisms with $f_{0}=\mathrm{id}$, then the infinitesimal Beltrami differential is given by $\left.\frac{d}{d \varepsilon}\right|_{\varepsilon=0} \nu_{f_{\varepsilon}}=\left(\left.\frac{d}{d \varepsilon}\right|_{\varepsilon=0} f_{\varepsilon}\right)_{\bar{z}}$. We will carry out an example of this computation in $\S 5.2$.

A holomorphic quadratic differential comes with a picture that is a useful aid to one's intuition about them. The picture is that of a pair of transverse measured foliations, whose properties we sketch briefly (see [FLP] for more details).

A $C^{k}$ measured foliation on $\mathcal{R}$ with singularities $z_{1}, \ldots, z_{l}$ of order $k_{1}, \ldots, k_{l}$ (respectively) is given by an open covering $\left\{U_{i}\right\}$ of $\mathcal{R}-\left\{z_{1}, \ldots, z_{l}\right\}$ and open sets $V_{1}, \ldots, V_{l}$ around $z_{1}, \ldots, z_{l}$ (respectively) along with real valued $C^{k}$ functions $v_{i}$ defined on $U_{i}$ such that

(i) $\left|d v_{i}\right|=\left|d v_{j}\right|$ on $U_{i} \cap U_{j}$,

(ii) $\left|d v_{i}\right|=\left|\operatorname{Im}\left(z-z_{j}\right)^{k_{j} / 2} d z\right|$ on $U_{i} \cap V_{j} i$.

Evidently, the kernels ker $d v_{i}$ define a $C^{k-1}$ line field on $\mathcal{R}$ which integrates to give a foliation $\mathcal{F}$ on $\mathcal{R}-\left\{z_{1}, \ldots, z_{l}\right\}$, with a $k_{j}+2$-pronged singularity at $z_{j}$. Moreover, given an $\operatorname{arc} A \subset \mathcal{R}$, we have a well-defined measure $\mu(A)$ given by

$$
\mu(A)=\int_{A}|d v|
$$

where $|d v|$ is defined by $|d v|_{U_{i}}=\left|d v_{i}\right|$. An important feature required of this measure is its "translation invariance". That is, suppose $A_{0} \subset \mathcal{R}$ is an arc transverse to the foliation $\mathcal{F}$, with $\partial A_{0}$ a pair of points, one on the leaf $l$ and one on the leaf $l^{\prime}$; then, if we deform $A_{0}$ to $A_{1}$ via an isotopy through $\operatorname{arcs} A_{t}$ 
that maintains the transversality of the image of $A_{0}$ at every time, and also keeps the endpoints of the $\operatorname{arcs} A_{t}$ fixed on the leaves $l$ and $l^{\prime}$, respectively, then we require that $\mu\left(A_{0}\right)=\mu\left(A_{1}\right)$.

Now a holomorphic quadratic differential $\Phi$ defines a measured foliation in the following way. The zeros $\Phi^{-1}(0)$ of $\Phi$ are well-defined; away from these zeros, we can choose a canonical conformal coordinate $\zeta(z)=\int^{z} \sqrt{\Phi}$ so that $\Phi=d \zeta^{2}$. The local measured foliations $(\{\operatorname{Re} \zeta=$ const $\},|d \operatorname{Re} \zeta|)$ then piece together to form a measured foliation known as the vertical measured foliation of $\Phi$, with the translation invariance of this measured foliation of $\Phi$ following from Cauchy's theorem.

Work of Hubbard and Masur ([HM]) (see also alternate proofs in [Ke], $[\mathrm{Gar}]$ and $[\mathrm{Wo}]$ ), following Jenkins ([J]) and Strebel ([Str]), showed that given a measured foliation $(\mathcal{F}, \mu)$ and a Riemann surface $\mathcal{R}$, there is a unique holomorphic quadratic differential $\Phi_{\mu}$ on $\mathcal{R}$ so that the horizontal measured foliation of $\Phi_{\mu}$ is equivalent to $(\mathcal{F}, \mu)$.

Extremal length. The extremal length $\operatorname{Ext}_{\mathcal{R}}([\gamma])$ of a class of $\operatorname{arcs} \Gamma$ on a Riemann surface $\mathcal{R}$ is defined to be the conformal invariant

$$
\operatorname{Ext}_{\mathcal{R}}([\gamma])=\sup _{\rho} \frac{\ell_{\rho}^{2}(\Gamma)}{\operatorname{Area}(\rho)}
$$

where $\rho$ ranges over all conformal metrics on $\mathcal{R}$ with areas $0<\operatorname{Area}(\rho)<\infty$ and $\ell_{\rho}(\Gamma)$ denotes the infimum of $\rho$-lengths of curves $\gamma \in \Gamma$. Here $\Gamma$ may consist of all curves freely homotopic to a given curve, a union of free homotopy classes, a family of arcs with endpoints in a pair of given boundaries, or even a more general class. Kerckhoff ([Ke]) showed that this definition of extremal lengths of curves extended naturally to a definition of extremal lengths of measured foliations.

For a class $\Gamma$ consisting of all curves freely homotopic to a single curve $\gamma \subset M$, (or more generally, a measured foliation $(\mathcal{F}, \mu)$ ) we see that $\operatorname{Ext}_{(\cdot)}(\Gamma)$

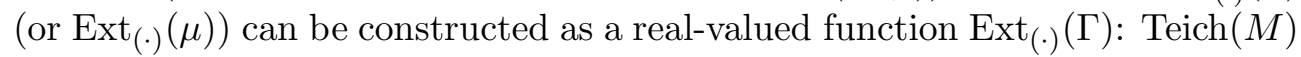
$\longrightarrow \mathbb{R}$. Gardiner $([\mathrm{Gar}])$ showed that $\operatorname{Ext}_{(\cdot)}(\mu)$ is differentiable and Gardiner and Masur $([\mathrm{GM}])$ showed that $\operatorname{Ext}_{(\cdot)}(\mu) \in C^{1}$ (Teich $\left.(M)\right)$. (In our particular applications, the extremal length functions on our moduli spaces will be real analytic; this will be explained in $§ 4.5$.) Moreover Gardiner computed that

$$
\left.d \operatorname{Ext}_{(\cdot)}(\mu)\right|_{[\mathcal{R}]}=2 \Phi_{\mu}
$$

so that

$$
\left(\left.d \operatorname{Ext}_{(\cdot)}(\mu)\right|_{[\mathcal{R}]}\right)[\nu]=4 \operatorname{Re} \int_{\mathcal{R}} \Phi_{\mu} \nu .
$$

This formula will be crucial to our discussion in $§ 5.3$. 
2.3. A brief sketch of the proof. While the details of the arguments are sometimes quite involved, the basic logic of the approach and the ideas of the proofs are quite simple. Moreover, it is quite likely that they extend to large classes of other minimal surfaces (see [MW], [WW2]), so in this subsection, we sketch the approach, as a step-by-step recipe.

Step 1. Draw the surface. The first step in proving the existence of a minimal surface is to work out a detailed proposal. This can either be done numerically, as in the work of Thayer ([Th]) for the Chen-Gackstatter surfaces and Boix and Wohlgemuth ([Bo], [Woh2]) for the low genus surfaces we treat here, or it can be schematic, showing how various portions of the surface might fit together. We follow the latter approach here, which requires quite a bit of terminology - there are many ways to orient and order the handles and ends in a surface of genus five with five planar ends and two catenoid ends, even under an additional restriction on symmetry. To narrow the list of possibilities, one applies either some numerical work, or some intuition, or one attempts to continue with the outline of the proof. We develop the terminology for this process and define the models for some of our candidates in $\S 3.6$, and for the rest of them in $\S 3.7$.

Step 2. Compute the divisors for the forms $G d h$ and $G^{-1} d h$. From the model that we drew in Step 1, we can compute the divisors for the Weierstrass data, which we just defined to be the Gauss map $G$ and the 'height' form $d h$. (Note here how important it is that the Weierstrass representation be given in terms of geometrically defined quantities - for us, this gives the passage between the extrinsic geometry of the minimal surface as defined in Step 1 and the conformal geometry and Teichmüller theory of the later steps.) Thus we can also compute the divisors for the meromorphic forms $G d h$ and $G^{-1} d h$ on the Riemann surface (so far undetermined, but assumed to exist) underlying the minimal surface. Of course the divisors for a form determine the form up to a constant, so the divisor information nearly determines the Weierstrass data for our surface. These Weierstrass data are computed in $\S 3.6$ (and $\S 3.7$, for an extended class) as well.

Step 3. Compute the flat structures for the forms $G d h$ and $G^{-1} d h$ required by the period conditions. A meromorphic form on a Riemann surface defines a flat singular (conformal) metric on that surface; for example, from the form $G d h$ on our putative Riemann surface, we determine a line element $d s_{G d h}=$ $|G d h|$. This metric is locally Euclidean away from the support of the divisor of the form and has a complete Euclidean cone structure in a neighborhood of a zero or pole of the form. Thus we can develop the universal cover of the surface into the Euclidean plane. 
The flat structures for the forms $G d h$ and $G^{-1} d h$ are not completely arbitrary; because the periods for the pair of forms must be conjugate (formula (2.1b)), the flat structures must develop into domains which have a particular Euclidean geometric relationship to one another. This relationship is crucial to our approach, so we will dwell on it somewhat. If the map $D: \Omega \longrightarrow \mathbb{E}^{2}$ is the map which develops the flat structure of a form, say $\alpha$, on a domain $\Omega$ into $\mathbb{E}^{2}$, then the map $D$ pulls back the canonical form $d z$ on $\mathbb{C} \cong \mathbb{E}^{2}$ to the form $\alpha$ on $\Omega$. Thus the periods of $\alpha$ on the Riemann surface are given by integrals of $d z$ along the developed image of paths in $\mathbb{C}$, i.e. by differences of the complex numbers representing endpoints of those paths in $\mathbb{C}$.

We construe all of this as requiring that the flat structures develop into domains that are "conjugate": if we collect all of the differences in positions of parallel sides for the developed image of the form $G d h$ into a large complexvalued $n$-tuple $V_{G d h}$, and we collect all of the differences in positions of corresponding parallel sides for the developed image of the form $G^{-1} d h$ into a large complex-valued n-tuple $V_{G^{-1} d h}$, then these two complex-valued vectors $V_{G d h}$ and $V_{G^{-1} d h}$ should be conjugate. This is the flat structure implication of the period condition formula $(2.1 \mathrm{~b})$, here using that our situation allows that the periods of all cycles can be found from differences of positions of parallel sides in a flat structure. Thus, we translate the "period problem" into a statement about the Euclidean geometry of the developed flat structures. This is done at the end of $\S 3.6$ (and again in $\S 3.7$, for the extended class of surfaces).

The period problem (2.1a) for the form $d h$ will be trivially solved for the surfaces we treat here.

Step 4. Define the moduli space of pairs of conjugate flat domains. Now we work backwards. We know the general form of the developed images (called $\Omega_{G d h}$ and $\Omega_{G^{-1} d h}$, respectively) of flat structures associated to the forms $G d h$ and $G^{-1} d h$, but in general, there are quite a few parameters of the flat structures left undetermined, even after we have assumed symmetries, determined the Weierstrass divisor data for the models and used the period conditions (2.1b) to restrict the relative Euclidean geometries of the pair $\Omega_{G d h}$ and $\Omega_{G^{-1}} d h$. Thus, there is a moduli space $\Delta$ of possible candidates of pairs $\Omega_{G d h}$ and $\Omega_{G^{-1} d h}$; our period problem (condition $(2.1 \mathrm{~b})$ ) is now a conformal problem of finding such pairs which are conformally equivalent by a map which preserves the corresponding cone points. (Solving this problem means that there is a well-defined Riemann surface which can be developed into $\mathbb{E}^{2}$ in two ways, so that the pair of pullbacks of the form $d z$ gives forms $G d h$ and $G^{-1} d h$ with conjugate periods.)

The condition of conjugacy of the domains $\Omega_{G d h}$ and $\Omega_{G^{-1} d h}$ often dictates some restrictions on the moduli space, and even a collection of geometrically defined coordinates. We work these out in the $\S 4.2$ and $\S 4.4$. 
When these moduli spaces are empty, we have a proof of nonexistence: this is the case for the surfaces studied in part (ii) of Theorem B - see $\S 7$.

Step 5. Solve the conformal problem using Teichmúller theory. At this juncture, our minimal surface problem has become a problem in finding a special point in a product of moduli spaces of complex domains; we will have no further references to minimal surface theory. The plan is straightforward: we will define a height function $\mathcal{H}: \Delta \longrightarrow \mathbb{R}$ with the properties:

1 (Reflexivity) The height $\mathcal{H}$ equals 0 only at a solution to the conformal problem.

2 (Properness) The height $\mathcal{H}$ is proper on $\Delta$. This ensures the existence of a critical point.

3 (Noncritical Flow) If the height $\mathcal{H}$ at a pair $\left(\Omega_{G d h}, \Omega_{G^{-1} d h}\right)$ does not vanish, then the height $\mathcal{H}$ is not critical at that pair.

This is clearly enough to solve the problem: we now sketch the proofs of these steps.

Step 5a. Reflexivity. We need conformal invariants of a domain that provide a complete set of invariants (for Reflexivity), have estimable asymptotics (for Properness), and computable first derivatives in moduli space (for the Noncritical Flow Property). One obvious choice is a set of functions of extremal lengths for a good choice of curve systems, say $\Gamma=\left\{\gamma_{1}, \ldots, \gamma_{K}\right\}$ on the domains; it is sufficient for Reflexivity that the extremal lengths of these curves provide coordinates for $\Delta$. Suitable curve systems are defined for our examples in $\S 4.2$ and $\S 4.4$. We then define a height function $\mathcal{H}$ which vanishes only when there is agreement between all of the extremal lengths $\operatorname{Ext}_{\Omega_{G d h}}\left(\gamma_{i}\right)=$ $\operatorname{Ext}_{\Omega_{G^{-1}} d h}\left(\gamma_{i}\right)$ and which blows up when $\operatorname{Ext}_{\Omega_{G d h}}\left(\gamma_{i}\right)$ and $\operatorname{Ext}_{\Omega_{G^{-1}}{ }_{d h}}\left(\gamma_{i}\right)$ either decay or blow up at different rates. See for example Definition 4.3.1 and Lemma 4.5.5.

Step 5b. Properness. Our height function will measure differences in the extremal lengths $\operatorname{Ext}_{\Omega_{G d h}}\left(\gamma_{i}\right)$ and $\operatorname{Ext}_{\Omega_{G^{-1}} d h}\left(\gamma_{i}\right)$. Often, but not always, a geometric degeneration of the flat structure of either $\Omega_{G d h}$ or $\Omega_{G^{-1} d h}$ will force one of the extremal lengths Ext. $\left(\gamma_{i}\right)$ to tend to zero or infinity, while the other extremal length stays finite and bounded away from zero. This is a straightforward situation where it will be obvious that the height function will blow up. A more subtle case arises when a geometric degeneration of the flat structure forces both of the extremal lengths $\operatorname{Ext}_{\Omega_{G d h}}\left(\gamma_{i}\right)$ and $\operatorname{Ext}_{\Omega_{G^{-1}} h}\left(\gamma_{i}\right)$ to decay simultaneously (or explode). In that case, we begin by observing that there is a natural map between the vector $\left\langle\operatorname{Ext}_{\Omega_{G d h}}\left(\gamma_{i}\right)\right\rangle$ and the vector $\left\langle\operatorname{Ext}_{\Omega_{G^{-1}} d h}\left(\gamma_{i}\right)\right\rangle$. This pair of vectors is reminiscent of pairs of solutions to a 
hypergeometric differential equation, and we show, by a monodromy argument analogous to that used in the study of those equations, that it is not possible for corresponding components of that vector to vanish or blow up at identical rates. In particular, we show that the logarithmic terms in the asymptotic expansion of the extremal lengths near zero have a different sign, and this sign difference forces a difference in the rates of decay that is detected by the height function, forcing it to blow up in this case. The monodromy argument is given in $\S 4.7$, and the properness discussion consumes $\S 4$.

Step 5c. Noncritical flow. The domains $\Omega_{G d h}$ and $\Omega_{G^{-1} d h}$ have a remarkable property: if $\operatorname{Ext}_{\Omega_{G d h}}\left(\gamma_{i}\right)>\operatorname{Ext}_{\Omega_{G^{-1}}\left(\gamma_{i}\right.}\left(\gamma_{i}\right)$, then there are always deformations so that when we deform $\Omega_{G d h}$ so as to decrease $\operatorname{Ext}_{\Omega_{G d h}}\left(\gamma_{i}\right)$, the conjugacy condition forces us to deform $\Omega_{G^{-1} d h}$ so as to increase $\operatorname{Ext}_{\Omega_{G^{-1}} d h}\left(\gamma_{i}\right)$. We can thus always deform $\Omega_{G d h}$ and $\Omega_{G^{-1} d h}$ in order to reduce one term of the height function $\mathcal{H}$. We develop this step in $\S 5$.

Step 5d. Regeneration. In the process described in the previous step, an issue arises: we might be able to reduce one term of the height function via a deformation, but this might affect the other terms, so as not to provide an overall decrease in height. We thus seek a locus $\mathcal{Y}$ in our moduli space where the height function has but a single nonvanishing term, and all the other terms vanish to at least second order. If we can find such a locus $\mathcal{Y}$, we can flow along that locus to a solution. To begin our search for such a locus, we observe which flat domains arise as limits of our domains $\Omega_{G d h}$ and $\Omega_{G^{-1} d h}$ : commonly, the degenerate domains are the flat domains for a similar minimal surface problem, maybe of slightly lower genus or a few fewer ends.

We find our desired locus by considering the boundary $\partial \bar{\Delta}$ of the closure $\bar{\Delta}$ of the moduli space $\Delta$; this boundary has strata of moduli spaces $\Delta^{\prime}$ for minimal surface problems of lower complexity. By induction, there is a solution $X^{\prime}$ of those problems represented on such a boundary strata $\Delta^{\prime}$ (with all of the corresponding extremal lengths in agreement), and we prove that there is a locus $\mathcal{Y} \subset \Delta$ inside the larger moduli space $\Delta$ (with $\mathcal{Y}$ limiting on $X^{\prime}$ ) which has the analogues of those same extremal lengths in agreement. As a corollary of that condition, the height function on $\mathcal{Y}$ has the desired simple properties. This is developed in $\S 6$.

The proof may be summarized as follows: we restrict to a locus $\mathcal{Y}$ on which we have the Noncritical Flow Property. By step 5b, the height function $\mathcal{H}$ is proper on $\mathcal{Y}$; thus there is a critical point $X$ on $\mathcal{Y}$ for $\mathcal{H}$. The Noncritical Flow then forces $\mathcal{H}(X)=0$, so by Reflexivity, the surface represented by $X$ is a solution to our conformal problem, and hence also defines a solution to the minimal surface problem. 


\section{The geometry of orthodisks}

In this section we introduce the notion of orthodisks.

3.1. Orthodisks. Consider the upper half-plane and $n \geq 3$ distinguished points $t_{i}$ on the real line. The point $t_{\infty}=\infty$ will also be a distinguished point. We will refer to the upper half-plane together with these data as a conformal polygon and to the distinguished points as vertices. Two conformal polygons are conformally equivalent if there is a biholomorphic map between the disks carrying vertices to vertices, and fixing $\infty$.

Let $a_{i}$ be some odd integers such that

$$
a_{\infty}=-4-\sum_{i} a_{i}
$$

By a Schwarz-Christoffel map we mean the map

$$
F: z \mapsto \int_{i}^{z}\left(t-t_{1}\right)^{a_{1} / 2} \cdot \ldots \cdot\left(t-t_{n}\right)^{a_{n} / 2} d t
$$

A point $t_{i}$ with $a_{i}>-2$ is called finite, otherwise infinite. By (3.1), there is at least one finite vertex (possibly at $t_{\infty}$ ).

Definition 3.1.1. Let $a_{i}$ be odd integers. The pull-back of the flat metric on $\mathbb{C}$ by $F$ defines a complete flat metric with boundary on $\mathbb{H} \cup \mathbb{R}$ without the infinite vertices. We call such a metric an orthodisk. The $a_{i}$ are called the vertex data of the orthodisk. The edges of an orthodisk are the boundary segments between vertices; they come in a natural order. Consecutive edges meet orthogonally at the finite vertices. Every other edge is parallel under the parallelism induced by the flat metric of the orthodisk. Oriented distances between parallel edges are called periods. The periods can have four different signs: $+1,-1,+i,-i$.

Remark. The integer $a_{i}$ corresponds to an angle $\left(a_{i}+2\right) \pi / 2$ of the orthodisk. Negative angles are meaningful because a vertex (with a negative angle $-\theta$ ) lies at infinity and is the intersection of a pair of lines which also intersect at a finite point, where they make a positive angle of $+\theta$.

In all the drawings of the orthodisks to follow, we mean the domain to be to the left of the boundary.

Example 3.1.2. This is conceivably the simplest orthodisk, bounding the second quadrant in $\mathbb{R}^{2}$ : 


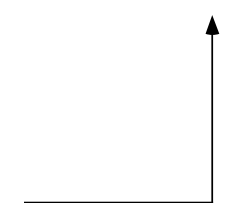

Simple orthodisk

Example 3.1.3. Here is an orthodisk with a branch point which is drawn fat. The disk consists of the region northwest of the larger 5-vertex boundary and the region northwest of the fat vertex boundary.

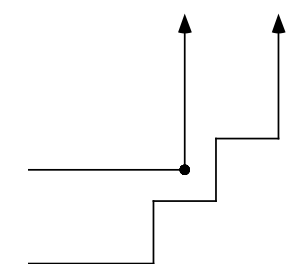

Branched orthodisk ...

To get a clear picture of this orthodisk, we can glue the following two domains together along the fat diagonal boundary line, in exactly the way that a Riemann surface is assembled from several sheets and a branch cut.
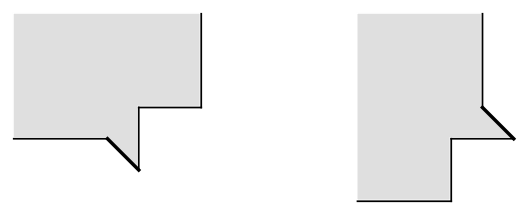

... decomposed into two pieces

Denote by $\gamma_{i}$ an oriented curve connecting edge $t_{i} t_{i+1}$ with edge $t_{i+2} t_{i+3}$. There are $n-1$ such curves. We will denote by the same name, also, their homotopy classes. It is well-known that the extremal lengths $\operatorname{Ext}\left(\gamma_{i}\right)$ determine the conformal structure of the conformal polygon. This follows because the extremal lengths of a conformal quadrilateral determine the cross-ratios of the vertices of the quadrilateral, and the cross-ratios then determine the vertices of the polygon, where we view the vertices as distinguished points on the boundary of, say, the disk.

Each orthodisk has a natural conformal structure and hence determines a conformal polygon. Vice versa, given a conformal polygon and applying the Schwarz-Christoffel map to it, one obtains an orthodisk with certain periods. 
By scaling, one can arrange for one period to be (say) 1. We then call this map a normalized period map.

Applying the Schwarz-Christoffel discussion above, and then using the implicit function theorem, one can prove the following proposition (which we will never use, so we omit a detailed proof):

LEMma 3.1.4. The normalized map from the space of conformal polygons to periods of orthodisks of given vertex data is locally injective.

We will restrict our attention to a subclass of orthodisks which have a real symmetry.

Definition 3.1.5. An orthodisk is called symmetric if it has a reflectional symmetry which fixes two vertices.

All of the orthodisks under discussion have angles which are odd multiples of $\pi / 2$, and, with but two exceptional cases of the vertices representing the catenoid ends, the angles alternate between being congruent to $\pi / 2$ and congruent to $3 \pi / 2$ modulo $2 \pi$; the result of this combinatorics of angles is that any symmetric orthodisk must fix a pair of vertices that are on 'opposite' sides of the orthodisks, each halfway between the vertices representing the catenoid ends.

The above Examples 3.1.2 and 3.1.3 are symmetric. As a convention we will draw all orthodisks such that the symmetry line is the diagonal $y=-x$.

3.2. From orthodisks to Riemann surfaces. We begin with an orthodisk $X$ and we describe in this section a method to canonically construct a hyperelliptic Riemann surface $Z$ with a meromorphic 1-form $\omega_{X}$ from $\mathrm{X}$.

Let $X$ be a conformal polygon. First double $X$ along its boundary; i.e., take $X$ and a copy $\bar{X}$ (with the opposite orientation) and glue $X$ and $\bar{X}$ together along their commonly labelled edges. The resulting complex space $Y$ is then topologically a sphere with distinguished points which we also call $t_{i}$; moreover, the sphere $Y$ (punctured at the infinite vertices) inherits a complete singular flat structure from $X$ and $\bar{X}$. To see this last statement, begin by observing that the flat structure on $(\operatorname{int} X) \cup \operatorname{int}(\bar{X})$ extends across the interior of the boundary segments of $\partial X$ and $\partial \bar{X}$ because those segments are straight. At the finite vertices, the flat structure has cone-like singularities, while the infinite vertices are at infinite distance from any point in a neighborhood of them. More precisely, we compute that at the finite vertices, the cone angle at the vertex of the sphere $Y$ corresponding to $t_{i}$ will be $\alpha_{i}=2 \pi\left(a_{i} / 2+1\right)$. We use formally the same formula to attach (negative) cone angles to the vertices at infinity. In fact the end corresponding to a vertex $t_{i}$ at infinity in $\mathbb{C}$ is conformally the same as the infinite end of a cone with cone angle $-\alpha_{i}$. 
Next, construct simple closed curves $c_{i}=\gamma_{i} \cup \overline{\gamma_{i}}$ on $Y$ by connecting $\gamma_{i}$ on $X$ with the oppositely oriented $\operatorname{arc} \overline{\gamma_{i}}$ on $\bar{X}$. Now, as $Y$ is a double of $X$, the extremal lengths of the $c_{i}$ determine the conformal structure of the punctured sphere $Y$. Finally, construct the hyperelliptic double cover $Z$ over $Y$ branched over the vertices $t_{i}$. The conformal structure of this covering is independent of the choice of branching slits. However, this is not true for its Teichmüller class: the choice of slits affects the marking of the surface, i.e., the choice of a basis for $\pi_{1} Z$. Because we soon want to measure distances in Teichmüller space between points constructed this way, we need to choose the slits consistently: we take as branch slits the odd numbered edges. It is then easy to check that $c_{i}$ has two lifts to $Z$ whose sum is null homologous. To pick a specific lift, we make, once and for all, a choice: choose, for each $i$, a lift $\tilde{c}_{i}$ of $c_{i}$. Subsequent constructions will depend on that choice, so we will have to ensure that the final statements are independent of this choice.

Metrically, on the double cover $Z$ we obtain a lifted flat cone metric with cone angles $\tilde{\alpha}_{i}=2 \pi\left(a_{i}+2\right)$ at the lifts of $t_{i}$ : this angle is an odd multiple of $2 \pi$. Furthermore, because only parallel edges of $X$ and $\bar{X}$ are glued together in the whole construction, the cone metric on $Z$ has no linear holonomy. Hence the exterior derivative of the (locally defined) developing map of the flat structure defines a global holomorphic 1-form $\omega_{X}$ on $Z$. (In slightly different language: we develop the flat structure on $Z$ into the Euclidean plane $\mathbb{E}^{2}$, on which there is defined the one form $d z$, in the usual coordinates. Because the flat structures on the orthodisks $X$ and $\bar{X}$ are bounded by horizontal and vertical lines, and our identifications take horizontal edges to horizontal edges and vertical edges to vertical edges, the transition maps between the distinct developed images of, say, $X$, are given by translations. Thus the form $d z$ pulls back to a welldefined one-form $\omega_{X}$ on $Z$.) This form $\omega_{X}$ has zeroes at the lifts of $t_{i}$ of order $a_{i}+1$; here, negative orders correspond to poles. Because the developing map is only defined up to a complex linear transformation, the 1-form $\omega_{X}$ will only be defined up to homothety.

Lemma 3.2.1. Up to a factor independent of $i$, the period of $\omega_{X}$ along $\tilde{c}_{i}$ is the period of $\gamma_{i}$.

Proof. Note that the developing map of a flat metric is (locally) only well-defined up to post-composition with a complex linear transformation, so that the 1-form $\omega$ is well-defined up to multiplication by some nonvanishing complex number. After having made that choice, a period of $\omega$ along $\tilde{c}_{i}$ is by construction the difference between the image (under the developing map defining this choice of $\omega$ ) of the endpoint of $c_{i}$ and the image of its initial point; this is because integrals of $\omega_{X}$ along paths in $Z$ push down via the developing map to integrals of $d z$ along pushed-forward paths in $\mathbb{E}^{2} \cong \mathbb{C}$. 
3.3. From orthodisks to Weierstrass data. We describe how pairs of orthodisks can be used to write down formal Weierstrass data for minimal surfaces. This procedure reverses the construction of $\S 2.1 .1$ where we find the flat structures associated to a minimal surface; here we will begin to solve the period problem for a minimal surface by first specifying pairs of relevant flat structures (formally $\Omega_{G d h}$ and $\Omega_{G^{-1} d h}$ ) whose geometry represents compatible periods, in the sense of $\S 2.1 .1$.

Definition 3.3.1. Two orthodisks $X_{1}$ and $X_{2}$ on the same underlying conformal polygon but with different angles (exponents) are called conformal. By the construction above, they give rise to two distinct meromorphic 1-forms $\omega_{1}, \omega_{2}$ on the same Riemann surface $Z$.

Definition 3.3.2. Two orthodisks $X_{1}$ and $X_{2}$ with different vertex data are called conjugate if there is a line $l \subset \mathbb{C}$ so that corresponding periods are symmetric with respect to that line.

Definition 3.3.3. Two orthodisks $X_{1}$ and $X_{2}$ are called reflexive if they are conformal and conjugate.

Now suppose that the pair of orthodisks $X_{1}$ and $X_{2}$ are defined on the same conformal polygon. Let the corresponding 1-forms on $Z$ be denoted $\omega_{1}$ and $\omega_{2}$. We want to find a meromorphic function $G$ (the Gauss map) and a meromorphic 1-form $d h$ (the height differential) such that

$$
\begin{aligned}
& \omega_{1}=G d h, \\
& \omega_{2}=G^{-1} d h
\end{aligned}
$$

and such that $G$ and $d h$ are Weierstrass data of a minimal surface on $Z$.

To solve the above equations, denote the vertex data of $X_{1}$ by $a_{i}$ and the vertex data of $X_{2}$ by $b_{i}$ (recall that these are odd numbers). Then at a point $t_{i}$ on the putative underlying Riemann surface, the meromorphic quadratic differential $\omega_{1} \omega_{2}$ has a zero of order $a_{i}+b_{i}+2$ which is an even number; thus, we first ask for $d h$ with zero of order $\frac{1}{2}\left(a_{i}+b_{i}\right)+1$.

Here is a simply defined but quite general case where one can find such a $d h$ :

Lemma 3.3.4. Suppose that, for each index $i$, the sum $a_{i}+b_{i} \equiv 0(\bmod 2)$. Then there is a meromorphic 1-form $d h$ on $Z$ as above with all periods purely imaginary.

Proof. Consider, on the sphere $Y$, the meromorphic 1-form

$$
\omega=\prod_{i=1}^{n}\left(t-t_{i}\right)^{\left(a_{i}+b_{i}\right) / 2} d t .
$$


Lift it via the hyperelliptic covering projection to $Z$ and call the lift $d h$. Clearly, this meromorphic 1-form has the required zeroes (and poles) at the preimages of the $t_{i}$.

The periods of $d h$ are all computable as periods of the form $\omega$ on $Y$ which are all computable as $2 \pi i$ multiples of purely real residues.

This lemma will apply to all cases needed. We deduce:

Theorem 3.3.5. Let $X_{1}$ and $X_{2}$ be reflexive orthodisks with exponents $a_{i}$ and $b_{i}$. Suppose that $a_{i}+b_{i} \equiv 0(\bmod 2)$. Then the above Weierstrass data define a minimal surface.

Proof. It remains to show that the Weierstrass data thus found have purely imaginary periods, or equivalently (see formula $(2.2)$ ) that the forms $G d h$ and $G^{-1} d h$ have conjugate periods. Yet the forms $G d h$ and $G^{-1} d h$ on the Riemann surface $Z$ are lifted from the canonical forms on the orthodisks $X_{1}$ and $X_{2}$, respectively; the conjugacy of the orthodisks $X_{1}$ and $X_{2}$ then forces the periods of $G d h$ and $G^{-1} d h$ on the Riemann surface $Z$ to be conjugate.

Definition 3.3.6. We call the pair of vertex data $a_{i}, b_{i}$ of a pair of orthodisks of the same genus formal Weierstrass data.

3.4. Geometric significance of the formal Weierstrass data. In this section we assume that we have a pair of reflexive orthodisks (i.e., conjugate orthodisks defined over the same underlying conformal ploygon) and we then determine the geometric data of the resulting minimal surface. These data will be given in terms of the formal Weierstrass divisor data $a_{j}$ and $b_{j}$.

First, by construction, the Riemann surface resulting from a pair of conformal orthodisks with $n=2 g+1$ vertices will have genus $g$.

Recall (§2.2) that the Riemannian metric of the associated minimal surface with Weierstrass data $G$ and $d h$ is given by

$$
d s=\left(|G|+\frac{1}{|G|}\right)|d h| .
$$

Singularities of this metric can only occur at the $2 g+2$ vertices of the conformal polygon, where the branching (of the surface $Z$ over the double $Y$ ) occurs. By the divisor discussion, the one-form $d h$ will have a zero of order $1+\frac{a_{i}+b_{i}}{2}$ and $G$ a zero of order $\frac{a_{i}-b_{i}}{2}$. Hence $|G|+\frac{1}{|G|}$ will have a pole of order $\frac{\left|a_{i}-b_{i}\right|}{2}$ and thus to have a complete metric it is necessary and sufficient that

$$
2+a_{i}+b_{i} \leq\left|a_{i}-b_{i}\right|
$$

for all $i$ (including the vertex at infinity!). 
It is also easy to compute the total curvature or equivalently the degree of the Gauss map as

$$
\operatorname{deg} G=\frac{1}{2} \sum_{a_{i}>b_{i}} a_{i}-b_{i}
$$

where the index $i=\infty$ is possibly included.

In the following table we compile a list of typical special points on minimal surfaces and the resulting vertex data. Formal Weierstrass data are then given as ordered lists of points of these types, and these types only. In particular, these special points appear as vertices on the conformal polygons, with vertex data given as fractions of cone angles for the flat structures of the forms $\left(G d h, G^{-1} d h, d h\right)$. The fractions arise because a neighborhood of a special point on the Riemann surface may $n$-fold cover a neighborhood of its image on the conformal polygon, so that the cone angle on the conformal polygon will be $\frac{1}{n}$ of the cone angle on the Riemann surface. Formal Weierstrass data are conformal polygons labelled with vertex data chosen from this table.

Denote by $H$ finite nonbranch points of the minimal surface where the symmetry curves intersect, by $C$ a catenoid end, by $P$ a planar end with degree of the Gauss map equal to 3 , and with $R$ a point which lies on just one symmetry arc. An up-arrow $(\uparrow)$ means that the Gauss map takes the value $\infty$, a down-arrow $(\downarrow)$ that the Gauss map takes the value 0 . Then the divisors at the respective points are given by the table below:

$\begin{array}{ccccccccc} & G & d h & G d h & G^{-1} d h & L G d h & L G^{-1} d h & \operatorname{ex}(G d h) & \operatorname{ex}\left(G^{-1} d h\right) \\ H \uparrow & -1 & 1 & 0 & 2 & \pi / 2 & 3 \pi / 2 & -1 & 1 \\ H \downarrow & 1 & 1 & 2 & 0 & 3 \pi / 2 & \pi / 2 & 1 & -1 \\ C \uparrow & -1 & -1 & -2 & 0 & -\pi / 2 & \pi / 2 & -3 & -1 \\ C \downarrow & 1 & -1 & 0 & -2 & \pi / 2 & -\pi / 2 & -1 & -3 \\ P \uparrow & -3 & 1 & -2 & 4 & -\pi / 2 & 5 \pi / 2 & -3 & 3 \\ P \downarrow & 3 & 1 & 4 & -2 & 5 \pi / 2 & -\pi / 2 & 3 & -3 \\ R \uparrow & -1 & 1 & 0 & 2 & \pi & 3 \pi & 0 & 2 \\ R \downarrow & 1 & 1 & 2 & 0 & 3 \pi & \pi & 2 & 0\end{array}$

The last two columns just contain the vertex data $a_{i}$ and $b_{i}$ of the formal Weierstrass data for $G d h$ and $G^{-1} d h$. The last two rows record angles that are not quite in the same pattern as the previous rows; the points $R$ have a surrounding neighborhood with only a two-fold symmetry while the points $H$, $C$, and $P$ all have neighborhoods with a four-fold symmetry. These points will only be important in $\S 8.2$ when we indicate how one might deform the surfaces with planar ends into those with catenoid ends. (Full details will be given in [WW2].) 
3.5. Examples of simple orthodisks. In this section we give some motivating examples of minimal surfaces and their corresponding orthodisks. We begin first with some discussion about moduli spaces of orthodisks, which we shall later formalize at the outset of $\S 4$. We then organize our examples into families, depending upon whether the associated moduli spaces are empty, singletons, or nontrivial.

To begin then, observe that from some formal Weierstrass data, we may draw orthodisk systems consisting of conjugate orthodisks $\Omega_{G d h}$ and $\Omega_{G^{-1}} d h$ for the forms $G d h$ and $G^{-1} d h$, respectively. We often have some freedom in deciding the lengths of the edges of the orthodisks, even up to equivalence of conformal polygons. Thus, the formal Weierstrass data determine a moduli space of conjugate orthodisk systems $\left\{\Omega_{G d h}, \Omega_{G^{-1} d h}\right\}$. While the function theory of this moduli space is the principal subject of this paper, here we shall content ourselves with a few examples for which the moduli space is either trivial or small. (See [WW2] for more examples.)

\subsubsection{A singleton moduli space.}

Example 3.5.1. The Costa surface $\mathrm{DH}_{0,0}\left(=C T_{0}\right)$. This surface is the starting point for the investigations in this paper. We seek a torus with two catenoid ends $\left(C_{1}\right.$ and $\left.C_{2}\right)$, one planar end $P_{1}$ and one finite point $H_{1}$ with vertical normal. We also assume our standard eight symmetries: reflections about two orthogonal vertical planes and a rotation about a horizontal line. (Previous existence and uniqueness proofs may be found in [Cos] and [Ho-Me].) From the table in $\S 3.4$, we can write formal Weierstrass data for this surface, assuming that the points occur on the real line in the order $C_{1}-P_{1}-C_{2}$ $-H_{1}$. We will justify this assumption in $\S 3.6$; however, below we reproduce a computer image of a fundamental domain of Costa's surface for the group generated by reflections in vertical planes. This makes apparent the conformal polygon, and the order of the special points on its boundary.

A table corresponding to the one in $\S 3.4$ is given below; it computes the cone angles for the forms $G d h$ and $G^{-1} d h$ on the putative conformal polygon for Costa's surface. These flat structures are drawn below, assuming an additional symmetry about the line $\{y=-x\}$.

$\begin{array}{ccccccccc} & G & d h & G d h & G^{-1} d h & L G d h & L G^{-1} d h & \operatorname{ex}(G d h) & \operatorname{ex}\left(G^{-1} d h\right) \\ C_{1} \downarrow & 1 & -1 & 0 & -2 & \pi / 2 & -\pi / 2 & -1 & -3 \\ P_{1} \uparrow & -3 & 1 & -2 & 4 & -\pi / 2 & 5 \pi / 2 & -3 & 3 \\ C_{2} \downarrow & 1 & -1 & 0 & -2 & \pi / 2 & -\pi / 2 & -1 & -3 \\ H_{1} \downarrow & 1 & 1 & 2 & 0 & 3 \pi / 2 & \pi / 2 & 1 & -1\end{array}$

Yet recall that any conformal quadrilateral with a symmetry across a diagonal is conformally equivalent to a square. 


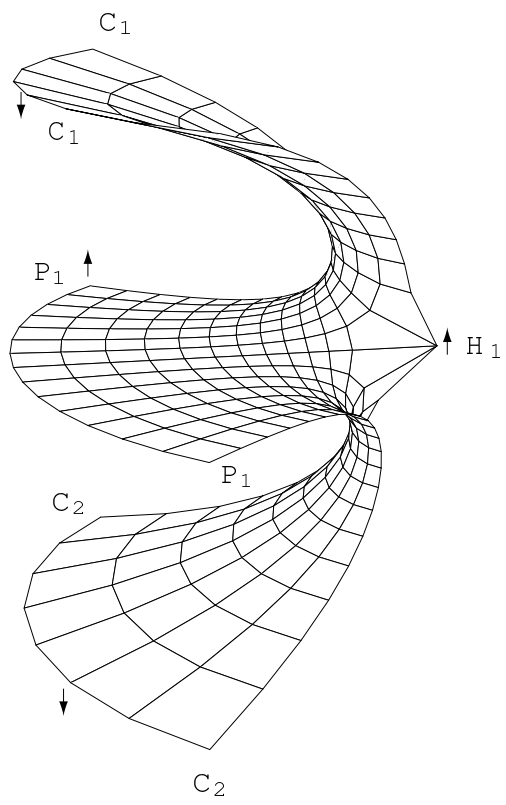

A quarter of Costa's surface - a conformal image of the orthodisks

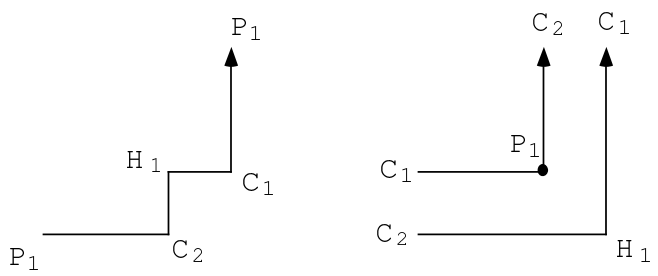

Costa orthodisks

Thus we then note that (as in Example 3.5.1), the moduli space of possible examples consists only of the singleton of a pair of square tori, so that the only element in the moduli space is a reflexive pair. This establishes the existence of this surface, by a proof that is somewhat distinct from the other proofs of existence of this surface ([Cos], [Ho-Ka] $)$.

\subsubsection{Empty moduli spaces.}

Nonexample 3.5.2(i). Catenoid with one handle. In this example, we try to construct a minimal surface with two catenoid ends and one handle. Now, a theorem of Schoen [Sch] implies that any minimal surface of genus $g \geq 1$ with but two embedded catenoid ends cannot exist; here we will require in addition 
the eightfold symmetry present in all of our examples. Yet, it is clear from an analysis of the orthodisks pictured below that such a surface cannot exist with 4 -fold symmetry, because there is no symmetric and conjugate pair: the periods from $\overline{C_{1} C_{2}}$ to $\overline{H_{1} H_{2}}$ are conjugate about the line $\{y=-x\}$, while the periods from $\overline{C_{2} H_{2}}$ to $\overline{H_{1} C_{1}}$ are conjugate across the line $\{y=-x\}$.

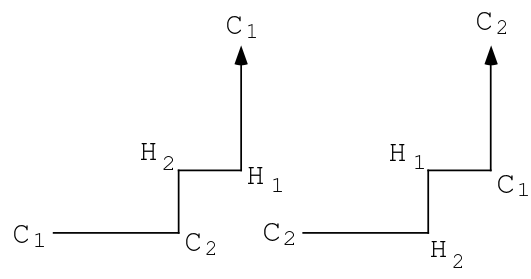

Catenoid with one handle

We will use this technique of showing nonexistence extensively when we prove the nonexistence parts of the main theorem.

Nonexample 3.5.2(ii). The Horgan surface $\mathrm{DH}_{0,1}$. The second example is called the Horgan surface (see [Ho-Ka]): To visualize it, start with one plane and two handles growing upward and downward and perpendicular to each other. Both handles connect to catenoid ends. This looks almost as shown in the following figure:

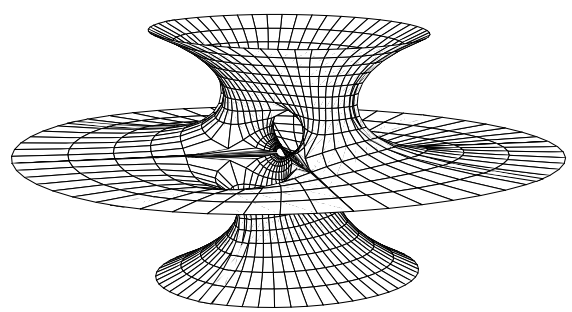

The Horgan surface?

This pattern leads to a sequence

$$
C_{1}-P-C_{2}-H_{2}-M-H_{1}-C_{1}
$$

(see $§ 3.6)$ where $M$ denotes a previously unencountered regular point where the symmetry lines cross. The orthodisks are as follows: 

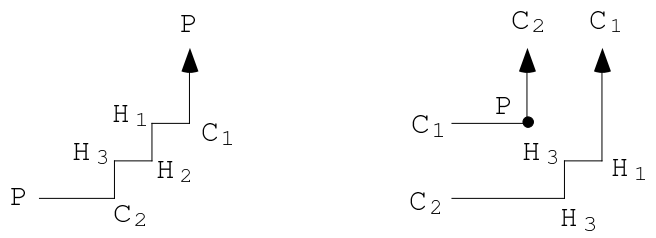

Horgan orthodisk

Recently, one of us [W] showed that this surface cannot exist by a method that is different from that used in Example 3.5.2(ii). Therefore, this moduli space is empty. We find it remarkable that our general existence proof fails at precisely one point for the case of this surface; see $\S 4$, after Definition 4.1.1.

3.6. Orthodisks for the Costa towers. This section is intended to give a heuristical picture for the Costa towers and to derive the orthodisk pairs from this picture.

As a first series of new examples, we introduce the orthodisks for generalized Costa surfaces which we call Costa towers:

Definition 3.6.1. A Costa tower of genus $g$ is a complete minimal surface $C T_{g}$ of genus $g$ with two parallel catenoid ends and $g$ planar ends which are all embedded. These surfaces have total curvature $4 \pi(g+2)$.

Remark 3.6.2. The existence of generalized Costa surfaces is known for $g \leq 2$ and numerical evidence has been provided for $g=3$; see [Cos], [Bo], [HoMe], [Woh1], [Woh2]. All these examples have (at least) a two-fold reflectional symmetry at two perpendicular planes intersecting in the $z$-axis. In their construction, this assumption makes the period problem low dimensional. The genus 1 Costa surface has the square torus as underlying Riemann surface and is known to admit a deformation to all rectangular tori such that the planar end becomes catenoidal.

We will briefly discuss these deformations from our point of view in $\S 8.2$.

To get an impression of how these surfaces might appear, imagine cutting a catenoid by $g$ horizontal planes. For each plane, we have to resolve a singularity which looks topologically like a horizontal plane cut by a vertical cylinder. We replace a neighborhood of the singular locus in the complex of cylinder and plane by one of the two shapes below; i.e., we replace the upper part of the cylinder by a $Y$-piece whose lower two boundary components are glued to two holes in the plane and glue the boundary component of the lower part of the cylinder to another hole in the plane in between the two first holes, and finally glue the boundary of the flat planar piece to the planar boundary of the excised neighborhood. 

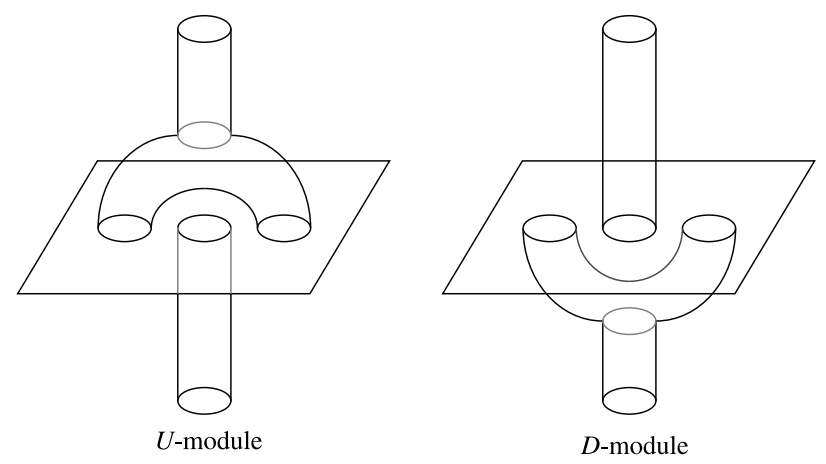

Building blocks for informal surface models

We will call the left gadget a $U$-module (for $u p$ ) and the right one a $D$ module (for down). It is convenient to regard the planar portions of the figures as extending indefinitely.

Stacking $g$ copies of these modules over each other and finishing with catenoidal ends yields a rough informal model of candidates for a Costa tower of genus $g$. These models are not very realistic, but they will suffice to write down candidate formal Weierstrass data for the surfaces.

Remark 3.6.3. At first glance there are two more possible constructions of this type, which are obtained by rotating the above pieces by $90^{\circ}$ around the $z$-axis, but it turns out that these give no new Weierstrass data, so we will neglect them from the beginning.

Despite this, there are still many possible ways to attach these modules to each other, but we will not attempt to discuss all of them. Only for a very distinctive construction will our proof establish the existence of minimal surfaces corresponding to these models, and we have presently no contribution to make regarding the others. For these other models, no complete existence or nonexistence proofs are known, but numerical experiments make it doubtful that other module towers will produce more minimal surfaces.

Now we proceed to derive the Weierstrass candidate data defined by a module tower. Cutting a generalized Costa surface composed of these modules by its two vertical symmetry planes decomposes it into four congruent simply connected domains which we want to describe using orthodisks. The first step is to recover the formal Weierstrass data from the putative geometry:

Lemma 3.6.4. Let $X$ be a generalized Costa surface consisting of $g$ modules $X_{i}$ of type $U$. Denote by $P_{i}$ the point on $X$ corresponding to the planar end of module $i$ and by $H_{i}$ the saddle point of that module $X_{i}$. Denote by $G$ the Gauss map and by dh the height differential of $X$. Then the divisors of $G$ and $d h$ are given by the table below: 


$$
\begin{aligned}
(G) & =C_{1}^{1} P_{1}^{-1} H_{1}^{1} P_{2}^{1} H_{2}^{-1} \cdots P_{g}^{(-1)^{g}} H_{g}^{-(-1)^{g}} C_{2}^{-(-1)^{g}} \\
(d h) & =C_{1}^{-1} P_{1}^{1} H_{1}^{1} P_{2}^{1} H_{2}^{1} \cdots P_{g}^{1} H_{g}^{1} C_{2}^{-1} \\
(G d h) & =H_{1}^{2} P_{2}^{2} \cdots \\
\left(G^{-1} d h\right) & =C_{1}^{-2} P_{1}^{2} H_{2}^{2} \cdots .
\end{aligned}
$$

Proof. First we determine the direction of the normal vector at all of the vertical points of $G$. We can assume that the outer normal vector at the upper catenoid end $C_{1}$ is pointing downward. As can be read off from the above figure, the normal will switch between up and down from planar end to planar end. Hence all odd labeled planar ends will have an upward pointing normal, all even planar ends a downward pointing normal, and the bottom catenoid end has normal pointing up if and only if $g$ is even. Again by the figure above, the normal at the saddle point of a module point is always in the opposite direction as the normal at the planar end. Using the information from the table at the end of $\S 3.4$, we get the claimed divisors.

Definition 3.6.5. Let $T$ be a sequence of length $g$ consisting of symbols $U$ and $D$. A surface of type $T$ is a generalized Costa surface consisting of modules $X_{i}$ where the handle of module $i$ grows upward (resp. downward) if the symbol $i$ is $U$ (resp. $D$ ).

Lemma 3.6.6. Let $X$ be a surface of type $T$. Then the formal Weierstrass data are explicitly determined by the type $T$.

Proof. To see this, we first consider a surface where all modules are $U$ modules. We follow the symmetry line given by the intersection with the $y=0$-plane, beginning at the top catenoid end on the left. This line goes down to the first planar end to the left. There the total angle is divided into four equal pieces by the two symmetry lines meeting there. We continue now on the symmetry line defined by the $x=0$-plane which goes down into the second planar end. Continuing this process and switching to another symmetry line at each vertex, we descend through all planar ends until we reach the bottom catenoid end. From there, we ascend through all handles back to the top catenoid end. We denote this closed path by

$$
C_{1} \rightarrow P_{1} \rightarrow P_{2} \rightarrow \ldots \rightarrow P_{g} \rightarrow C_{2} \rightarrow H_{g} \rightarrow \ldots \rightarrow H_{2} \rightarrow H_{1} \rightarrow C_{1} .
$$

Now exchange the $k^{\text {th }}$ module by a $D$-module. As can be seen from the figure above, this affects only the entries $P_{k}$ and $H_{k}$ in the path list; they are just exchanged. Hence for an arbitrary surface $X$ of type $D$ we get a path sequence 


$$
C_{1} \rightarrow A_{1} \rightarrow A_{2} \rightarrow \ldots \rightarrow A_{g} \rightarrow C_{2} \rightarrow B_{g} \rightarrow \ldots \rightarrow B_{2} \rightarrow B_{1} \rightarrow C_{1}
$$

where $A_{k}=P_{k}$ and $B_{k}=H_{k}$ if the module $X_{k}$ is of type $U$ and $A_{k}=H_{k}$ and $B_{k}=P_{k}$ otherwise.

We now give some low genus examples to illustrate the domains. The images are developed images of the flat structures $\Omega_{G d h}$ and $\Omega_{G^{-1} d h}$ for $G d h$ and $G^{-1} d h$ (respectively) on the Riemann surfaces: the domain is locally always to the left of the curve. We indicate the corners with angle $5 \pi / 2$ by a fat dot.

The genus 1 example using just one handle is the Costa surface; see Example 3.5.1.

For genus 3, there are essentially two distinct possible symmetric module sequences with types $(U U U)$ and $(U D U)$; i.e., there are only these two up to replacing a ' $U$ ' by a ' $D$ ' and vice versa.
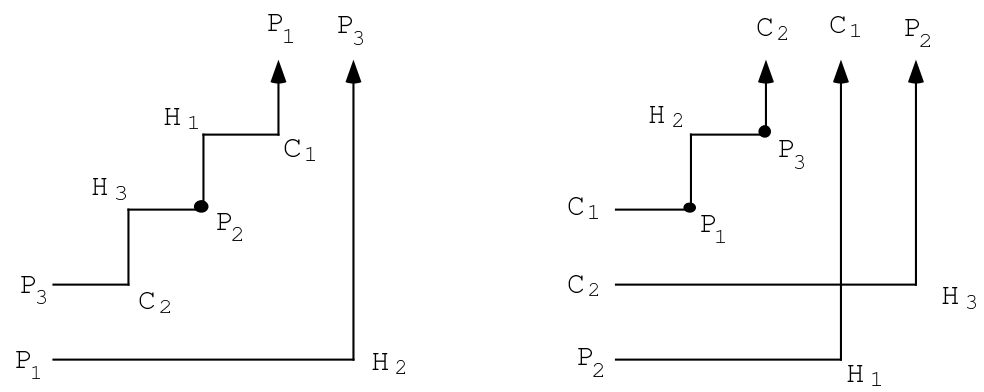

Orthodisks for the $U U U$ candidate surface
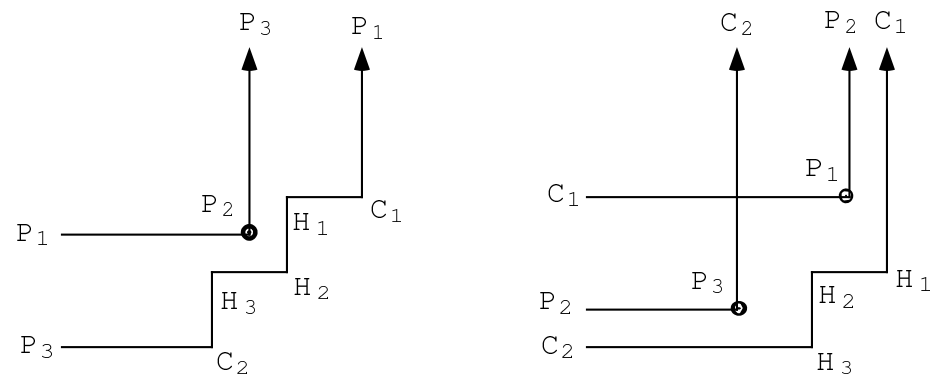

Orthodisks for the $U D U$ candidate surface

We will only be able to prove the existence of the second one. Numerical investigations give little hope that the first one also exists. Starting with the second surface, we will inductively construct all our other examples. 
To see how the surface of type $T=(U D)^{n} U$ evolves as $n$ increases, here are the orthodisks for $U D U D U$ :
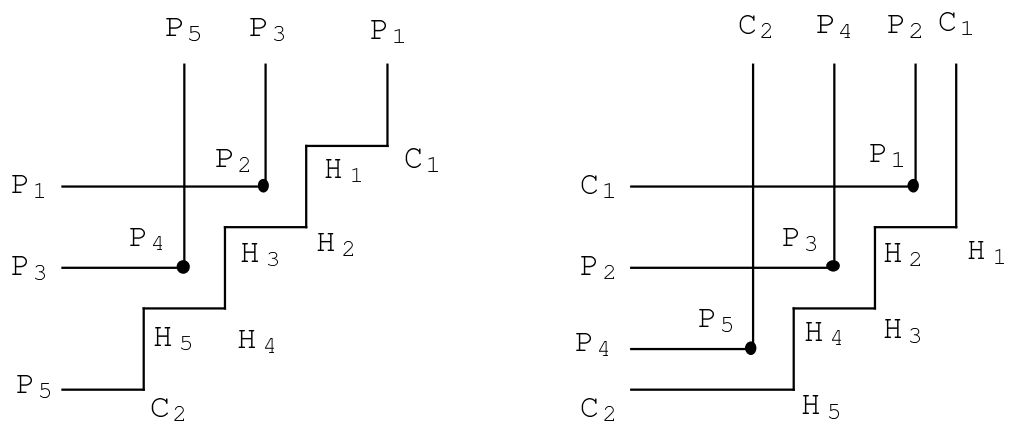

The $U D U D U$ candidate orthodisks for a genus 5 surface

Geometrically, our choice of candidate surfaces can be characterized by the property that the intersection of the surface with the symmetry planes contains one arc component which connects all the finite points with vertical normal (on the $z$-axes) while all other arc components connect the ends.

The pair of orthodisks for the general surface of type $C T_{n}=(U D)^{n} U$ surface of genus $g=2 n+1$ are indicated below. These surfaces have 2 catenoid ends denoted by $C_{1}, C_{2} ; g$ planar ends denoted by $\left\{P_{k}\right\}$; and $g$ finite points with vertical normals at the handles denoted by $\left\{H_{k}\right\}$. There are $4 n+4$ vertices in each orthodisk, of which $n$ are branch points.

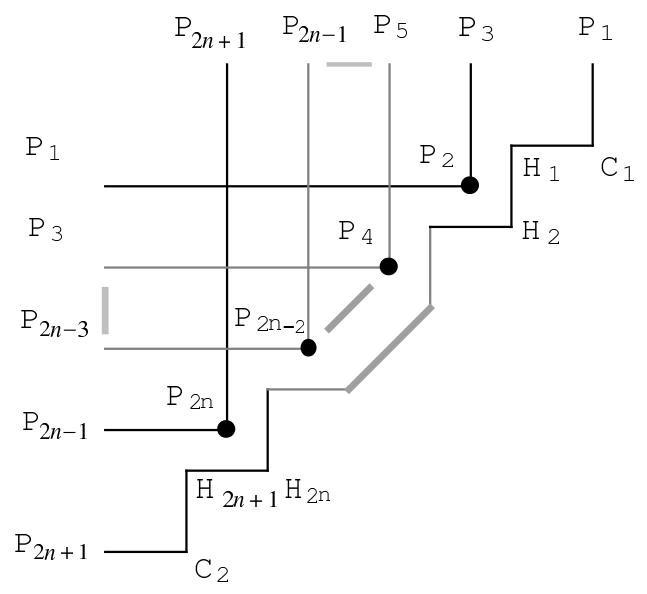

The $\Omega_{G d h}$ orthodisk for $C T_{n}$ 


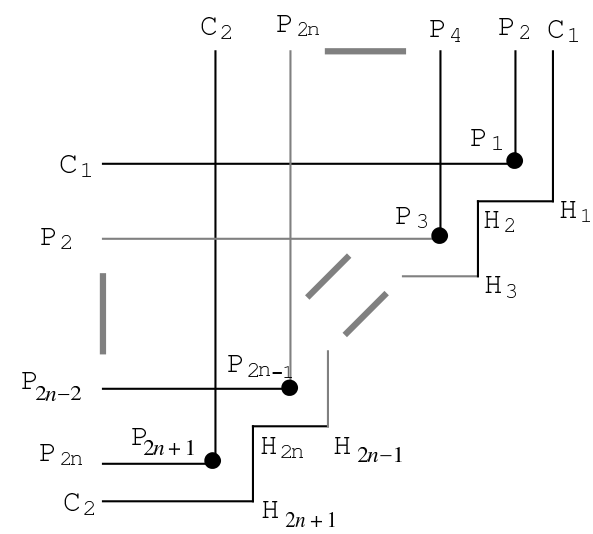

The $\Omega_{G^{-1} d h}$ orthodisk for $C T_{n}$

3.7. More orthodisks by drilling holes. In the previous section, we have described the candidate formal Weierstrass data of a family of minimal surfaces generalizing Costa's surface for every odd genus greater than 1 . Now we are going to add handles (without enlarging the symmetry group) to all these surfaces in a symmetric fashion. We call this process hole drilling, and we will designate the resulting surfaces as $\mathrm{DH}_{m, n}$ surfaces.

We start with an informal description. For each odd integer $n \geq 3$ we describe a process of handle addition which inductively adds to the surface of type $(U D)^{n} U$ an arbitrary number of handles without adding ends. Recall that our Costa-tower surfaces are composed of a stack of modules called $U$ and $D$-module.

Now we allow two other kind of modules, called $F$ - and $S$-module (for front and side) which are vertical cylinders with handles drilled through them from the front or the side, as indicated:

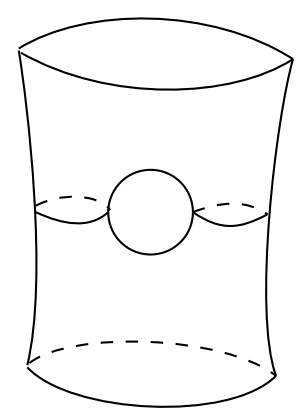

$F$-module

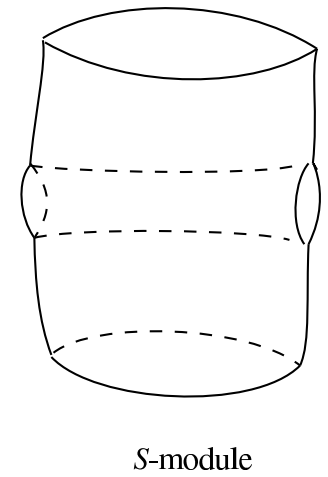

$S$-module

Modules for drilling holes 
As in the case of the $U$ - and $D$ - modules, we are not able to prove existence for surfaces made of arbitrary module sequences.

As for the Costa towers, our choice of candidate surfaces is characterized by the property that the intersection of the surface with the symmetry planes contains one arc component which connects all the finite points with vertical normal (on the $z$-axes) while all other arc components connect the ends.

To be more specific, we will now add one handle to the $U D U$-surface.

There are apparently two possibilities according to the above rule, namely $U S D U$ and $U D F U$. But it is easy to see that the module sequences $S D$ and $D L$ generate equivalent formal Weierstraß data: If one follows the intersection curves of these module sequences with the vertical symmetry planes, one obtains the same pattern of special points at the $z$-axes, hence the same formal Weierstrass data. So the topological significance of these modules does not impose much restriction on the possible geometry of the surfaces.

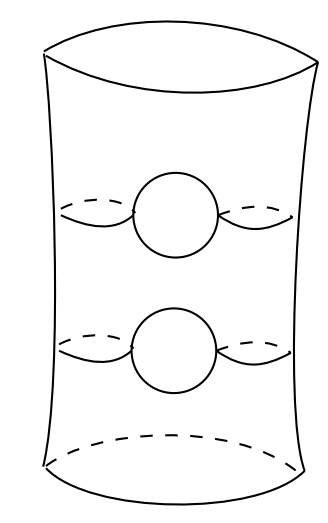

Iterated hole drilling

Remark 3.7.1. This process does work formally for $n=1$, but the candidate surface would be Costa's surface with one handle added; this is known as the Horgan surface. See Nonexample 3.5.2(ii).

We now describe the orthodisks arising from the handle addition process. A module sequence of type $U F$ or $D U$ results in two consecutive finite corners of angle $\pi / 2$ and $3 \pi / 2$ (or vice versa) of the orthodisks. Inserting a handle according to the above rules results in adding two new finite corners in between the old corners in a zigzag fashion. We illustrate this for $U S D U$ (compare with Example 3.5.1). 

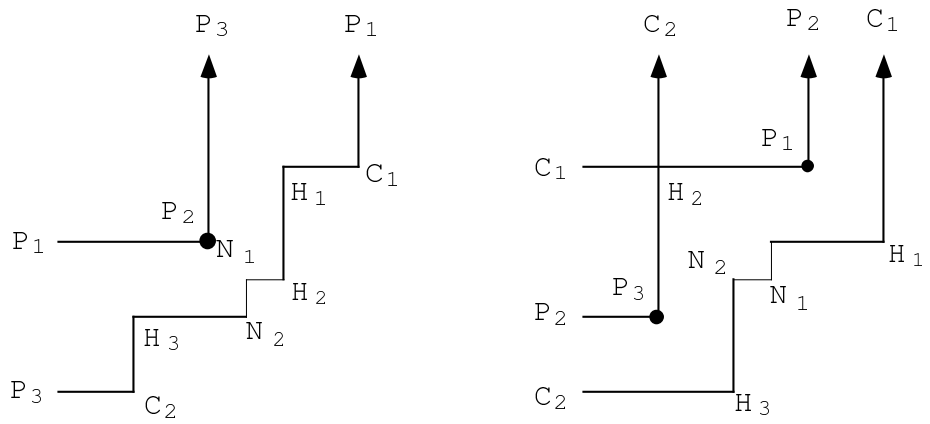

Growing a handle by adding the two new corners $N_{1}$ and $N_{2}$

Now that we have introduced these new module sequences, we can extend our original one-parameter sequence of Costa towers to a two-parameter sequence of orthodisks. We introduce a new two-parameter family of orthodisks and give geometric coordinates. To set notation, we consider a pair of orthodisks with $2 n+1$ vertical normals at the $z$-axis and $2 m+1$ planar ends. Together with the two catenoid ends we have $2 m+2 n+4$ Weierstraß points so that the Riemann surface will have genus $m+n+1$. In the case $m=n$ we are in the situation of the Costa tower surfaces of type $(U D)^{n} U$. We are aiming now for $n>m \geq 0$, the cases in which we will establish existence in the next three sections.

Here is a table depicting these surfaces and giving their historical context:

$\begin{array}{ccccccccc}m \backslash n & 0 & 1 & 2 & 3 & 4 & 5 & 6 & 7 \\ 0 & \text { Costa } & \text { Horgan } & ? & ? & ? & ? & ? & ? \\ 1 & - & B-W & + & + & + & + & + & + \\ 2 & - & - & + & + & + & + & + & + \\ 3 & - & - & - & + & + & + & + & + \\ 4 & - & - & - & - & + & + & + & + \\ 5 & - & - & - & - & - & + & + & +\end{array}$

Meaning of the symbols:

(1) ?: We have not been able to construct these surfaces. Constructing one would imply the existence of the others with higher genus. We conjecture that these do not exist. ${ }^{2}$

(2) -: We will see that these do not exist. This is a very special case of the Hoffman-Meeks conjecture, and is the second portion of the Main Theorem B.

${ }^{2}$ Added in Proof: This conjecture was confirmed in [MW]. 
(3) + : Dealt with in the following sections, this is the first portion of the main theorem.

(4) $B-W$ : This surface was found numerically by Boix and Wohlgemuth; see $[\mathrm{Bo}]$.

\section{The space of orthodisks}

4.1. Introduction. In this chapter, we will parametrize the space of pairs of conjugate orthodisks by geometric data, set up a height function on this space which measures the conformal distance between the two orthodisks and prove its properness. The main point in choosing the geometric coordinates lies in what we call the completeness condition: We require that whenever we have a sequence of points leaving every compact set of the coordinate space (that is, when a geometric degeneration occurs), at least one of the two orthodisks $\Omega_{G d h}$ and $\Omega_{G^{-1} d h}$ degenerates conformally. After introducing complete geometric coordinates, the height function is set up to measure differences in the extremal lengths of cycles in a way which detects precisely the degenerations coming from a geometric degeneration. Because it can easily happen that a geometric degeneration forces the simultaneous conformal degeneration of both orthodisks, we also need to measure the rates of growth or decay of extremal lengths. This is done by a technical monodromy argument which is postponed to the final section 4.7. For the sake of clarity, we will now describe the height function and formulate the monodromy theorem.

The height is constructed as a sum of terms, each of which measures the possible conformal degeneration of the extremal length of one cycle:

Definition 4.1.1. Fix formal Weierstrass data and consider a cycle $c$ in a conformal disk. Given a pair of conjugate orthodisks $\Omega_{G d h}$ and $\Omega_{G^{-1}}$ dh with the chosen formal Weierstrass data, define the height of a cycle $c$ by

$$
\mathcal{H}(c)=\left|e^{1 / \operatorname{Ext}_{\Omega_{G d h}}(c)}-e^{1 / \operatorname{Ext}_{\Omega_{G}-1}(c)}\right|^{2}+\left|e^{\operatorname{Ext}_{\Omega_{G d h}}(c)}-e^{\operatorname{Ext}_{\Omega_{G}-1}(c)}\right|^{2} .
$$

The definition of the height requires a choice of cycles $c_{i}$ and will be defined as

$$
\mathcal{H}=\sum_{i} \mathcal{H}\left(c_{i}\right)
$$

This choice is restricted by two different requirements:

(1) Asymptotic computability. To prove properness, we need a precise asymptotic formula in the case of geometric degenerations. Such formulas are available only in special situations. For us, this means that we have to choose simple cycles or double cycles which are symmetric with respect to the diagonal. Such cycles will be called admissible cycles. 
(2) Avoidance of certain edges: In the next chapter, we need to compute the derivative of the height with respect to changes in the coordinates which can be described as 'pushing edges'. There, more cycles ending at the pushed edge yield more terms of the height function that we will need to control, so we aim not to have too many cycles meeting a particular given edge.

These restrictions have the curious effect that it becomes easier to choose the right cycles for surfaces of higher genus and with more topology; it is the lowest genus case which causes most problems. And in fact, the reason that our present proof does not extend to prove the existence of the nonexisting Horgan surface (see $[\mathrm{W}]$ ) is our inability to choose sufficiently many good cycles.

From the above form of the height function it is clear that we can prove properness when we can ensure that the extremal length of a chosen cycle tends to 0 or $\infty$ for one domain but remains bounded and bounded away from 0 for the other one. However, we will encounter situations where the extremal length of a cycle degenerates to zero (or infinity) simultaneously in both domains. Here we will need to invoke the following theorem, proven in $\S 4.7$ as Lemmas 4.7 .1 and 4.7 .2 by a monodromy argument:

Monodromy Theorem 4.1.2. Fix formal Weierstrass data and an admissible cycle $c$ (see above). Consider a sequence of pairs of conjugate orthodisks $\Omega_{G d h}$ and $\Omega_{G^{-1} d h}$ such that either $c$ encircles a single edge in $\Omega_{G d h}$ shrinking geometrically to zero and both $\operatorname{Ext}_{X_{1}\left(p_{n}\right)}(\gamma) \rightarrow 0$ and $\operatorname{Ext}_{X_{2}\left(p_{n}\right)}(\gamma)$ $\rightarrow 0$ or $c$ foots on an edge in $\Omega_{G d h}$ shrinking geometrically to zero and both $\operatorname{Ext}_{X_{1}\left(p_{n}\right)}(\gamma) \rightarrow \infty$ and $\operatorname{Ext}_{X_{2}\left(p_{n}\right)}(\gamma) \rightarrow \infty$. Then $\mathcal{H}(c) \rightarrow \infty$ as $n \rightarrow \infty$.

For the sake of a clear exposition, we have treated in detail the special but most fundamental case $C T_{3}=\mathrm{DH}_{1,1}$ in $\S 4.2$ and $\S 4.3$, postponing the general case to the two subsequent sections, $\S 4.4$ and $\S 4.5$. Historically, this surface was first discovered numerically by Boix and Wohlgemuth ([Bo]).

4.2. Geometric coordinates for the $\mathrm{DH}_{1,1}$ surface. In this section, we describe geometric coordinates for our basic surface $\mathrm{DH}_{1,1}$.

Denote

$$
\Delta=\left\{(y, b, g) \in \mathbb{R}^{3}: 0<y, b, g<1, y+b+g=1\right\}
$$

and by $\bar{\Delta}$ its closure. For each point in $\Delta$, we will associate a pair of orthodisks. We introduce three cycles, called yellow, green, and blue: 

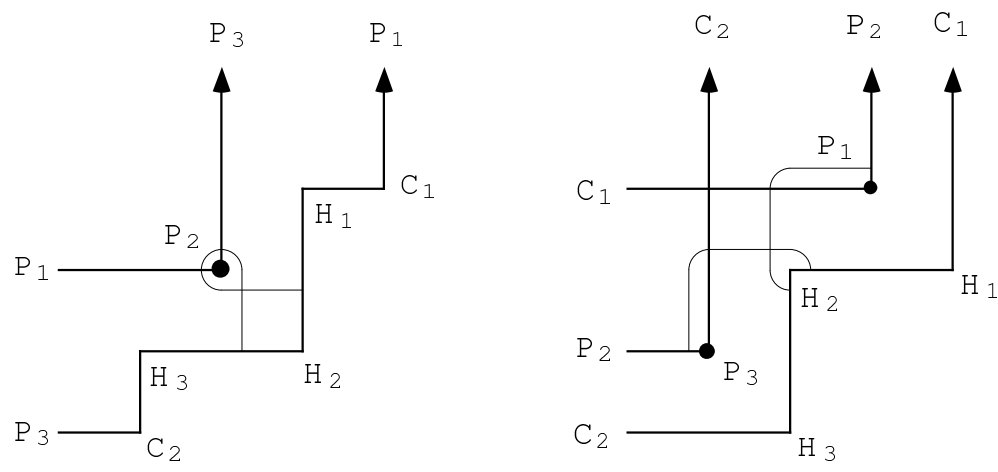

yellow cycle
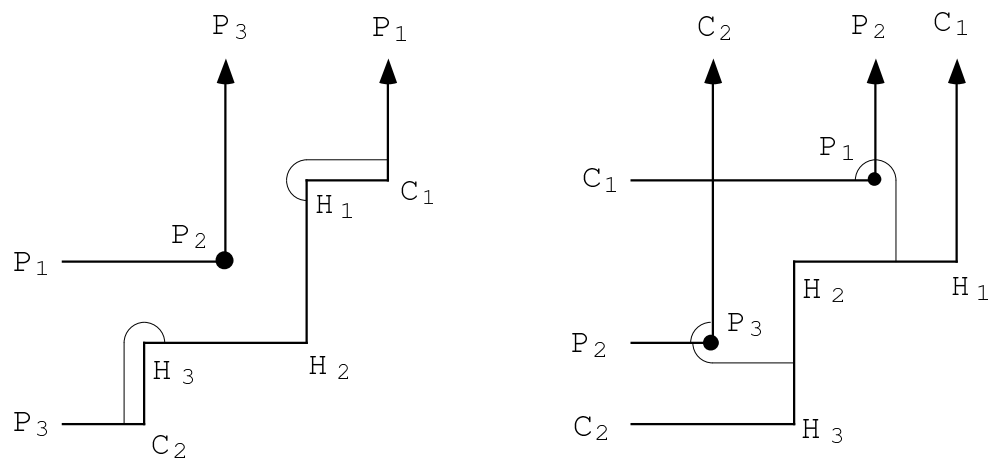

blue cycle
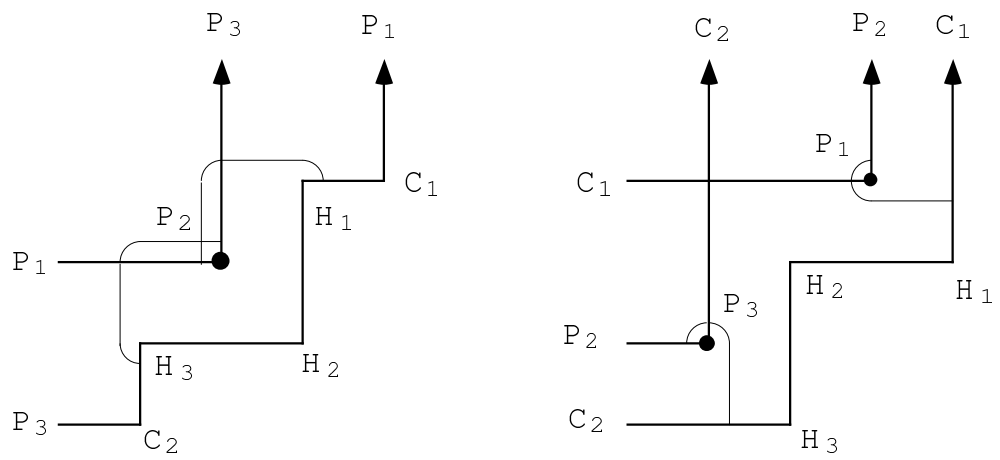

green cycle 
Formally,

(1) yellow consists of (undirected) arcs connecting $H_{3} H_{2}$ with $P_{1} P_{2}$ and symmetrically $\mathrm{H}_{2} \mathrm{H}_{1}$ with $\mathrm{P}_{2} \mathrm{P}_{3}$.

(2) blue consists of (undirected) arcs connecting $P_{3} C_{2}$ with $\mathrm{H}_{3} \mathrm{H}_{2}$ and symmetrically $C_{1} P_{1}$ with $H_{2} H_{1}$.

(3) green consists of (undirected) arcs connecting $P_{1} P_{2}$ with $H_{1} C_{1}$ and symmetrically $\mathrm{P}_{2} \mathrm{P}_{3}$ with $\mathrm{C}_{2} \mathrm{H}_{3}$.

Why should we choose these particular curves? At this stage, the choice has little to do with the eventual geometry of the minimal surface, and much to do with the conformal and combinatorial geometry of the orthodisk: our goal is to meet criteria (1) and (2) of the last subsection.

Now each orthodisk is determined up to scaling by the lengths of the periods of these cycles which we denote by $y, b, g$ subject to the condition $y+b+g=1$. Hence for any triple $(y, b, g) \in \Delta$ we can form a pair of orthodisks with these normalized period lengths. It is clear that these orthodisks will be conjugate.

We call $\Delta$ a geometric coordinate system for the formal Weierstrass data of type $\mathrm{DH}_{1,1}$. By this we mean the following:

Definition 4.2.1. Let formal symmetric Weierstrass data be given. A geometric coordinate system is an open subset $\Delta$ of a Euclidean space such that for each point in $\Delta$, there is a pair of normalized symmetric conjugate orthodisks with the given Weierstrass data such that the periods of the orthodisk are linear functions in the coordinates of the point in $\Delta$.

Here a pair of symmetric orthodisks is normalized if the outer sheet boundary of the orthodisk $\Omega_{G d h}$ consists of finite edges with total length equal to 1 .

We will from now on only consider normalized orthodisks.

Here our geometric coordinate system $\Delta$ records the periods for a set of cycles that span the homology of the (covering) surface, hence provides sufficient information for determining all of the periods via linear functions of the given ones.

In the rest of this section we will show that our coordinate geometric system is complete in the following sense:

Definition 4.2.2. A geometric coordinate system $\Delta$ is called complete if for any sequence of points in $\Delta$ leaving any compact set, the conformal structure of at least one of the orthodisks degenerates.

This condition will ensure that our height function satisfies the minimal necessary requirements for being proper. 
Lemma 4.2.3. The above geometric coordinate system $\Delta$ for $\mathrm{DH}_{1,1}$ is complete.

Proof. Consider a sequence $p_{n} \in \Delta$ with $p_{n} \rightarrow \partial \Delta$. After choosing a subsequence, we can assume that $p_{n} \rightarrow p_{0} \in \partial \Delta$ Now we want to detect conformal degenerations of the orthodisk domains. For this it will be convenient to use the extremal lengths of the cycle blue and another cycle mauve introduced below:
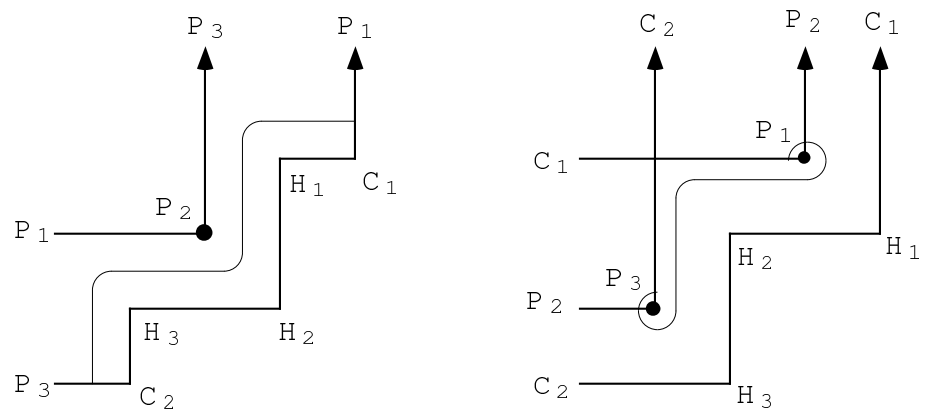

\section{mauve cycle}

Mauve consists of curve connecting $P_{3} C_{2}$ with $C_{1} P_{1}$.

Denote the extremal length of a cycle (say blue) in (say) the orthodisk $\Omega_{G d h}$ as usual by $\operatorname{Ext}_{\Omega_{G d h}}$ (blue).

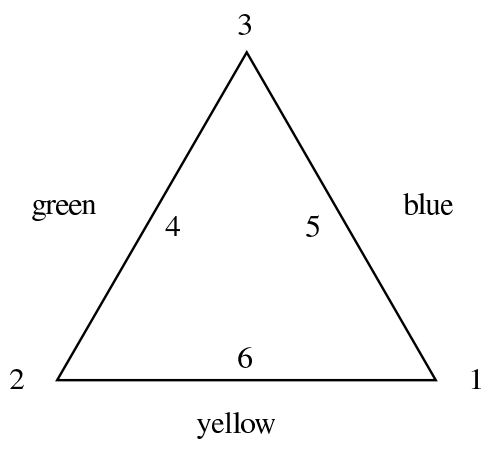

Geometric coordinate simplex 
We need to distinguish six cases (which are indicated in the above figure), depending on the location of $p_{0}$ in $\partial \Delta$ :

(1) $p_{0}=(0,0,1): \operatorname{Ext}_{\Omega_{G d h}}($ blue $)=0$,

(2) $p_{0}=(0,1,0): \operatorname{Ext}_{\Omega_{G^{-1}} h}($ blue $)=\infty$,

(3) $p_{0}=(1,0,0): \operatorname{Ext}_{\Omega_{G d h}}($ blue $)=0$,

(4) $p_{0} \in(>0,>0,0): \operatorname{Ext}_{\Omega_{G^{-1}} h}($ mauve $)=\infty$,

(5) $p_{0} \in(>0,0,>0): \operatorname{Ext}_{\Omega_{G d h}}($ blue $)=0$,

(6) $p_{0} \in(0,>0,>0): \operatorname{Ext}_{\Omega_{G d h}}($ mauve $)=\infty$.

Since the degeneration of an extremal length clearly signals the degeneration of the conformal structure, we are done.

4.3. The height function for $\mathrm{DH}_{1,1}$. Our aim is to define a proper function on $\Delta$ which is zero if and only if the two domains $\Omega_{G d h}$ and $\Omega_{G^{-1} d h}$ given by the geometric coordinates of the point are conformal. The conformal difference between the two domains is naturally measured by expressions in the extremal length like the Teichmüller distance, which, however, is not proper.

To obtain properness, we need to measure rather subtle growth differences of extremal lengths.

Another important point for the choice of the height function is that we need to be able to decrease the height at noncritical points. This requires that we control the first derivative of the height, and this means heuristically that we should use as few curve families as possible in the definition of the height.

Definition 4.3.1. Consider on $\Delta$ the height function

$$
\begin{aligned}
\mathcal{H}_{1,1}= & \left|e^{1 / \operatorname{Ext}_{\Omega_{G d h}}(\text { blue })}-e^{1 / \operatorname{Ext}_{\Omega_{G}}{ }_{G_{d h}}(\text { blue })}\right|^{2} \\
& +\left|e^{1 / \operatorname{Ext}_{\Omega_{G d h}} \text { (mauve) }}-e^{1 / \operatorname{Ext}_{\Omega_{G}-1}(\text { mauve })}\right|^{2} \\
& +\left|e^{\operatorname{Ext}_{\Omega_{G d h}} \text { (blue) }}-e^{\operatorname{Ext}_{\Omega_{G}-1}(\text { blue })}\right|^{2} \\
& +\left|e^{\operatorname{Ext}_{\Omega_{G d h}} \text { (mauve) }}-e^{\operatorname{Ext}_{\Omega_{G}-1} \text { (mauve) }}\right|^{2} .
\end{aligned}
$$

Clearly the conformal structure of a domain $\Omega_{G d h}$ or $\Omega_{G^{-1} d h}$ is determined by the extremal lengths of blue and mauve, so that the height function is zero if and only if the two domains are conformal.

It is also clear that the height function admits at least the possibility of being proper, because at a boundary point, at least one of the extremal lengths used is either 0 or $\infty$ by Lemma 4.2.3. The main difficulty arises when 
the extremal lengths for the same cycle degenerate for both orthodisks. This requires that we measure extremal length growth rates and is responsible for the complicated shape of the height function.

We can now prove, up to the crucial monodromy, Theorem 4.1.2.

THEOREM 4.3.2. The above height function $\mathcal{H}_{1,1}$ is proper.

Proof. Consider a sequence $p_{n} \in \Delta$ with $p_{n} \rightarrow \partial \Delta$.

After choosing a subsequence, we can assume that $p_{n} \rightarrow p_{0} \in \partial \Delta$ and that all extremal lengths of blue and mauve converge to some numbers in $\mathbb{R} \cup\{0, \infty\}$.

By Lemma 4.2.3, at least one of the extremal lengths in the definition of the height function is 0 or $\infty$. Now observe that in the cases (3), (4) and

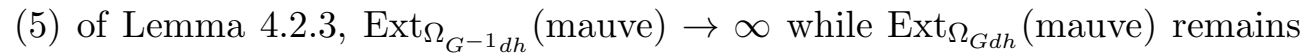

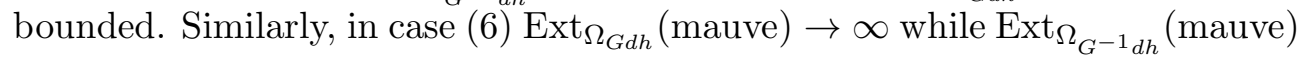
remains bounded. This leaves us with the cases (1) and (2). Here both mauve extremal lengths go to $\infty$.

The point now is that we are able to control the growth rates of the extremal length of blue. It then follows from Theorem 4.1.2 that, independently of the paths of approach to $\partial \Delta$, in case (1)

$$
\left|e^{1 / \operatorname{Ext}_{\Omega_{G d h}}(\text { blue })}-e^{1 / \operatorname{Ext}_{\Omega_{G}-1}(\text { blue })}\right|^{2} \rightarrow \infty
$$

while in case $(2)$

$$
\left|e^{\operatorname{Ext}_{\Omega_{G d h}}(\text { blue })}-e^{\operatorname{Ext}_{\Omega_{G^{-1}}} \text { (blue) }}\right|^{2} \rightarrow \infty .
$$

4.4. Geometric coordinates for the $\mathrm{DH}_{m, n}$ surfaces. The goal of this section is to introduce a set of coordinates for the moduli space of pairs of symmetric orthodisks with formal Weierstrass data of type $\mathrm{DH}_{m, n}$ and to prove that they are complete.

Fix formal Weierstrass data $a_{i}$ of a symmetric orthodisk of type $\mathrm{DH}_{m, n}$ for the rest of this section.

The corresponding pairs of orthodisks have the following geometry: The orthodisk $\Omega_{G d h}$ has an outer sheet bounded by a polygonal arc with finite vertices

$$
C_{2}-H_{2 n+1}-H_{2 n}-\ldots-H_{1}-C_{1}
$$

and $m$ interior sheets, each having one finite vertex, the branch point $P_{2 k}$. 
The orthodisk $\Omega_{G^{-1} d h}$ has an outer sheet bounded by a polygonal arc with finite vertices

$$
H_{2 n+1}-H_{2 n}-\ldots-H_{1}
$$

and $m+1$ interior sheets containing one finite branched vertex $P_{2 k+1}$ each.

A picture of such a pair of orthodisks is shown below for $\mathrm{DH}_{5,8}$. In the following figures, the orthodisk $\Omega_{G d h}$ will always be the left one. We discard the labeling of the vertices.
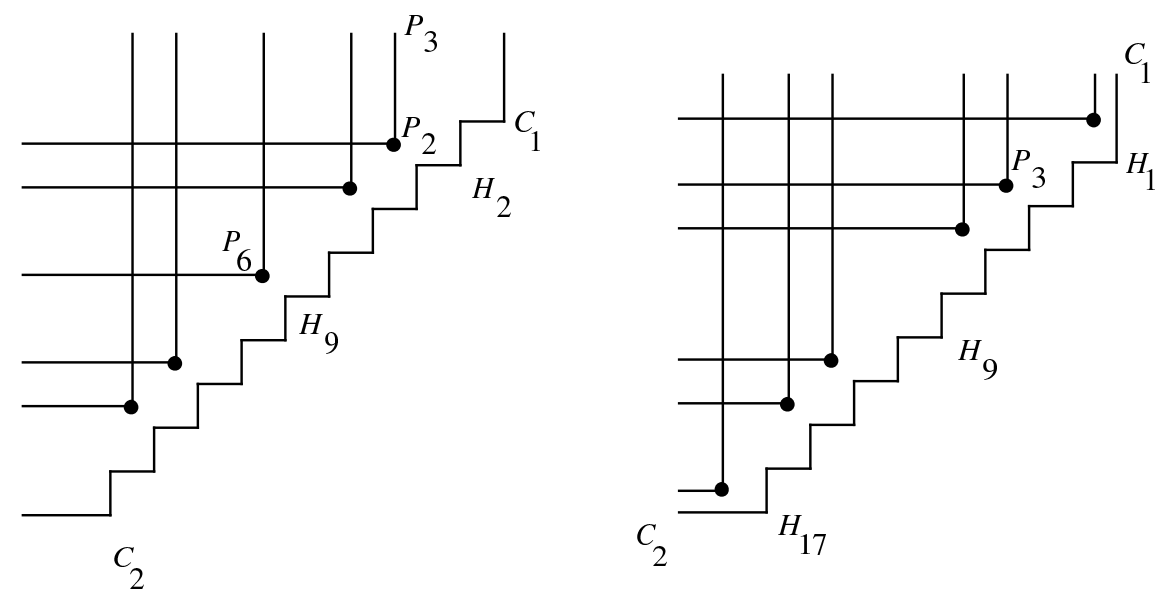

The $\mathrm{DH}_{5,8}$ orthodisks

The first step is to set up a space of complete geometric coordinates as we did for the $\mathrm{DH}_{1,1}$ surface. We face here a new difficulty: it is not the case that for every orthodisk $\Omega_{G d h}$ there is necessarily a conjugate orthodisk $\Omega_{G^{-1} d h}$, and vice versa. Thus, the geometric coordinate space has to be an appropriate subset of the natural parameter spaces of both orthodisks, and in addition we have to ensure that the coordinates are complete. We begin by an informal description with examples and then give a formal definition. After we give the formal definitions, we prove the setup suffices for our purposes in Lemmas 4.4.4 and 4.4.5.

The idea is to require that each branch point lie in a box which is bounded by (extensions of) suitable orthodisk edges. Not only will this allow for the existence of well-behaved moduli spaces, but it will also provide for important degenerations of the domain at the boundary.

In the following figures, these boxes are shaded in gray, and when necessary, separated by additional (suggestive) edges. When boxes overlap, they are shaded more darkly. We also discard from now on the fat dots marking the interior branch point - all interior vertices are branch points. 

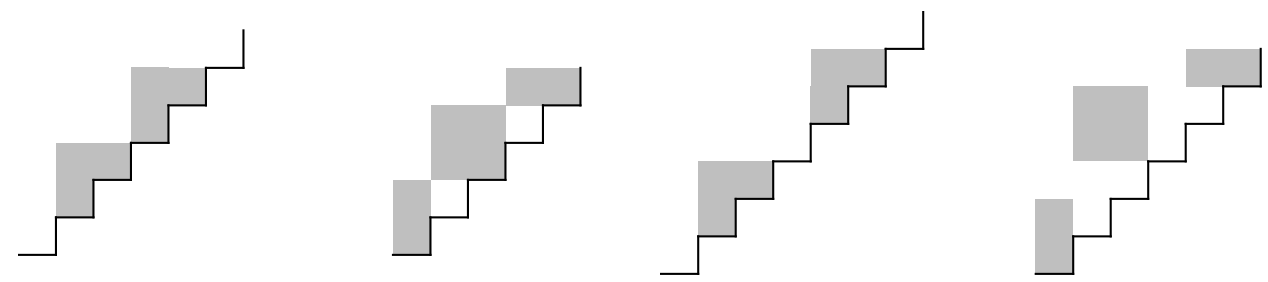

Bounding boxes for $\mathrm{DH}_{2,4}$ and $\mathrm{DH}_{2,5}$
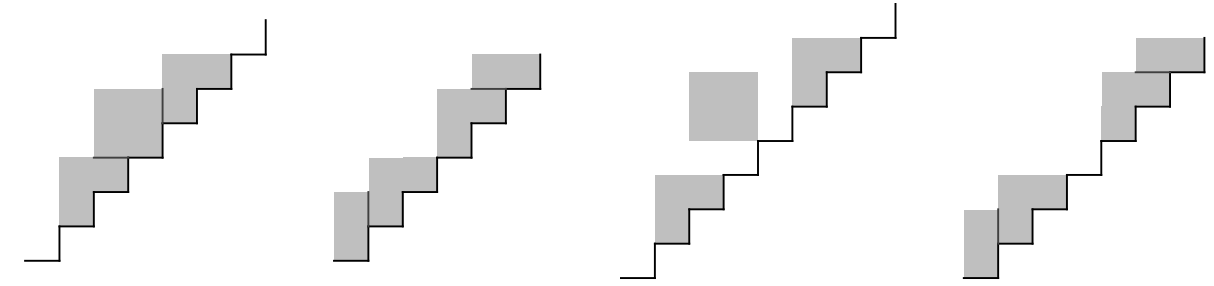

Bounding boxes for $\mathrm{DH}_{3,5}$ and $\mathrm{DH}_{3,6}$
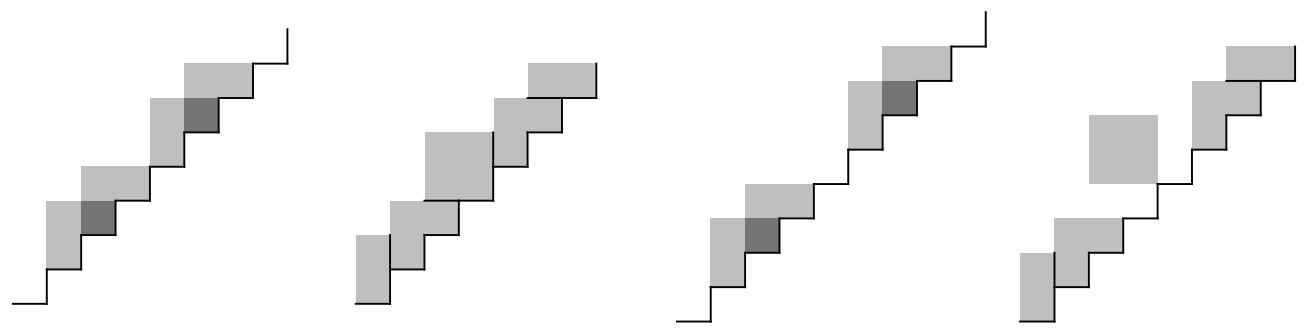

Bounding boxes for $\mathrm{DH}_{4,6}$ and $\mathrm{DH}_{4,7}$

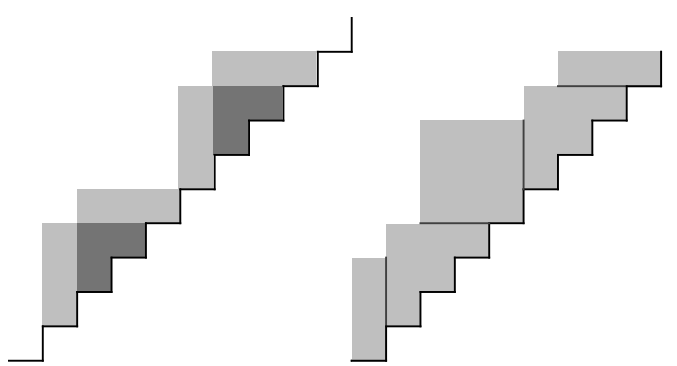

Bounding boxes for $\mathrm{DH}_{4,8}$

As a general rule, there are $m$ boxes for $\Omega_{G d h}$ (one for each branch point) which are intersections of finite rectangles with the orthodisks. For $\Omega_{G^{-1} d h}$, there are $m+1$ boxes, the two outermost of them being intersections of halfinfinite rectangular strips with the orthodisks. 
As an illustration, we explain in detail how the boxes can be deduced from the geometry for the $\mathrm{DH}_{2,4}$ surface:

In $\Omega_{G^{-1} d h}$, the edge $P_{4} P_{5}$ clearly has to lie above the edge $C_{2} H_{9}$. The conjugacy requirement for $\Omega_{G d h}$ forces that the edge $P_{4} P_{5}$ lie to the right of the edge $C_{2} H_{9}$. This forces, in $\Omega_{G d h}$, the edge $P_{3} P_{4}$ to lie above the edge $H_{8} H_{9}$. Observe that this is an inductive process to get border lines of the bounding boxes. In this most simple case, the induction immediately terminates: by symmetry, the branch point $P_{3}$ has to lie on the diagonal in $\Omega_{G^{-1} d h}$, hence the edge $P_{3} P_{4}$ lies left of $H_{4} H_{5}$, which causes, by conjugacy, the corresponding edges to lie below each other in $\Omega_{G d h}$. This way we get a backward induction in the general case, providing us with left and upper border lines for bounding boxes for all branch points on and below the diagonal, up to the outermost branch point in $\Omega_{G^{-1} d h}$ for which we do not get a left border line. By symmetry, we also obtain such borders for the branch points above the diagonal. Finally, the branch point on the diagonal is also bounded because the edge $P_{2} P_{3}$ lies left of $H_{1} H_{2}$ in $\Omega_{G d h}$ which gives a bound for $P_{3}$ in $\Omega_{G^{-1}} d h$.

The idea now is to specify the set of geometric coordinates as follows: First, record the edge lengths of the boundary of the outer sheet of the orthodisk $\Omega_{G d h}$. Then adjoin to those the positions of the branch points within the bounding boxes, measured as horizontal and vertical distances to the boundary.

In this $\mathrm{DH}_{2,4}$ example, the space of geometric coordinates can be described as the space of $\Omega_{G d h}$ (left) orthodisks with the requirement that the branch points lie inside the bounding boxes. Each such orthodisk is given by the data for its outer sheet boundary zigzag and the position of one point in the Lshaped box which itself is defined by the boundary zigzag. Using these data, it is possible to construct a unique conjugate orthodisk $\Omega_{G^{-1} d h}$ such that its branch points lie in the shaded bounding boxes. Furthermore, these coordinates are complete; any degeneration of the coordinates which does not come from an edge degeneration in $\Omega_{G d h}$ stems from one branch point coming close to the inner boundary of its shaded box. But this forces a branch point in $\Omega_{G^{-1} d h}$ to come close to the outer boundary, forcing a conformal degeneration. This concludes the discussion of the case $\mathrm{DH}_{2,4}$; the completeness of the coordinates for the general case of $\mathrm{DH}_{m, n}$ is proven in Lemma 4.4.5.

As a first step towards formally describing these boxes in the general case, we introduce families of cycles which will be useful both for specifying the coordinates and for defining the height function. In principle, there are four cases to distinguish depending on the parities of $m$ and $n$, but our notation will hide most of the differences.

Denote

$$
k=[m / 2] \quad \text { and } \quad d=\left[\frac{n-m}{2}\right]+1
$$


We first introduce cycles related to the outer sheet:

$$
\begin{aligned}
& \alpha_{1}: P_{1} C_{1} \quad \rightarrow \quad H_{1} H_{2} \\
& \alpha_{2}: C_{1} H_{1} \quad \rightarrow \quad H_{2} H_{3} \\
& \alpha_{3}: H_{1} H_{2} \quad \rightarrow \quad H_{3} H_{4} \\
& \ldots: \cdots \quad \rightarrow \quad \ldots \\
& \alpha_{2 n}: H_{2 n-2} H_{2 n-1} \rightarrow \quad H_{2 n} H_{2 n+1} \\
& \alpha_{2 n+1}: H_{2 n-1} H_{2 n} \quad \rightarrow \quad H_{2 n+1} C_{2} \\
& \alpha_{2 n+2}: H_{2 n} H_{2 n+1} \quad \rightarrow \quad P_{2 m+1} C_{2} \text {. }
\end{aligned}
$$

These cycles connect consecutive parallel edges of the outer sheet boundary. Their periods are realized as the finite edges. The symmetry condition on the orthodisk implies for these cycles

$$
\left|\operatorname{Per} \alpha_{k}\right|=\left|\operatorname{Per} \alpha_{2 n+3-k}\right| \text {. }
$$

Next we introduce four families of cycles called $\lambda_{k}, \rho_{k}, v_{k}$ and $\delta_{k}$ (for left, right, up, and down) connecting the horizontal and vertical edges of the inner sheets to certain carefully chosen horizontal and vertical edges of the outer sheet. The periods of these cycles will be used to define the bounding boxes:

$$
\begin{array}{lll}
\rho_{1}: P_{2} P_{3} & \rightarrow & H_{1} H_{2} \\
\rho_{2}: P_{4} P_{5} & \rightarrow & H_{3} H_{4} \\
\ldots: \cdots & \rightarrow \\
\rho_{k}: P_{2 k} P_{2 k+1} \rightarrow & H_{2 k-1} H_{2 k} \\
v_{1}: P_{1} P_{2} \rightarrow & H_{1} C_{1} \\
v_{2}: P_{3} P_{4} \rightarrow & H_{3} H_{2} \\
\ldots: \cdots & \cdots \\
v_{k}: P_{2 k-1} P_{2 k} \rightarrow & H_{2 k-1} H_{2 k-2} \\
\delta_{1}: P_{1} P_{2} \rightarrow & H_{d+3} H_{d+2} \\
\cdots: \cdots & \cdots \\
\delta_{k}: P_{2 k-1} P_{2 k} \rightarrow & H_{2 k+d+1} H_{2 k+d} \\
\lambda_{1}: P_{2} P_{3} \rightarrow & H_{d+3} H_{d+4} \\
\ldots: \cdots & \cdots \\
\lambda_{k}: P_{2 k} P_{2 k+1} \rightarrow & H_{2 k+d+1} H_{2 k+d+2} .
\end{array}
$$


In the case that $m$ is odd, we introduce in addition cycles for the box at $P_{k+1}$. For odd $n$ we set

$$
\begin{aligned}
\rho_{k+1}: P_{m+1} P_{m+2} \rightarrow & H_{n+1} H_{n+2} \\
\delta_{k+1}: P_{m} P_{m+1} \rightarrow & H_{n} H_{n+1} \\
v_{k+1}: P_{m} P_{m+1} \rightarrow & H_{n-d-2} H_{n-d-1} \\
\lambda_{k+1}: P_{m+1} P_{m+2} \rightarrow & H_{n+d+3} H_{n+d+4}
\end{aligned}
$$

and for even $n$ :

$$
\begin{aligned}
& \rho_{k+1}: P_{m+1} P_{m+2} \rightarrow H_{n} H_{n+1} \\
& \delta_{k+1}: P_{m} P_{m+1} \rightarrow H_{n+1} H_{n+2} \\
& v_{k+1}: P_{m} P_{m+1} \rightarrow H_{n-d-1} H_{n-d} \\
& \lambda_{k+1}: P_{m+1} P_{m+2} \rightarrow H_{n+d+2} H_{n+d+3} .
\end{aligned}
$$

This defines cycles emanating from the inner $\Omega_{G d h}$ sheet edges for the sheets containing the branch points $P_{2}, \ldots, P_{k}$, that is, for all sheets above the diagonal. For the sheets below the diagonal, we employ symmetry: Denote by $\alpha^{\prime}$ the image of a cycle $\alpha$ under reflection at the diagonal $y=-x$. We then define

$$
\begin{aligned}
\lambda_{j} & =v_{m+1-j}^{\prime} \\
\rho_{j} & =\delta_{m+1-j}^{\prime} \\
v_{j} & =\lambda_{m+1-j}^{\prime} \\
\delta_{j} & =\rho_{m+1-j}^{\prime} .
\end{aligned}
$$

This way all cycles are defined for $j=1, \ldots, m$.

The next two figures show all the cycles for $\mathrm{DH}_{5,8}$. For visibility, we have omitted both the names of the vertices and the fat dots indicating the branch points; we have also shortened the edges of the inner sheets.

The first figure shows the $v$-cycles and the $\delta$-cycles (thicker and shaded), the second the $\rho$-cycles and the $\lambda$-cycles (thicker and shaded). Informally, for each inner sheet of the orthodisk $\Omega_{G d h}$ there is a cycle of each type $(v, \delta, \rho, \lambda)$. The $v$-cycles have periods which point upward in $\Omega_{G d h}$ for the first half of the cycles in the upper-right part of the orthodisk, and similarly the periods of $\rho, \lambda, \delta$ point right, left and down. 


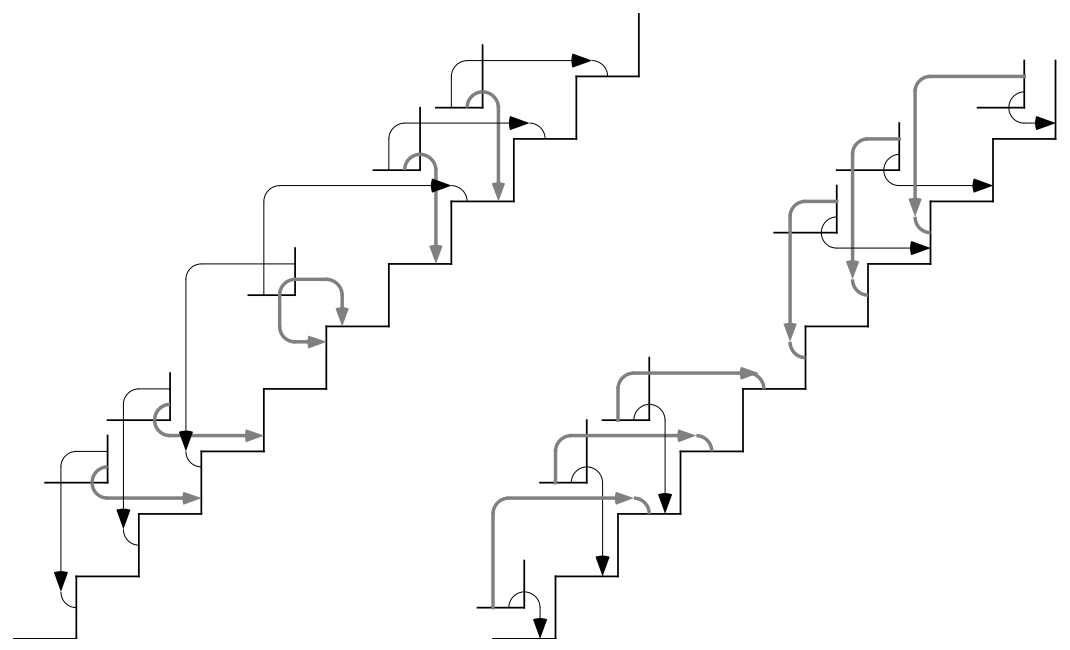

$v$-cycles and the $\delta$-cycles for $\mathrm{DH}_{5,8}$

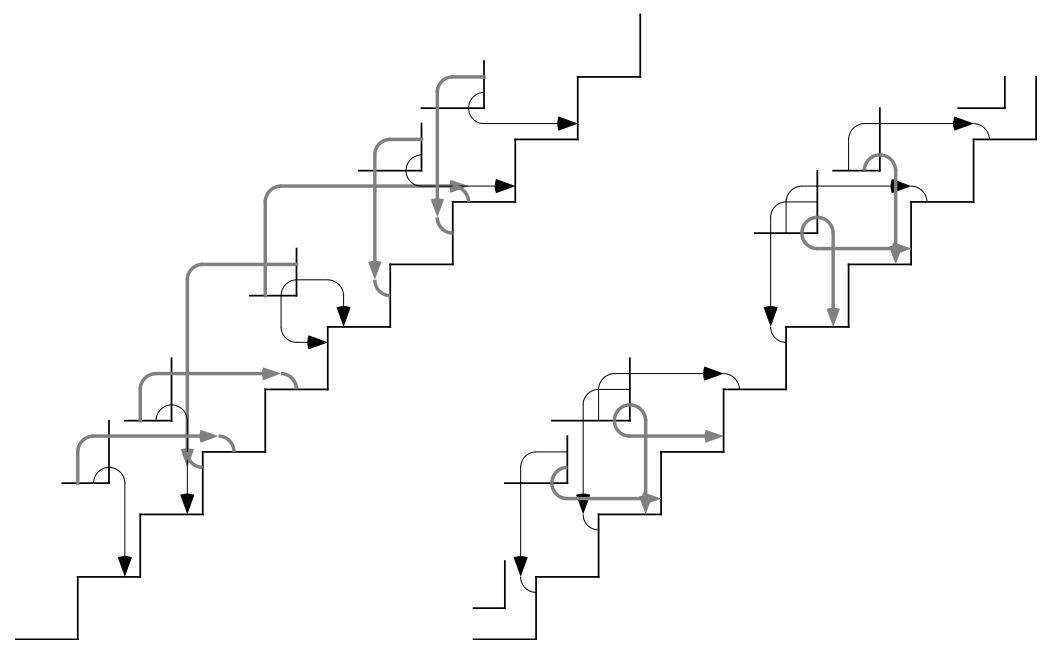

$\rho$-cycles and the $\lambda$-cycles for $\mathrm{DH}_{5,8}$

Now we are able to define the bounding boxes for the branch points:

Definition 4.4.1. Denote by $B_{2 j}$ the rectangle defined by

$$
\operatorname{Per} v_{j}>0 \text {, Per } \delta_{j}>0 \text {, Per } \lambda_{j}>0, \operatorname{Per} \rho_{j}>0
$$

and by $B_{2 j-1}$ the rectangle defined by

$$
\text { Per } \rho_{j-1}>0, \quad \text { Per } \delta_{j-1}>0, \quad \text { Per } \lambda_{j}>0, \quad \text { Per } v_{j}>0 .
$$

Note that the boxes $B_{1}$ and $B_{2 m+1}$ require the additional cycle $\lambda_{0}:=\alpha_{0}$ whereas $\rho_{0}$ is undefined so that the rectangle is open at one side. 
To define the geometric coordinates, we impose two restrictions on the branch points:

(1) $P_{j} \in B_{j}$

(2) $P_{j}$ must lie within the outer sheet.

While the second requirement is obviously necessary, the first will imply that the geometric coordinates are complete.

Definition 4.4.2. An orthodisk $\Omega_{G d h}$ or $\Omega_{G^{-1} d h}$ is called admissible if it satisfies the above condition for their respective branch points. A pair of orthodisks is called admissible if the orthodisks are conjugate and both are admissible.

Definition 4.4.3. The geometric coordinates of $\Omega_{G d h}$ are given by the periods of the cycles $\alpha_{j}, \rho_{j}, \lambda_{j}, v_{j}, \delta_{j}$ subject to the above condition. Similarly, the geometric coordinates of $\Omega_{G^{-1} d h}$ are given by the periods of these cycles subject to the above condition. This clearly defines two open subsets of a Euclidean space of dimension $g+1=m+n$. Each subset parametrizes the configuration space of admissible orthodisks $\Omega_{G d h}$ or $\Omega_{G^{-1} d h}$. Denote by $\Delta$ the intersection of these subsets; this set parametrizes admissible pairs of orthodisks and is called geometric coordinate space for $\mathrm{DH}_{m, n}$.

Lemma 4.4.4. $\Delta$ is an open cell of dimension $g-1$.

Proof. To prove openness we just observe that all imposed conditions are open in a certain linear space. To compute its dimension, we can easily get rid of the redundant equations of the definition of geometric coordinates for (say) $\Omega_{G d h}$ : The cycles $\delta_{j}-v_{j}$ and $\lambda_{i}-\rho_{i}$ are a certain sum of cycles $\alpha_{j}$. So to specify a symmetric orthodisk, we need just $m+n$ cycles. To prove that it is a nonempty cell, we construct a retraction to an interior point as follows: First, by using convex combinations of the outer sheet edge lengths, we can easily deform a given pair of admissible orthodisks to an admissible pair of orthodisks with the outer sheet periods being of length 1 . Now we move the edge $P_{2 m} P_{2 m+1}$ in $\Omega_{G^{-1} d h}$ down; this is unobstructed in $\Omega_{G^{-1}}$ dh but has to be accommodated by a movement of $P_{2 m} P_{2 m+1}$ to the left in $\Omega_{G d h}$. We move so far to the left here, that we are left of the edge $H_{2 n-1} H_{2 n-2}$. This allows us to move the edge $H_{2 m-1} H_{2 m}$ down in $\Omega_{G d h}$. We move so far down that we are, in $\Omega_{G^{-1} d h}$, left of the edge $\mathrm{H}_{2 n-2} H_{2 n-3}$, which enables us again to move $\mathrm{H}_{2 m-2} \mathrm{H}_{2 m-1}$ down. We continue this process, doing the same with the symmetric edges simultaneously. If we agree to move always to a position 0.5 apart from the left or lower boundary sheet, we will reach, in this way, a canonical point in the space $\Delta$. This defines the retraction. 
Lemma 4.4.5. $\Delta$ provides complete geometric coordinates.

Proof. We first have to show that each point in $\Delta$ gives a pair of welldefined symmetric (normalized) orthodisks. Using the coordinates of such a point, we see immediately that one can reconstruct the outer sheets for the $\Omega_{G d h}$ and the $\Omega_{G^{-1} d h}$ domains. Also, the positions of the branch points are specified so that they lie within the outer sheets and their respective bounding boxes. To obtain a correct pair of orthodisks, we have to make sure that these branch points do not coincide as one might expect for instance in the $\mathrm{DH}_{5,8}$ case where the bounding boxes overlap. However, this can be excluded as follows: If two branch points come close to each other, the periods of the two cycles connecting the parallel edges of their respective sheets vanish. This causes in the other domain a vanishing period between two inner sheets closer to the diagonal, and inductively we produce such a period degeneration on the diagonal, switching between the two domains. But on the diagonal, this cannot happen, because the branch point there is confined to the diagonal.

Finally, we have to show that when we have a sequence of admissible orthodisks leaving the space of geometric coordinates, at least one of the conformal structures of the $\Omega_{G d h}$ or $\Omega_{G^{-1} d h}$ orthodisks degenerates. The geometric degeneration means by definition that for at least one of the orthodisks, either an edge of the outer sheet boundary degenerates or a branch point hits the boundary of its bounding box. (The exceptional case of the half-infinite bounding rectangle in the domain $\Omega_{G^{-1} d h}$ domain does not actually allow the branch point to drift to infinity, because the corresponding period in the $\Omega_{G d h}$ domain is represented by a finite edge of the outer sheet, which is bounded by normalization.) In the first case, we get a node and are obviously done. In the second case, the branch point can either converge to some point on the outer boundary (in which case we pinch a cycle and are also done), or it can converge to a point on one of the virtual border lines of the bounding box. Then we clearly have no conformal degeneration for this orthodisk sequence, so we must prove that we get such a degeneration for the other family. (The next figure shows two conjugate orthodisks with bounding boxes. Equally labeled arrows indicate equal distances from orthodisk edges to the virtual border lines of bounding boxes. As long as these distances are equal, the branched point can move freely within their bounding boxes.)

Suppose, for concreteness, that we have a sequence of orthodisks $\Omega_{G d h}(n)$ where a branch point converges to an upper virtual border line of its bounding box. This branch point $P_{k}$ belongs to an edge $E=P_{k} P_{k-1}$ which comes close to this virtual line from below. By conjugacy, in the corresponding $\Omega_{G^{-1}} d h$ orthodisks, the corresponding edge $E^{*}=P_{k} P_{k-1}$ comes close to a bounding border line to the right. The other endpoint $P_{k-1}$ of this edge is a branch point 
for $\Omega_{G^{-1} d h}$ which hence comes arbitrarily close to the outer sheet boundary. So we pinch a curve in $\Omega_{G^{-1}} d h$ and get a conformal degeneration. The other cases are treated similarly.
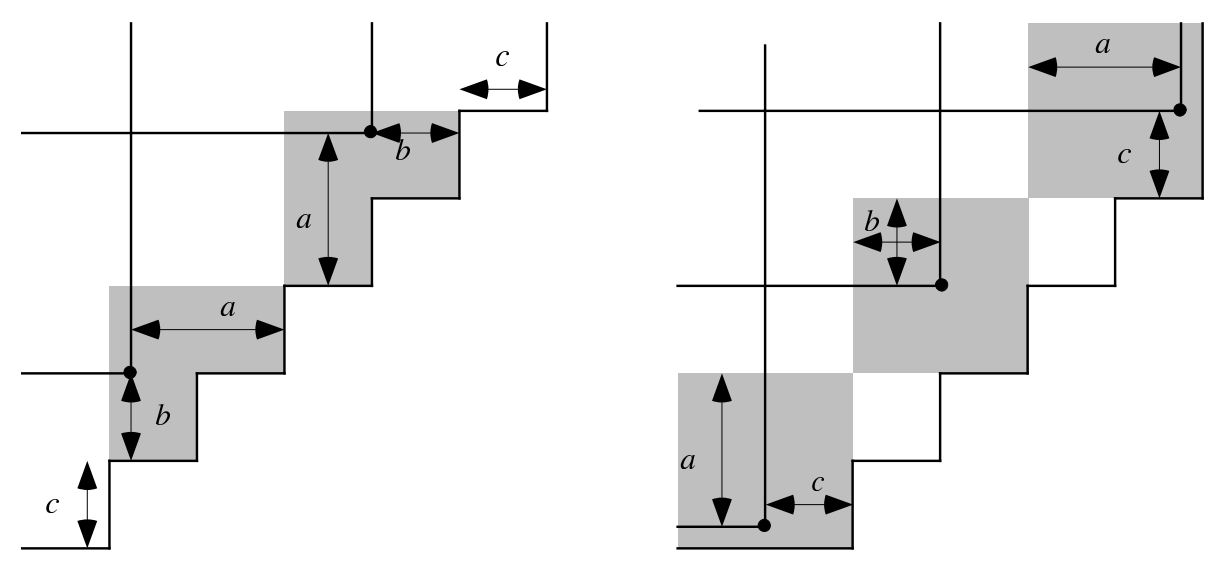

Conjugate orthodisks with coupled branch points

4.5. Height functions for $\mathrm{DH}_{m, n}$. In this section, we will provide a proper height function defined on the geometric coordinate space $\Delta$ of the previous section for the $\mathrm{DH}_{m, n}$ surfaces with $m+n>2$.

We begin by stating the basic requirements on a height function which we require for the steps of the proof of Theorem $\mathrm{B}(\mathrm{i})$ in $\S \S 4-6$.

To estimate the rate of growth/decay of extremal lengths in terms of geometric degenerations, we need an asymptotic expression for the extremal lengths of the cycles we use in the height function. Such an expression (see [Oht]) is only known to us in the case that the cycle is a lift of an arc in the upper half-plane connecting two disjoint edges. This is the case of a conformal quadrilateral. Informally, an admissible cycle in an orthodisk is one which can be reduced to such a cycle as just described. More precisely, we recall from $\S 4.1$ the

Definition 4.5.1. A cycle in an orthodisk is called admissible if it is either simple and connects a pair of symmetric edges or has two symmetric components which do not cross the diagonal.

Using admissible cycles, we will compose the height as a sum of the following terms:

Definition 4.5.2. Let $c$ be an admissible cycle. Define

$$
\mathcal{H}(c)=\left|e^{1 / \operatorname{Ext}_{\Omega_{G d h}}(c)}-e^{1 / \operatorname{Ext}_{\Omega_{G}-1}(c)}\right|^{2}+\left|e^{\operatorname{Ext}_{\Omega_{G d h}}(c)}-e^{\operatorname{Ext}_{\Omega_{G^{-1}}}(c)}\right|^{2} .
$$


Recall that the conformal polygon of a $\mathrm{DH}_{m, n}$-surface is (two-fold) symmetric and has $2 m+2 n+4$ vertices; there are thus $m+n$ conformal moduli of such a shape.

In addition to the requirement that the cycles detect conformal degenerations, we will also need some more properties which we will state here but only explain and use in $\S 6$. (There, we take a low genus reflexive orthodisk, and append some thrice punctured spheres to it along nodes; we then open the nodes to obtain high genus surfaces in a stratum $\mathcal{Y} \subset \Delta$ along which we flow in $\S 5$ to a reflexive orthodisk solution to our problem.) This 'regeneration' in $\S 6$ requires a set of $m+n$ cycles for the height which provide conformal coordinates for the points in $\Delta$; moreover, the set of curve systems, specified for the higher genus surface, which do not degenerate as we pinch the surface to a noded surface (i.e., are either not pinched into the node or do not cross the node) should also provide local coordinates for the boundary stratum of lower genus surfaces.

In particular, there should be exactly two 'bad' curves (which degenerate as $\mathrm{DH}_{n+1, n+1}$ degenerates to $\mathrm{DH}_{n, n}$, i.e., with extremal lengths which go to 0 or $\infty$ as $P_{1}$ and $H_{1}$ converge) in the $n=m$ case, and one bad curve in the $n \neq m$ case (i.e., which degenerates as $m+1 \rightarrow m$ in $\mathrm{DH}_{m+1, n}$ ) with an extremal length that goes to $\infty$ as the finite vertex nearest the central vertex approaches it. Also, as we shall see in $\S 5$, the technical requirements of the Noncritical Flow Step will restrict the edges which are allowed for the feet of the bad curves; we postpone the discussion of this until after Proposition 5.3.2 in $\S 5.3$.)

For the definition of the height function, we distinguish two cases: This distinction comes from the inductive design of the proofs of Theorem A and B: In the case $C T_{n}=\mathrm{DH}_{n, n}$, we use the edge $C_{1} H_{1}$ for pushing, and this requires the cycles to be chosen so that no cycle foots on this edge and precisely one cycle encircles it. In the case that we want to prove existence of a $\mathrm{DH}_{m, n}$ with $m<n$, we need the central edges of the outer sheet boundary zigzag for pushing.

Besides that, the choice of the cycles for the height function follows the same general rules: By using cycles which connect infinite edges with infinite edges, we detect degeneracies of inner sheets, and by using cycles which encircle or foot on outer sheet edges, we enforce properness using the monodromy lemma.

Finally, as a general rule, all our cycles will be symmetric with respect to the diagonal.

(1) $\mu$ connects $C_{1} P_{1}$ with $C_{2} P_{2 m+1}$.

(2) $\nu$ connects $C_{1} P_{1}$ with $H_{1} H_{2}$ and $C_{2} P_{2 m+1}$ with $H_{2 n+1} H_{2 n}$. 
(3) $\rho$ connects $H_{n} H_{n+1}$ with $P_{m-1} P_{m}$ and $H_{n+1} H_{n+2}$ with $P_{m+1} P_{m+2}$.

(4) $\sigma$ connects $H_{1} C_{1}$ to $P_{1} P_{2}$ and $H_{2 n+1} C_{2}$ to $P_{2 m} P_{2 m+1}$.

(5) $\delta$ connects $P_{1} P_{2}$ to $H_{n} H_{n+1}$ and $P_{2 m} P_{2 m+1}$ to $H_{n+2} H_{n+3}$.

(6) $\gamma$ connects $H_{n-1} H_{n}$ to $H_{n+2} H_{n+3}$.

(7) $\beta_{k}$ connects $P_{1} P_{2}$ with $P_{2 k-1} P_{2 k}$ and $P_{2 m} P_{2 m+1}$ with $P_{2 m+2-k} P_{2 m+1-k}$.

(8) $\alpha_{k}$ encircles $H_{k} H_{k+1}$ and $H_{2 n+2-k} H_{2 n+1-k}$.

(9) $\tau$ connects $P_{1} P_{2}$ with $P_{2 m} P_{2 m+1}$.

The cycle $\rho$ has been chosen to replace $\alpha_{n-1}$ which would interfere with the pushing edge $H_{1} H_{2}$ for small $n$.

Now we define the cycles for the height function:

In the case $m=n=1$, select the cycles $\mu$ and $\nu$. In the case $m=$ $n>1$, select the cycles $\mu, \nu, \gamma, \delta, \beta_{2}, \ldots, \beta_{m-1}, \alpha_{2}, \ldots, \alpha_{n-1}$. In the case $m=1<n$, select $\mu, \rho, \sigma, \alpha_{1}, \ldots, \alpha_{n-2}$ and in the case $1<m<n$ select $\mu, \rho, \sigma, \tau, \alpha_{1}, \ldots, \alpha_{n-2}, \beta_{2}, \ldots, \beta_{m-1}$.

Note that if $n=2$ there are no $\alpha$-cycles. In all cases, we have selected $m+n$ cycles.

Observe that for $m=n=1$, the cycle $\mu$ becomes mauve and $\nu$ becomes blue so that the height defined here coincides with the one defined in $\S 4.3$ for $\mathrm{DH}_{1,1}$.

Definition 4.5.3. The height for the $\mathrm{DH}_{m, n}$ surface is defined as the sum of the heights of all selected cycles for the values of $(m, n)$.

Example 4.5.4. As an example, we show the cycles for the height of the $\mathrm{DH}_{2,4}$ and the $\mathrm{DH}_{3,3}$ surface which are relevant for the properness proof:

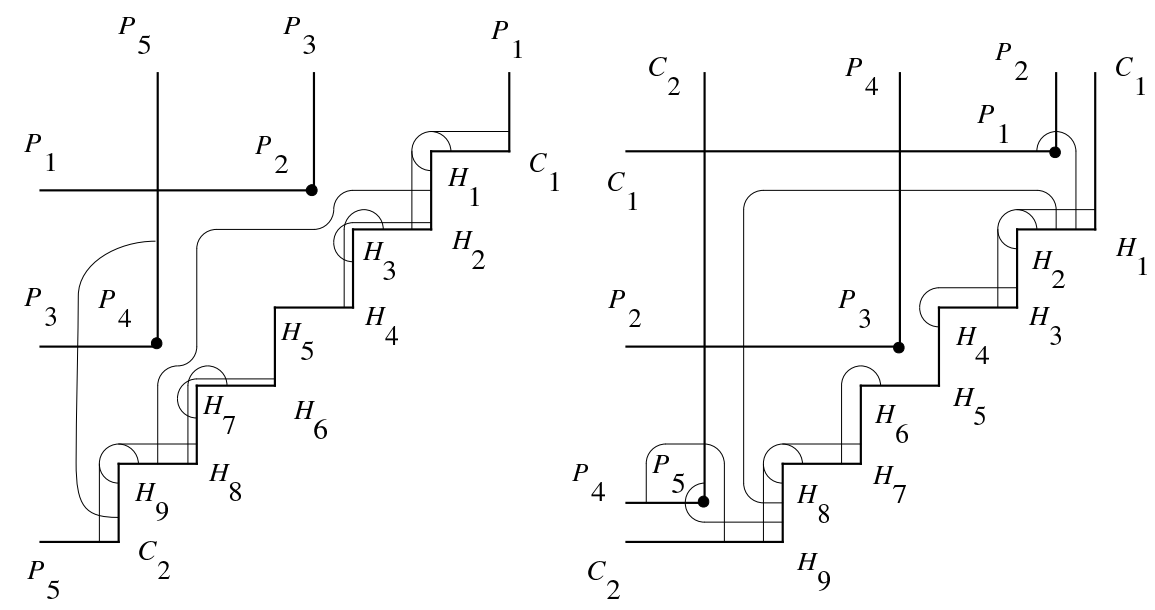

The cycles for the height function for $\mathrm{DH}_{2,4}$ 

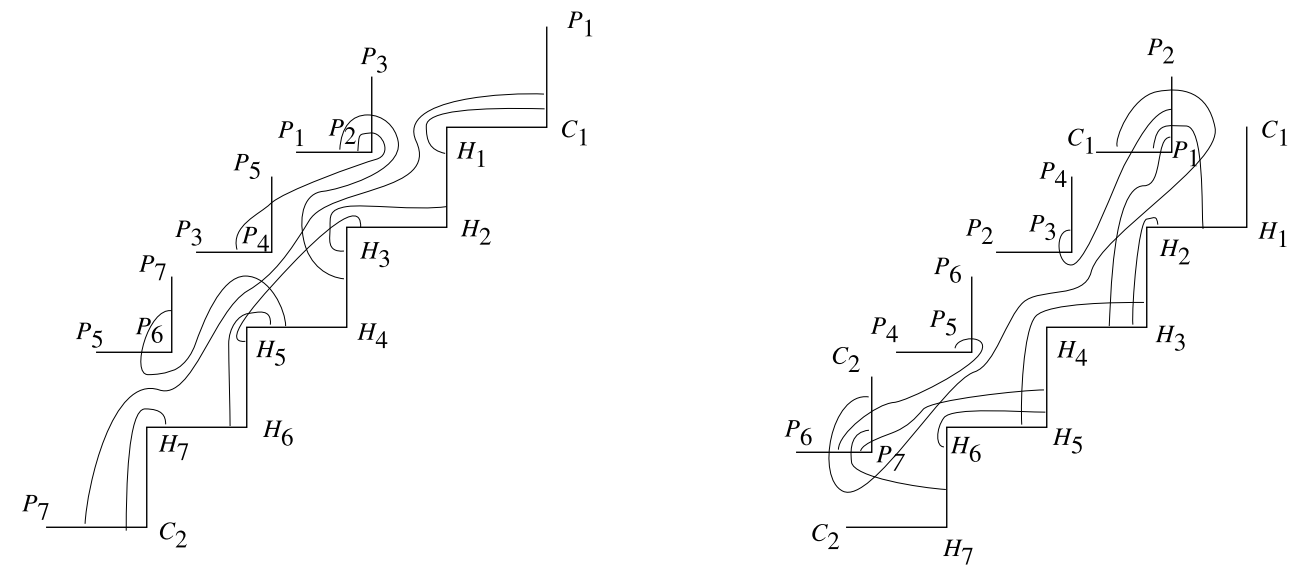

The cycles for the height function for $\mathrm{DH}_{3,3}$

We record for later reference the following trivial consequence of our using curves in the height function whose extremal lengths are conformal coordinates for the domains defined by the geometric coordinates.

Lemma 4.5.5. Let $X=\left\{\Omega_{G d h}, \Omega_{G^{-1} d h}\right\}$ be an orthodisk system in $\Delta$. Then $X$ is reflexive if and only if $\mathcal{H}(X)=0$.

4.6. Properness of the height functions for $\mathrm{DH}_{m, n}$. In this section, we prove, modulo the postponed monodromy argument.

THEOREM 4.6.1. The height functions $\mathcal{H}$ from $\S 4.5$ are proper.

Proof. To show that the height functions from $\S 4.5$ are proper, we need to prove that for any sequence of points in $\Delta$ converging to some boundary point, the height goes to infinity. First, by Lemma 4.4.5, at least one of the two orthodisks degenerates conformally. We will now analyze the possible geometric degenerations.

For this, we normalize the orthodisks in the sequence so that the finite edges of the outer boundary of $\Omega_{G d h}$ has total length 1 . Such a scaling does not affect the conformal structure.

Suppose first that the degeneration occurs with an inner sheet branch point. As proven in Lemma 4.4.5, this implies that some branch point(s) converges to a point on the outer sheet boundary. Consider the inner sheet with the smallest $k$ so that $P_{k}$ is a branch point of that sheet, for which this degeneration happens. 
If $k=1$ and the edge $H_{1} C_{1}$ in $\Omega_{G d h}$ does not shrink to 0, then Ext $\sigma \rightarrow 0$ in $\Omega_{G^{-1} d h}$ for $m<n$ and Ext $\nu \rightarrow 0$ in $\Omega_{G^{-1} d h}$, but the corresponding extremal length remains positive in $\Omega_{G d h}$. If the edge $H_{1} C_{1}$ in $\Omega_{G d h}$ does shrink to 0 , the monodromy argument can be applied to $\alpha_{1}(m<n)$ or to $\nu(m=n)$.

If $k>1$, the sheet containing $P_{k}$ is separated from the outer sheet boundary by a $\beta$-cycle or (for a central inner sheet by the cycle $\mu$ ) which will be pinched in one of the orthodisks but not the other, and which also will not be pinched by other inner sheets degenerating. Hence the height of this cycle will go to $\infty$.

Now let us assume that all inner sheets are bounded away from the outer sheet boundary and that the geometric degeneration occurs only in the outer boundary sheet of $\Omega_{G d h}$.

We begin by analyzing one execeptional case: Suppose that the edge $C_{1} H_{1}$ in $\Omega_{G d h}$ shrinks to 0 . The monodromy lemma, applied to the cycle $\alpha_{1}$ (in the $m<n$ case) or the cycle $\nu$ (in the $n=m$ case) proves the properness of the height function of this cycle.

Now suppose that the edge $C_{1} H_{1}$ does not shrink to 0 , and consider the smallest $k$ for which the edge $H_{k} H_{k+1}$ shrinks to 0 . The monodromy lemma can be applied to a neighboring cycle $\alpha_{k}$ or $\alpha_{k \pm 1}$ This treats all $k$ but $k=n$ in the case $m<n$ and $k=1$ in the case $m=n=2$.

Consider the case $m<n$ and suppose that the central edge $H_{n} H_{n+1}$ shrinks to 0 . This is the only geometric degeneration occurring in the orthodisk. Hence we can apply the monodromy lemma to $\rho$.

Consider the case $m=n=2$ and suppose that the finite edge $H_{1} H_{2}$ shrinks to 0 . This is again the only geometric degeneration occurring in the orthodisk. Hence we can apply the monodromy lemma to $\nu$.

This treats all possible cases, and the theorem is proven.

4.7. A monodromy argument. In this section, we prove that the periods of orthodisks have incompatible logarithmic singularities in suitable coordinates and apply this to prove the monodromy theorem 4.1.2. The main idea has already been used in [WW]: to study the dependence of extremal lengths of the geometric coordinates, it is necessary to understand the asymptotic dependence of extremal lengths of the degenerating conformal polygons (which is classical and well-known, see [Oht]), and the asymptotic dependence of the geometric coordinates of the degenerating conformal polygons. This dependence is given by Schwarz-Christoffel maps which are well-studied in many special cases. Especially, it is known that these maps possess asymptotic expansions in logarithmic terms. Instead of computing this expansion explicitly for the two maps needed (which is possible but tedious), we use a monodromy argument to show that the crucial logarithmic terms have a different sign for the two expansions. 
Let $\Delta$ be a domain of dimension at least two defining geometric coordinates for a pair of orthodisks $X_{1}$ and $X_{2}$ corresponding to given formal Weierstrass data as usual. Suppose $\gamma$ is a cycle in the underlying conformal polygon which joins edges $P_{1} P_{2}$ and $Q_{1} Q_{2}$ which are parallel (and hence nonadjacent) in the orthodisks. In our applications, $\gamma$ will be one of the $\alpha$-cycles used in the height function. Denote by $R_{1}$ the vertex before $Q_{1}$ and by $R_{2}$ the vertex after $Q_{2}$ and observe that by assumption, $R_{2} \neq P_{1}$ and $P_{2} \neq R_{1}$. Introduce a second cycle $\beta$ which connects $R_{1} Q_{1}$ with $Q_{2} R_{2}$.

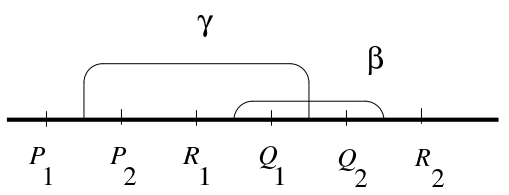

Monodromy argument

We formulate the claim of Theorem 4.1.2 more precisely in the following two lemmas:

LEMma 4.7.1. Suppose that for a sequence $p_{n} \in \Delta$ with $p_{n} \rightarrow p_{0} \in \partial \Delta$, $\operatorname{Ext}_{X_{1}\left(p_{n}\right)}(\gamma) \rightarrow 0$ and $\operatorname{Ext}_{X_{2}\left(p_{n}\right)}(\gamma) \rightarrow 0$. Suppose furthermore that $\gamma$ is a cycle encircling a single edge which degenerates geometrically to 0 as $n \rightarrow \infty$ in $X_{1}\left(p_{n}\right)$. Then

$$
\left|e^{1 / \operatorname{Ext}_{X_{1}\left(p_{n}\right)}(\gamma)}-e^{1 / \operatorname{Ext}_{X_{2}\left(p_{n}\right)}(\gamma)}\right|^{2} \rightarrow \infty
$$

Lemma 4.7.2. Suppose that for a sequence $p_{n} \in \Delta$ with $p_{n} \rightarrow p_{0} \in \partial \Delta$ we have that $\operatorname{Ext}_{X_{1}\left(p_{n}\right)}(\gamma) \rightarrow \infty$ and $\operatorname{Ext}_{X_{2}\left(p_{n}\right)}(\gamma) \rightarrow \infty$. Suppose furthermore that $\gamma$ is a cycle with an endpoint on an edge which degenerates geometrically to 0 as $n \rightarrow \infty$ in $X_{1}\left(p_{n}\right)$. Then

$$
\left|e^{\operatorname{Ext}_{X_{1}\left(p_{n}\right)}(\gamma)}-e^{\operatorname{Ext}_{X_{2}\left(p_{n}\right)}(\gamma)}\right|^{2} \rightarrow \infty .
$$

Proof. We first prove Lemma 4.7.1.

Consider the conformal polygons corresponding to the pair of orthodisks. Normalize the punctures by Möbius transformations so that

$$
P_{1}=-\infty, \quad P_{2}=0, Q_{1}=\varepsilon, \quad Q_{2}=1
$$

for $X_{1}$ and

$$
P_{1}=-\infty, \quad P_{2}=0, Q_{1}=\varepsilon^{\prime}, \quad Q_{2}=1
$$

for $X_{2}$. By the assumption of Lemma 4.7.1, we know that $\varepsilon, \varepsilon^{\prime} \rightarrow 0$ as $n \rightarrow \infty$. We now apply the monodromy Corollary 4.7 .5 below to the curve $\varepsilon_{0} e^{i t}$ and 
conclude that either

$$
\frac{\left|\operatorname{Per} \beta\left(X_{1}\right)\right|}{\left|\operatorname{Per} \gamma\left(X_{1}\right)\right|}+\frac{1}{\pi} \log \varepsilon
$$

is single-valued in $\varepsilon$ while

$$
\frac{\left|\operatorname{Per} \beta\left(X_{2}\right)\right|}{\left|\operatorname{Per} \gamma\left(X_{2}\right)\right|}-\frac{1}{\pi} \log \varepsilon^{\prime}
$$

is single-valued in $\varepsilon^{\prime}$, or the same statement holds for analogous quantities with opposite signs. Without loss of generality we can treat the first case.

Now suppose that $\varepsilon^{\prime}$ is real analytic (and hence single-valued) in $\varepsilon$ near $\varepsilon=0$. Then use of the fact that $X_{1}$ and $X_{2}$ are conjugate implies that the absolute lengths of $\beta$ in $X_{1}$ and $X_{2}$ are equal, as are those of $\gamma$; hence

$$
\frac{\left|\operatorname{Per} \beta\left(X_{1}\right)\right|}{\left|\operatorname{Per} \gamma\left(X_{1}\right)\right|}=\frac{\left|\operatorname{Per} \beta\left(X_{2}\right)\right|}{\left|\operatorname{Per} \gamma\left(X_{2}\right)\right|} \text {. }
$$

Thus we see that, after subtracting (4.7.1b) from (4.7.1a),

$$
\log \left(\varepsilon \varepsilon^{\prime}(\varepsilon)\right)
$$

is single-valued in $\varepsilon$ near $\varepsilon=0$ which contradicts that $\varepsilon, \varepsilon^{\prime} \rightarrow 0$.

Now Ohtsuka's extremal length formula states that for the current normalization of $X_{1}\left(p_{n}\right)$, we have

$$
\operatorname{Ext}(\gamma)=O\left(|\log \varepsilon|^{-1}\right)
$$

(see Lemma 4.5.3 in [WW] and [Oht]). We conclude that

$$
\left|e^{1 / \operatorname{Ext}_{X_{1}\left(p_{n}\right)}(\gamma)}-e^{1 / \operatorname{Ext}_{X_{2}\left(p_{n}\right)}(\gamma)}\right|=O\left(\frac{1}{\varepsilon}-\frac{1}{\varepsilon^{\prime}}\right)
$$

which goes to infinity, since we have shown that $\varepsilon$ and $\varepsilon^{\prime}$ tend to zero at different rates. This proves Lemma 4.7.1.

The proof of Lemma 4.7.2 is very similar: For convenience, we normalize the points of the punctured disks such that

$$
P_{1}=-\infty, \quad P_{2}=0, Q_{1}=1, \quad Q_{2}=1+\varepsilon
$$

for $X_{1}$ and

$$
P_{1}=-\infty, \quad P_{2}=0, Q_{1}=1, \quad Q_{2}=1+\varepsilon^{\prime}
$$

for $X_{2}$.

By the assumption of Lemma 4.7.2, we know that $\varepsilon, \varepsilon^{\prime} \rightarrow 0$ as $n \rightarrow \infty$. We now apply the monodromy Corollary 4.7 .5 below to the curve $1+\varepsilon_{0} e^{i t}$ and conclude that

$$
\frac{\operatorname{Per} \gamma\left(X_{1}\right)}{\operatorname{Per} \beta\left(X_{1}\right)}+\frac{1}{\pi} \log \varepsilon
$$


is single-valued in $\varepsilon$ while

$$
\frac{\operatorname{Per} \gamma\left(X_{2}\right)}{\operatorname{Per} \beta\left(X_{2}\right)}-\frac{1}{\pi} \log \varepsilon^{\prime}
$$

is single-valued in $\varepsilon^{\prime}$. The rest of the proof is identical to the proof of Lemma 4.7.1.

To prove the needed Corollary 4.7.5, we need asymptotic expansions of the extremal length in terms of the geometric coordinates of the orthodisks. Though not much is known explicitly about extremal lengths in general, for the chosen cycles we can reduce this problem to an asymptotic control of SchwarzChristoffel integrals. Their monodromy properties allow us to distinguish their asymptotic behavior by the sign of logarithmic terms.

We introduce some notation: Suppose we have an orthodisk such that the angles at the vertices alternate between $\pi / 2$ and $-\pi / 2$ modulo $2 \pi$. Consider the Schwarz-Christoffel map

$$
F: z \mapsto \int_{i}^{z}\left(t-t_{1}\right)^{a_{1} / 2} \cdot \ldots \cdot\left(t-t_{n}\right)^{a_{n} / 2}
$$

(see §3.2) from a conformal polygon with vertices at $t_{i}$ to this orthodisk. Choose four distinct vertices $t_{i}, t_{i+1}, t_{j}, t_{j+1}$ so that $j \equiv i(\bmod 2)$, ensuring that the edges $t_{i} t_{i+1}$ and $t_{j} t_{j+1}$ are parallel in the orthodisk geometry. (See the figure below.) Introduce a cycle $\gamma$ in the upper half-plane connecting edge $\left(t_{i}, t_{i+1}\right)$ with edge $\left(t_{j}, t_{j+1}\right)$ and denote by $\bar{\gamma}$ the closed cycle obtained from $\gamma$ and its mirror image across the real axis. Similarly, denote by $\beta$ the cycle connecting $\left(t_{j-1} t_{j}\right)$ with $\left(t_{j+1} t_{j+2}\right)$ and by $\bar{\beta}$ the cycle together with its mirror image.

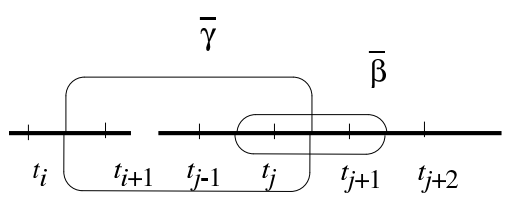

Cycles for analytic continuation

Now consider the Schwarz-Christoffel period integrals

$$
\begin{aligned}
& F(\gamma)=\frac{1}{2} \int_{\gamma}\left(t-t_{1}\right)^{a_{1} / 2} \cdot \ldots \cdot\left(t-t_{n}\right)^{a_{n} / 2}, \\
& F(\beta)=\frac{1}{2} \int_{\beta}\left(t-t_{1}\right)^{a_{1} / 2} \cdot \ldots \cdot\left(t-t_{n}\right)^{a_{n} / 2},
\end{aligned}
$$

as multi-valued functions depending on the now complex parameters $t_{i}$. 
LEMMA 4.7.3. Under analytic continuation of $t_{j+1}$ around $t_{j}$ the periods change their values as shown:

$$
\begin{aligned}
& F(\gamma) \rightarrow F(\gamma)+2 F(\beta), \\
& F(\beta) \rightarrow F(\beta)
\end{aligned}
$$

Proof. The proof is the same as [WW, Lemma 4.4.1]: the path of analytic continuation of $t_{j+1}$ around $t_{j}$ gives rise to an isotopy of $\mathbb{C}$ which moves $t_{j+1}$ along this path. This isotopy drags $\beta$ and $\gamma$ to new cycles $\beta^{\prime}$ and $\gamma^{\prime}$.

Because the curve $\beta$ is defined to surround $t_{j}$ and $t_{j+1}$, the analytic continuation merely returns $\beta$ to $\beta^{\prime}$. Thus, because $\beta^{\prime}$ equals $\beta$, their periods are also equal. On the other hand, the curve $\gamma$ is not equal to the new 'dragged' curve $\gamma^{\prime}$. To see this, note that the period of $\gamma^{\prime}$ is obtained by developing the flat structure of the doubled orthodisk along $\gamma^{\prime}$. To compute this flat structure, observe the crucial fact that the angles at the orthodisk vertices are either $\pi / 2$ or $-\pi / 2$, modulo $2 \pi$; thus the angles of the doubled orthodisk equal $\pi$, modulo $2 \pi$. Thus the arc $\gamma^{\prime}$ develops into the union of the $\operatorname{arc} \gamma$ with the $\operatorname{arcs} \beta$ and $\bar{\beta}$; in particular, we see that the period of $\gamma^{\prime}$ equals the period of $\gamma$ plus twice the period of $\beta$.

Now denote $\delta:=t_{j+1}-t_{j}$ and fix all $t_{i}$ other than $t_{j+1}$, which we regard as the independent variable.

Corollary 4.7.4. The function $F(\gamma)-\frac{\log \delta}{\pi i} F(\beta)$ is single-valued and holomorphic near $\delta=0$.

Proof. By definition, the function is locally holomorphic near $\delta=0$. By Lemma 4.7.3 it is single-valued.

Now, for the properness argument, we are interested in the geometric coordinates - these are the absolute values of the periods. More precisely we are interested in

$$
\|F(\gamma)\|:=|\operatorname{Re} F(\gamma)|+|\operatorname{Im} F(\gamma)| .
$$

We translate the above statement about periods into a statement about their respective absolute values.

We consider two conjugate orthodisks parametrized by Schwarz-Christoffel maps $F_{1}$ and $F_{2}$ defined on the same conformal polygon. Recall that we have constructed $\beta$ and $\gamma$ to be purely horizontal or vertical.

Corollary 4.7.5. Either $\left\|F_{1}(\gamma)\right\|-\frac{\log \delta}{\pi}\left\|F_{1}(\beta)\right\|$ or $\left\|F_{1}(\gamma)\right\|+\frac{\log \delta}{\pi}\left\|F_{1}(\beta)\right\|$ is real analytic in $\delta$ for $\delta=0$. In the first case, $\left\|F_{2}(\gamma)\right\|+\frac{\log \delta}{\pi}\left\|F_{2}(\beta)\right\|$ is real analytic in $\delta$, and in the second $\left\|F_{2}(\gamma)\right\|-\frac{\log \delta}{\pi}\left\|F_{1}(\beta)\right\|$ is. 
Proof. Recall from the tedious $\S 4.1$ that the above periods are linear combinations of the geometric coordinates where the coefficients are just signs, in the sense of being elements of $\{1,-1, i,-i\}$. Now by construction, $F_{j}(\beta)$ is purely real or purely imaginary; moreover, the direction of $F_{j}(\beta)$ is $\pm i$ times the direction of $F_{j}(\gamma)$. This, together with Corollary 4.7.4, implies the first claim. Next, note that (see for example the table in §3.4) if we turn left at a vertex in $X_{1}$, we will turn right at the corresponding vertex in the conjugate orthodisk $X_{2}$, and vice versa. Thus, if the directions of the corresponding edges for $\gamma$ in corresponding orthodisks differ by $+i$, then the directions of the corresponding edges for $\beta$ will differ by a $-i$, and vice versa. This implies the second claim.

\section{The gradient flow}

\subsection{Overall strategy.}

5.1.1. In this section we continue the proof of the existence portions of the main theorems, Theorem $\mathrm{A}$ and Theorem $\mathrm{B}(\mathrm{i})$. In the previous sections, we assigned to a configuration $\mathcal{C}$ (the ones $\mathcal{C}=(U D)^{k} U$ and $\mathcal{C}=\mathrm{DH}_{m, n}$ were of principal interest) a moduli space $\Delta=\Delta_{\mathcal{C}}$ of pairs of conformal structures $\left\{\Omega_{G d h}, \Omega_{G^{-1} d h}\right\}$ equipped with geometric coordinates $\overrightarrow{\mathbf{t}}=\left(t_{i}, \ldots, t_{l}\right)$.

In the last section, we defined a height function $\mathcal{H}$ on the moduli space $\Delta$ and proved that it was a proper function: as a result, there is a critical point for the height function in $\Delta$, and our overall goal in the next pair of sections is a proof that one of these critical points represents a reflexive orthodisk system in $\Delta$, and hence, by Theorem 3.3.5, a minimal surface of the configuration $\mathcal{C}$. Our goal in the present section is a description of the tangent space to the moduli space $\Delta$ : we wish to display how infinitesimal changes in the geometric

coordinates $\overrightarrow{\mathbf{t}}$ affect the height function. In particular, it would certainly be sufficient for our purposes to prove the statement:

MODEL 5.1.1. If $\overrightarrow{\mathbf{t}}_{0}$ is not a reflexive orthodisk system, there is an element $V$ of the tangent space $T_{\overrightarrow{\mathbf{t}}_{0}} \Delta$ for which $D_{V} \mathcal{H} \neq 0$.

This would then have the effect of proving that our critical point for the height function is reflexive, concluding the existence parts of the proofs of the main theorem.

We do not know how to prove or disprove this model statement in its full generality. On the other hand, it is not necessary for the proofs of the main theorems that we do so. Instead we will replace this theorem by a pair of lemmas. The lemmas each have two cases, with the division into cases depending on whether, in the configuration $\mathcal{C}=\mathrm{DH}_{m, n}$, we have $n=m$ or $m<n$. 
Lemma 5.1.2. Consider a configuration $\mathcal{C}=\mathrm{DH}_{m, n}$.

(i) Suppose $n=m$ and $\mathcal{Y} \subset \Delta$ is a real two-dimensional subspace of $\Delta$ which is defined by the equations $\mathcal{H}\left(t_{i}\right)=0$ for all coordinates $t_{i}$ with only two exceptions (say $t_{1}$ and $\left.t_{2}\right)$. Suppose also that the coordinates $t_{1}$ and $t_{2}$ refer to cycles which encircle neighboring edges, at most one of which is a finite edge. Then there is a sublocus $\mathcal{Y}^{*}$ along which $\mathcal{H}\left(t_{i_{1}}\right)=0$, and along that onedimensional sublocus, if $\overrightarrow{\mathbf{t}}_{0} \in \mathcal{Y}^{*}$ has positive height (i.e. $\mathcal{H}\left(\overrightarrow{\mathbf{t}}_{0}\right)>0$ ), there is an element $V \in T_{\overrightarrow{\mathbf{t}}_{0}} \mathcal{Y}^{*}$ of the tangent space $T_{\overrightarrow{\mathbf{t}}_{0}} \mathcal{Y}^{*}$ for which $D_{V} \mathcal{H} \neq 0$.

(ii) If $m<n$ and if $\mathcal{Y} \subset \Delta$ is a real one-dimensional subspace of $\Delta$ which is defined by the equations $\mathcal{H}\left(t_{i}\right)=0$ for all coordinates $t_{i}$ with only one exception (say $\left.t_{1}\right)$, and that exception refers to a cycle which is not a finite edge bully. Then if $\overrightarrow{\mathbf{t}}_{0} \in \mathcal{Y}$ has positive height, then there is an element $V \in T_{\overrightarrow{\mathbf{t}}_{0}} \mathcal{Y}$ of the tangent space $T_{\overrightarrow{\mathbf{t}}_{0}} \mathcal{Y}$ for which $D_{V} \mathcal{H} \neq 0$.

Lemma 5.1.3. Consider a configuration $\mathcal{C}=\mathrm{DH}_{m, n}$.

(i) If $n=m$, then for every configuration $\mathcal{C}=\mathrm{DH}_{m, n}$, there is a nonsingular analytic subspace $\mathcal{Y} \subset \Delta=\Delta_{\mathcal{C}}$, for which $\mathcal{Y}=\left\{\mathcal{H}\left(t_{i}\right)=0 \mid i \neq i_{1}, i_{2}\right\}$, for some choice of $i_{1}, i_{2}$, where $t_{i_{1}}$ and $t_{i_{2}}$ refer to cycles encircling neighboring edges, of which at most one is a finite edge.

(ii) If $m<n$, then for every configuration $\mathcal{C}=\mathrm{DH}_{m, n}$, there is a nonsingular analytic subspace $\mathcal{Y} \subset \Delta=\Delta_{\mathcal{C}}$, for which $\mathcal{Y}=\left\{\mathcal{H}\left(t_{i}\right)=0 \mid i \neq i_{1}\right\}$, for some choice of $i_{1}$ and the cycle referred to by $t_{i_{1}}$ is not a finite edge bully.

A cycle $\Gamma$ is a finite edge bully for the previous lemma if each finite edge $E$ of one of the orthodisks has a neighbor $E^{\prime}$ so that $\Gamma$ foots on $E$ but not $E^{\prime}$ or vice versa.

We introduce this concept because when $m+n$ is small, the technical requirements of the proof (see Lemma 5.3.1 and Definition 5.3.0) contrast with a shortage of sides; when $m+n \geq 5$ in case (ii), no cycle is a finite edge bully, and these hypotheses are vacuous. In our proof of Lemma 5.1.3, we will produce a cycle that is not a finite edge bully.

Given these lemmas, the proofs of Theorems A and B(i) are straightforward.

Proofs of Theorem A and Theorem B(i). Consider the locus $\mathcal{Y}$ (or the sublocus $\mathcal{Y}^{*}$ ) guaranteed by Lemma 5.1.3. By Theorem 4.5, the height function $\mathcal{H}$ is proper on $\mathcal{Y}\left(\right.$ resp. $\left.\mathcal{Y}^{*}\right)$, and has a critical point on $\mathcal{Y}$. By Lemma 5.1.2, this critical point represents a point where $\mathcal{H}=0$, i.e., a reflexive orthodisk by Lemma 4.5.5.

The proof of Lemma 5.1.2 occupies the current section while the proof of Lemma 5.1.3 is given in the following section. 
5.1.2. Let us discuss informally the proof of Lemma 5.1.2. Because angles of corresponding vertices in the $\Omega_{G d h} \leftrightarrow \Omega_{G^{-1}}$ dh correspondence sum to $0 \bmod 2 \pi$, the orthodisks locally topologically fit together along corresponding edges, so that conjugacy of orthodisks requires corresponding edges to move in different directions: if the edge $E$ on $\Omega_{G d h}$ moves "out", the corresponding edge $E^{*}$ on $\Omega_{G^{-1} d h}$ moves "in", and vice versa (see the figures below). Thus we expect that if $\gamma$ has an endpoint on $E$, then one of the extremal lengths of $\gamma$ decreases, while the other extremal length of $\gamma$ on the other orthodisk would increase: this will force the change of the height $\mathcal{H}(\gamma)$ of $\gamma$ to have a definite sign, as desired. This is the intuition behind Lemma 5.2.1; a rigorous argument requires us to actually compute derivatives of relevant extremal lengths using the formula (2.2) and we will need to avoid some technical difficulties involving singularities of the holomorphic quadratic differentials arising in (2.2). (We also will need to do some preliminary asymptotic analysis and an intermediate value theorem argument to find the sublocus $\mathcal{Y}^{*}$ along which to apply this argument in case (i).) We do this by displaying, fairly explicitly, the deformations of the orthodisks (in local coordinates on $\Omega_{G d h} / \Omega_{G^{-1} d h}$ ) as well as the differentials of extremal lengths, also in coordinates. After some preliminary notational description in $\S 5.2$, we do most of the computing in $\S 5.3$. Also in $§ 5.3$ is the key technical lemma, which relates the formalism of formula (2.2), together with the local coordinate descriptions of its terms, to the intuition we just described.

5.2. Deformations of $\mathrm{DH}_{1,1}$. We will prove Lemma 5.1.2 by first considering the special case of $\mathcal{C}=\mathrm{DH}_{1,1}$. We will then note that this case, defined in terms of precisely two curve systems and involving all the types of geometric situations encountered in all of the configurations $\mathcal{C}$, is only slightly different from the most general case: the arguments we give here extend immediately to prove the lemma in general, even though the exposition, in this specific low-dimensional case, can be much more concrete.

Here, we will describe the notation and results for this special case where $\mathcal{C}=\mathrm{DH}_{1,1}$.

We begin by recalling the notation of $\S 4.2$ : there the geometric coordinates were labeled $\Delta=(y, b, g)$ corresponding to the curve systems labeled yellow, blue, and green (see the figures in $\S 4.2$ ). We introduced a fourth curve system, mauve, which we used with blue to define the height function.

THEOREM 5.2.1. There exists a reflexive orthodisk system for the configuration $\mathrm{DH}_{1,1}$.

Proof. In this case, the statement of Lemma 5.1.3(i) is vacuous as the moduli space $\Delta=\Delta_{1,1}$ is already two-dimensional. So we are left to prove Lemma 5.1.2 in this case. The plan is simple, as we consider a path $\Gamma \subset \Delta$ 
which has two limits: towards one end $E$, the size of the period $H_{1} H_{2}-C_{1} P_{1}$ (and $H_{3} H_{2}-P_{3} C_{2}$ ) decays to zero, and towards the other end $E^{\prime}$, the period $P_{1} P_{2}-H_{1} C_{1}$ (and $P_{2} P_{3}-C_{2} H_{3}$ ) decays to zero. We

Claim 5.2.2.

$\operatorname{sgn}\left(\operatorname{Ext}_{\Omega_{G d h}}(\right.$ blue $)-\operatorname{Ext}_{\Omega_{G^{-1}} d h}($ blue $\left.)\right) \neq \operatorname{sgn}\left(\operatorname{Ext}_{\Omega_{G d h}}(\right.$ blue $)-\operatorname{Ext}_{\Omega_{G^{-1}} d h}($ blue $\left.)\right)$.

Assuming the claim, we continue with the proof of Theorem 5.2.1. As the sign of $\operatorname{Ext}_{\Omega_{G d h}}($ blue $)-\operatorname{Ext}_{\Omega_{G^{-1}} \text { dh }}$ (blue)) changes along the $\operatorname{arc} \Gamma=\{\Gamma(s)\}$, we know that there is a point $\Gamma\left(s_{0}\right)$ at which $\operatorname{Ext}_{\Omega_{G d h}}($ blue $)-\operatorname{Ext}_{\Omega_{G^{-1}} h}$ (blue) $)$ changes sign. Of course, this function $\operatorname{Ext}_{\Omega_{G d h}}$ (blue) $-\operatorname{Ext}_{\Omega_{G^{-1}} d h}$ (blue)) is the difference of real analytic regular functions of the coordinates on $\Delta$ (see expanded discussion of the analyticity of the curve at the end of the proof of Lemma 6.2), and so there is an analytic path through $\Gamma\left(s_{0}\right)$ along which $\operatorname{Ext}_{\Omega_{G d h}}$ (blue) $=\operatorname{Ext}_{\Omega_{G^{-1}} \text { dh }}$ (blue)). In keeping with the notations of Lemma 5.1.2 and 5.1.3, we denote that analytic path by $\mathcal{Y}^{*}$.

Our next task is to find a point on $\mathcal{Y}^{*}$ for which $\mathcal{H}$ (mauve) $=0$ : at such a point, we would have $\mathcal{H}$ (mauve) $=0=\mathcal{H}$ (blue). As $\mathcal{Y}^{*}$ is one-dimensional, and mauve is not a finite-edge bully, we in effect prove Lemma 5.1.2(i). The idea is to make an "infinitesimal push" along the edges $H_{1} H_{2}$ (and $H_{2} H_{3}$ ); we push $H_{1} H_{2}$ (and $H_{2} H_{3}$ ) "into" $\Omega_{G d h}$ and then the requirement that $\Omega_{G d h}$ and $\Omega_{G^{-1} d h}$ should remain conjugate then forces us to make an equal push of $H_{1} H_{2}$ (and $H_{2} H_{3}$ ) "out of" $\Omega_{G^{-1} d h}$. By the principle of domain monotonicity of extremal length, we expect the change in $\Omega_{G d h}$ to increase the extremal length $\operatorname{Ext}_{\Omega_{G d h}}$ (mauve) and we expect the change in $\Omega_{G^{-1} d h}$ to decrease the extremal length $\operatorname{Ext}_{\Omega_{G^{-1}} h}$ (mauve). The rigorously defined formulae and the technical Lemma 5.3.1 will support this intuition (indeed, will show that it is true to first order), and we find that if $\mathcal{H}$ (mauve) $\neq 0$ at a point $\overrightarrow{\mathbf{t}}_{0} \in \mathcal{Y}^{*}$, then there is an element $V \in T_{\overrightarrow{\mathbf{t}}_{0}} \mathcal{Y}^{*}$ of the tangent space $T_{\overrightarrow{\mathbf{t}}_{0}} \mathcal{Y}^{*}$ for which $D_{V} \mathcal{H} \neq 0$. This completes the proof of Lemma 5.1.2 in this case, as well as Theorem 5.1.2, up to the proof of the claim.

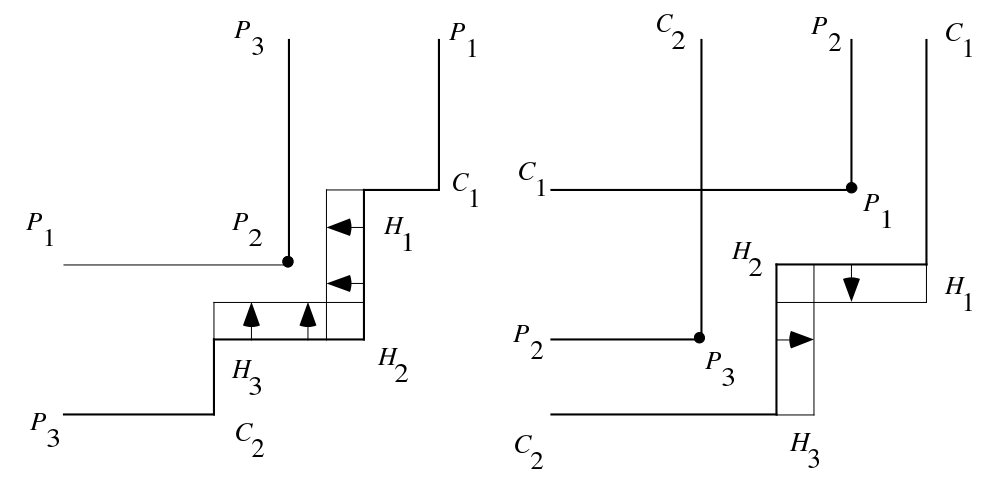

Pushing edges along the locus $\mathcal{Y}^{*}$ 
Proof of the Claim 5.2.2. It is evident that as the period $P_{1} P_{2}-H_{1} C_{1}$ tends to zero (with the period $H_{1} H_{2}-C_{1} P_{1}$ remaining bounded from below), the extremal length $\operatorname{Ext}_{\Omega_{G d h}}$ (blue) tends to a nonzero finite number, while the

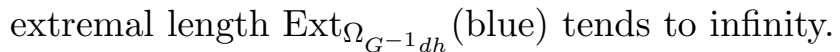

On the other hand, if we first send the period $P_{2} P_{3}-H_{1} H_{2}$ to zero and then allow the period $H_{1} H_{2}-P_{1} C_{1}$ to tend to zero, we see that $\operatorname{Ext}_{\Omega_{G d h}}$ (blue) is equal to the extremal length of blue on the quadrilateral component $P_{1}-P_{2}=$ $H_{2}-H_{1}-C_{1}-P_{1}$ of a noded surface, while $\operatorname{Ext}_{\Omega_{G^{-1}} \text { dh }}$ (blue) is given in terms of the extremal length of blue on the pentagonal surface $P-2-H_{2}-H_{1}-C_{1}-$ $P_{1}-P_{2}$. Yet the asymptotics of these extremal lengths of these explicit shapes are readily (if tediously) computable, and we find that $\operatorname{Ext}_{\Omega_{G d h}}$ (blue) decays to zero at a slower rate than $\operatorname{Ext}_{\Omega_{G^{-1}} \text { (bh }}$ (blue) as we send the period $H_{1} H_{2}-P_{1} C_{1}$ to zero. Thus by allowing $\Gamma$ to have ends near these two degenerate loci, we obtain the claim.

5.3. Infinitesimal pushes. We need to formalize the previous discussion. As always we are concerned with relating the Euclidean geometry of the orthodisks (which corresponds directly with the periods of the Weierstrass data) to the conformal data of the domains $\Omega_{G d h}$ and $\Omega_{G^{-1}} d h$. From the discussion above, it is clear that the allowable infinitesimal motions in $\Delta$, which is parametrized in terms of the Euclidean geometry of $\Omega_{G d h}$ and $\Omega_{G^{-1} d h}$, are given by infinitesimal changes in lengths of finite sides or in distances between sheets, with the changes being done simultaneously on $\Omega_{G d h}$ and $\Omega_{G^{-1} d h}$ to preserve conjugacy. The link to the conformal geometry is the formula (2.2): a motion which infinitesimally transforms $\Omega_{G d h}$, say, will produce an infinitesimal change in the conformal structure. Tensorially, this tangent vector to the moduli space of conformal structures is represented by a Beltrami differential. Later, formula (2.2) will be used, together with knowledge of the cotangent vectors $d \operatorname{Ext}_{\Omega_{G d h}}(\cdot)$ and $d \operatorname{Ext}_{\Omega_{G^{-1}} d h}(\cdot)$, to determine the derivatives of the relevant extremal lengths, hence the derivative of the height.

5.3.1. Infinitesimal pushes. Here we explicitly compute the effect of infinitesimal pushes of certain edges on the extremal lengths of relevant cycles. This is done by explicitly displaying the infinitesimal deformation and then using this formula to compute the sign of the derivative of the extremal lengths, using formula (2.2). There will be four different cases to consider, all of which are apparent in the $\mathrm{DH}_{1,1}$ orthodisks. This is why we can, without loss of generality, confine the computations to this case. To be concrete, we will choose for each case either the orthodisk $\Omega_{G d h}$ or the orthodisk $\Omega_{G^{-1} d h}$.

The three sides $H_{1} C_{1}, C_{1} P_{1}$ and $H_{2} H_{1}$ are geometrically different and require separate treatments. Thus we break our discussion up into cases. 
Case A. Finite noncentral edges (see also [WW]). Example: $H_{1} C_{1}$ in $\Omega_{G d h}$.

Case B. Infinite edge whose finite vertex is unbranched. Example: $H_{1} C_{1}$ in $\Omega_{G^{-1} d h}$.

Case C. Infinite edge whose finite vertex is a branch point off the symmetry line. Example: $C_{1} P_{1}$ on $\Omega_{G^{-1} d h}$.

Case D. An edge (finite or infinite) and its symmetric side meet in a corner. Example: $\mathrm{H}_{3} \mathrm{H}_{2}$ and $\mathrm{H}_{2} \mathrm{H}_{1}$.

For each case there are two subcases, which we can describe as depending on whether the given sides are horizontal or vertical. The distinction is, surprisingly, a bit important, as together with the fact that we do our deformations in pairs, it provides for an important cancellation of (possibly) singular terms affecting the formulation of Lemma 5.3.1. We defer this point for later (see Remark 5.3.3), while here we begin to calculate the relevant Beltrami differentials in the cases.

Also, each infinitesimal motion might require two different types of cases, depending on whether the edge we are deforming on $\Omega_{G d h}$ corresponds on $\Omega_{G^{-1} d h}$ to an edge of the same type or a different type. We will thus compute the Beltrami differentials only for a single domain, either $\Omega_{G d h}$ or $\Omega_{G^{-1} d h}$.

Case A. Here the computations are analogous to those that we found in [WW]; they differ only in orientation of the boundary of the orthodisk.
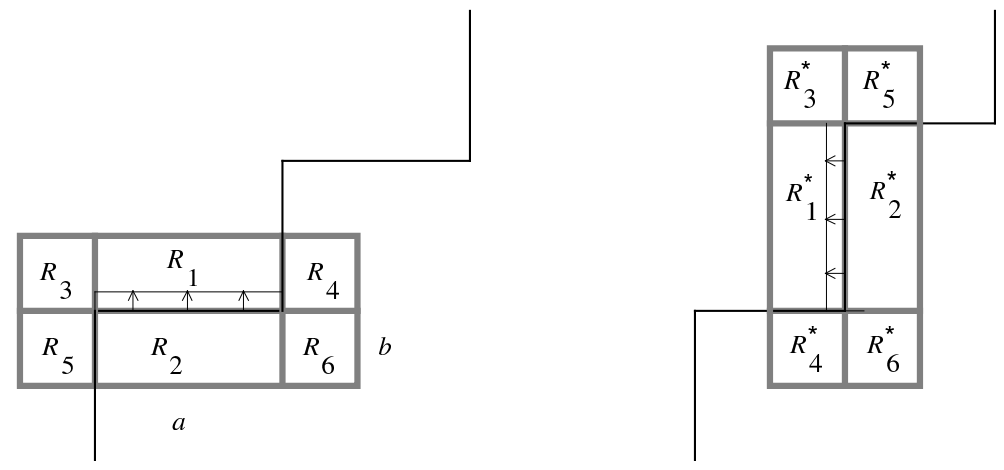

\section{Beltrami differential computation — Case A}

We first consider the case of a horizontal finite side; as in the figure above, we see that the neighborhood of the horizontal side of the orthodisk in the plane naturally divides into six regions which we label $R_{1}, \ldots, R_{6}$. Our deformation $f_{\varepsilon}=f_{\varepsilon, b, \delta}$ differs from the identity only in such a neighborhood, and in each of the six regions, the map is affine. In fact we have a two-parameter family of these deformations, all of which have the same infinitesimal effect, 
with the parameters $b$ and $\delta$ depending on the dimensions of the supporting neighborhood,

$$
f_{\varepsilon}(x, y)= \begin{cases}\left(x, \varepsilon+\frac{b-\varepsilon}{b} y\right), & \{-a \leq x \leq a, 0 \leq y \leq b\}=R_{1} \\ \left(x, \varepsilon+\frac{b+\varepsilon}{b} y\right), & \{-a \leq x \leq a,-b \leq y \leq 0\}=R_{2} \\ \left(x, y+\frac{\varepsilon+\frac{b-\varepsilon}{b} y-y}{\delta}(x+\delta+a)\right), & \{-a-\delta \leq x \leq-a, 0 \leq y \leq b\}=R_{3} \\ \left(x, y-\frac{\varepsilon+\frac{b-\varepsilon}{b} y-y}{\delta}(x-\delta-a)\right), & \{a \leq x \leq a+\delta, 0 \leq y \leq b\}=R_{4} \\ \left(x, y+\frac{\varepsilon+\frac{b+\varepsilon}{b} y-y}{\delta}(x+\delta+a)\right), & \{-a-\delta \leq x \leq-a,-b \leq y \leq 0\}=R_{5} \\ \left(x, y-\frac{\varepsilon+\frac{b+\varepsilon}{b} y-y}{\delta}(x-\delta-a)\right), & \{a \leq x \leq a+\delta,-b \leq y \leq 0\}=R_{6} \\ (x, y) & \text { otherwise }\end{cases}
$$

where we have defined the regions $R_{1}, \ldots, R_{6}$ within the definition of $f_{\varepsilon}$. Also note that here the orthodisk contains the arc $\{(-a, y) \mid 0 \leq y \leq b\} \cup\{(x, 0) \mid$ $-a \leq x \leq a\} \cup\{(a, y) \mid-b \leq y \leq 0\}$. Let $E$ denote the edge being pushed, defined above as $[-a, a] \times\{0\}$.

Of course $f_{\varepsilon}$ differs from the identity only on a neighborhood of the edge $E$, so that $f_{\varepsilon}$ takes the symmetric orthodisk to an asymmetric orthodisk. We next modify $f_{\varepsilon}$ in a neighborhood of the reflected (across the $y=-x$ line) segment $E^{*}$ in an analogous way with a map $f_{\varepsilon}^{*}$ so that $f_{\varepsilon}^{*} \circ f_{\varepsilon}$ will preserve the symmetry of the orthodisk.

Our present conventions are that the edge $E$ is horizontal; this forces $E^{*}$ to be vertical and we now write $f_{\varepsilon}^{*}$ for such a vertical segment; this is a straightforward extension of the description of $f_{\varepsilon}$ for a horizontal side, but we present the definition of $f_{\varepsilon}^{*}$ anyway, as we are crucially interested in the signs of the terms. So set

$$
f_{\varepsilon}^{*}= \begin{cases}\left(-\varepsilon+\frac{b-\varepsilon}{b} x, y\right), & \{-b \leq x \leq 0,-a \leq y \leq a\}=R_{1}^{*} \\ \left(-\varepsilon+\frac{b+\varepsilon}{b} x, y\right), & \{0 \leq x \leq b,-a \leq y \leq a\}=R_{2}^{*} \\ \left(x-\frac{-\varepsilon+\frac{b-\varepsilon}{\delta} x-x}{\delta}(y-\delta-a), y\right), & \{-b \leq x \leq 0, a \leq y \leq a+\delta\}=R_{3}^{*} \\ \left(x+\frac{-\varepsilon+\frac{b-\varepsilon}{b} x-x}{\delta}(y+\delta+a), y\right), & \{-b \leq x \leq 0,-a-\delta \leq y \leq-a\}=R_{4}^{*} \\ \left(x-\frac{-\varepsilon+\frac{b+\varepsilon}{b} x-x}{\delta}(y-\delta-a), y\right), & \{0 \leq x \leq b, a \leq y \leq a+\delta\}=R_{5}^{*} \\ \left(x+\frac{-\varepsilon+\frac{b+\varepsilon}{b} x-x}{\delta}(y+\delta+a), y\right), & \{0 \leq x \leq b,-a-\delta \leq y \leq-a\}=R_{6}^{*} \\ (x, y) & \text { otherwise. }\end{cases}
$$

Note that under the reflection across the line $\{y=-x\}$, the region $R_{i}$ gets taken to the region $R_{i}^{*}$. 
Let $\nu_{\varepsilon}=\frac{\left(f_{\varepsilon}\right)_{\bar{z}}}{\left(f_{\varepsilon}\right)_{z}}$ denote the Beltrami differential of $f_{\varepsilon}$, and set $\dot{\nu}=\left.\frac{d}{d \varepsilon}\right|_{\varepsilon=0} \nu_{\varepsilon}$. Similarly, let $\nu_{\varepsilon}^{*}$ denote the Beltrami differential of $f_{\varepsilon}^{*}$, and set $\dot{\nu}^{*}=\left.\frac{d}{d \varepsilon}\right|_{\varepsilon=0} \nu_{\varepsilon}^{*}$. Let $\dot{\mu}=\dot{\nu}+\dot{\nu}^{*}$. Now $\dot{\mu}$ is a Beltrami differential supported in a bounded domain in one of the domains $\Omega_{G d h}$ or $\Omega_{G^{-1} d h}$. We begin by observing that it is easy to compute that $\dot{\nu}=\left[\left.\frac{d}{d \varepsilon}\right|_{\varepsilon=0}\left(f_{\varepsilon}\right)\right]_{\bar{z}}$ evaluates near $E$ to

$$
\dot{\nu}= \begin{cases}\frac{1}{2 b}, & z \in R_{1} \\ -\frac{1}{2 b}, & z \in R_{2} \\ \frac{1}{2 b}[x+\delta+a] / \delta+i(1-y / b) \frac{1}{2 \delta}=\frac{1}{2 b \delta}(\bar{z}+\delta+a+i b), & z \in R_{3} \\ -\frac{1}{2 b}[x-\delta-a] / \delta-i(1-y / b) \frac{1}{2 \delta}=\frac{1}{2 b \delta}(-\bar{z}+\delta+a-i b), & z \in R_{4} \\ -\frac{1}{2 b}[x+\delta+a] / \delta+i(1+y / b) \frac{1}{2 \delta}=\frac{1}{2 b \delta}(-\bar{z}-\delta-a+i b), & z \in R_{5} \\ \frac{1}{2 b}[x-\delta-a] / \delta-i(1+y / b) \frac{1}{2 \delta}=\frac{1}{2 b \delta}(\bar{z}-\delta-a-i b), & z \in R_{6} \\ 0 & z \notin \operatorname{supp}\left(f_{\varepsilon}-\mathrm{id}\right) .\end{cases}
$$

We further compute

$$
\dot{\nu}^{*}= \begin{cases}-\frac{1}{2 b}, & R_{1}^{*} \\ \frac{1}{2 b}, & R_{2}^{*} \\ \frac{1}{2 b \delta}(i \bar{z}-\delta-a+b i) & R_{3}^{*} \\ \frac{1}{2 b \delta}(-i \bar{z}-\delta-a-b i) & R_{4}^{*} \\ \frac{1}{2 b \delta}(-i \bar{z}+\delta+a+b i) & R_{5}^{*} \\ \frac{1}{2 b \delta}(i \bar{z}+\delta+a-b i) & R_{6}^{*}\end{cases}
$$

Cases B, C. For both infinite sides the deformations are the same, here represented in the horizontal case. (We defer the case of infinite sides that meet along the symmetry line $\{y=-x\}$ until we treat Case D, where it will fit more naturally.)
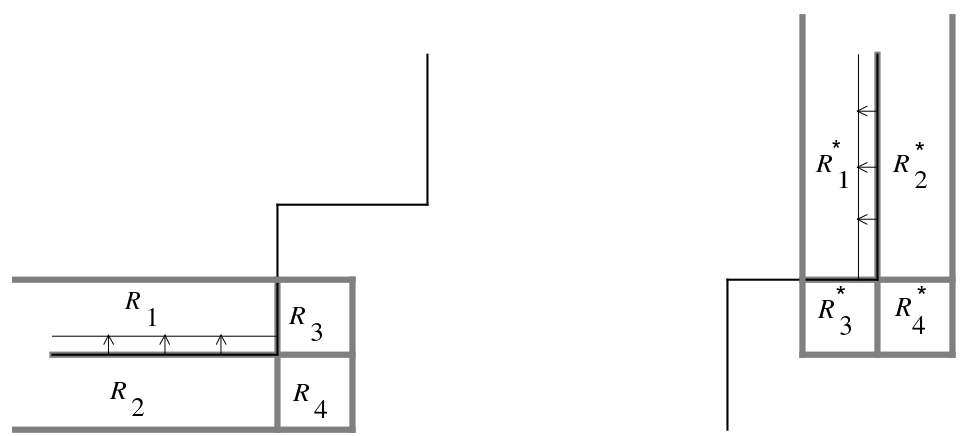

Beltrami differential computation - Cases B and C 


$$
f_{\varepsilon ; b, \delta}(x, y)= \begin{cases}\left(x, \varepsilon+\frac{b-\varepsilon}{b} y\right), & R_{1}=\{x \leq 0,0 \leq y \leq b\} \\ \left(x, \varepsilon+\frac{b+\varepsilon}{b} y\right), & R_{2}=\{x \leq 0,-b \leq y \leq 0\} \\ \left(x, y-\frac{\varepsilon+\frac{b-\varepsilon}{b} y-y}{\delta}(x-\delta)\right), & R_{3}=\{0 \leq x \leq \delta, 0 \leq y \leq b\} \\ \left(x, y-\frac{\varepsilon+\frac{b+\varepsilon}{b} y-y}{\delta}(x-\delta)\right), & R_{4}=\{0 \leq x \leq \delta,-b \leq y \leq 0\} \\ (x, y) & \text { otherwise. }\end{cases}
$$

Thus our infinitesimal Beltrami differential is given by

$$
\dot{\nu}_{b, \delta}= \begin{cases}\frac{1}{2 b}, & z \in R_{1} \\ -\frac{1}{2 b}, & z \in R_{2} \\ \frac{1}{2 b \delta}(-\bar{z}+\delta-i b), & z \in R_{3} \\ \frac{1}{2 b \delta}(\bar{z}-\delta-i b), & z \in R_{4} \\ 0 & z \notin \operatorname{supp}\left(F_{\varepsilon ; b, \delta}-\mathrm{id}\right) .\end{cases}
$$

The formulas for a push of a vertical edge are analogous.

Case D. We have separated this case out for purely expositional reasons. We can imagine that the infinitesimal push that moves the pair of consecutive sides along the symmetry line $\{y=-x\}$ is the result of a composition of a pair of pushes from Case A or from Case C; i.e., our diffeomorphism $F_{\varepsilon ; b, \delta}$ can be written $F_{\varepsilon ; b, \delta}=f_{\varepsilon} \circ f_{\varepsilon}^{*}$, where the maps differ from the identity in the union of the supports of $\dot{\nu}_{b, \delta}$ and $\dot{\nu}_{b, \delta}^{*}$.

It is an easy consequence of the chain rule applied to this formula for $F_{\varepsilon ; b, \delta}$ that the infinitesimal Beltrami differential for this deformation is the $\operatorname{sum} \dot{\nu}_{b, \delta}+\dot{\nu}_{b, \delta}^{*}$ of the infinitesimal Beltrami differentials $\dot{\nu}_{b, \delta}$ and $\dot{\nu}_{b, \delta}^{*}$ defined in formula (5.2) for Case A (even in a neighborhood of the vertex along the diagonal where the supports of the differentials $\dot{\nu}_{b, \delta}$ and $\dot{\nu}_{b, \delta}^{*}$ coincide).

5.3.2. Derivatives of extremal lengths. In this section, we combine the computations of $\dot{\nu}_{b, \delta}$ with formula (2.2) (and its background in $\S 2$ ) and some easy observations on the nature of the quadratic differentials $\Phi_{\mu}=\frac{1}{2} d \operatorname{Ext}_{(\cdot)}(\mu) \mid$. to compute the derivatives of extremal lengths under our infinitesimal deformations of edge lengths.

We begin by recalling some background from $\S 2$. If we are given a curve $\gamma$, the extremal length of that curve on an orthodisk, say $\Omega_{G d h}$, is a real-valued $C^{1}$ function on the moduli space of that orthodisk. Its differential is then a holomorphic quadratic differential $\Phi_{\gamma}=\left.\frac{1}{2} d \operatorname{Ext}_{(\cdot)}(\gamma)\right|_{\Omega_{G d h}}$ on that orthodisk; the horizontal foliation of $\Phi_{\gamma}$ consists of curves which connect the same edges in $\Omega_{G d h}$ as $\gamma$, since $\Phi_{\gamma}$ is obtained as the pullback of the quadratic differential 
$d z^{2}$ from a rectangle where the image of $\gamma$ connects the opposite vertical sides. We compute the derivative of the extremal length function using formula (2.2); i.e.

$$
\left(\left.d \operatorname{Ext}(\gamma)\right|_{\Omega_{G d h}}\right)[\nu]=4 \operatorname{Re} \int_{\Omega_{G d h}} \Phi_{\gamma} \nu
$$

It is here where we find that we can actually compute the sign of the derivative of the extremal lengths, hence the height function, but also encounter a subtle technical problem. The point is that we will discover that, in suitable circumstances, just the topology of the curve $\gamma$ on $\Omega_{G d h}$ will determine the sign of the derivative on an edge $E$, so we will be able to evaluate the sign of the integral above, if we shrink the support of the Beltrami differential $\dot{\nu}_{b, \delta}$ to the edge by sending $b, \delta$ to zero. (In particular, the sign of $\Phi_{\gamma}$ depends precisely on whether the foliation of $\Phi=\Phi_{\gamma}$ is parallel or perpendicular to $E$, and on whether $E$ is horizontal or vertical.) We then need to know two things: 1) that this limit exists, and 2) that we may know its sign via examination of the sign of $\dot{\nu}_{b, \delta}$ and $\Phi_{\gamma}$ on the edge $E$. We phrase this as follows:

Definition 5.3.0. $\Phi$ is admissable on an edge $E$ if and only if at a vertex $v$ of $E$ and in a conformal parameter $z$ centered at $v$, we have $|z| \Phi(z)=o(1)$.

Lemma 5.3.1. Let $\Phi$ be admissable for the edge $E$. (1) $\lim _{b \rightarrow 0, \delta \rightarrow 0} \operatorname{Re} \int \Phi \dot{\nu}$ exists, is finite and nonzero. (2) The foliation of $\Phi$ is either parallel or orthogonal to the interior of the segment which is $\lim _{b \rightarrow 0, \delta \rightarrow 0}(\operatorname{supp} \dot{\nu})$, and (3) The expression $\Psi \dot{\nu}$ has a constant sign on that segment $E$, and the integral (2.2) also has that (same) sign.

In the statement of the lemma, the foliation refers to the horizontal foliation of the holomorphic quadratic differential $\Phi=\Phi_{\gamma}$, whose core curve is $\gamma$.

This lemma provides the rigorous foundation for the intuition described in the paragraph of strategy $\S 5.1 .2$.

\subsubsection{Proof of the technical lemma and a remark on its limitations.}

Proof of Lemma 5.3.1. The foliation of $\Phi$, on say $\Omega_{G d h}$, lifts to a foliation on the punctured sphere (which we will denote by $S_{G d h}$ ), symmetric about the reflection about the equator. This proves the second statement. The third statement follows from the first (and from the above discussion of the topology of the vertical foliation of $\Phi_{\gamma}$, once we prove that there is no infinitude coming from either the neighborhood of infinity of the infinite edges or the regions $R_{3}$ and $R_{4}$ for the finite vertices. This finiteness will follow from the proof of the first statement. Thus, we are left to prove the first statement. 
In the case of an edge with a finite vertex, we estimate the contribution to $\operatorname{Re} \int \Phi \dot{\nu}$ of a neighborhood $N$ of a vertex from regions $R_{1}$ and $R_{2}$ (and $R_{1}^{*}$ and $R_{2}^{*}$ ) as

$$
\begin{aligned}
\left|\operatorname{Re} \int \Phi \dot{\nu}\right| & \leq \int_{0}^{b} \int_{N \cap \partial \Omega_{G d h}} \frac{1}{2 b} \cdot|\Phi| d x d y \\
& =\frac{1}{2 b} \int_{0}^{b} \int_{N \cap \partial \Omega_{G d h}} o\left(x^{2}+y^{2}\right)^{-1 / 2} d x d y
\end{aligned}
$$

which converges by the dominated convergence theorem. The other regions are analogous.

In the case of an edge with an infinite vertex, the argument is somewhat easier, as the possible growth of $|\Phi|$ as we tend towards the vertex has an a priori bound of rapid decay. We compute this decay by observing that in the coordinate $w$ of the plane, the quadratic differential $\Phi$ has expansion $\Phi=c_{1} / w+\{$ h.o.t. $\} d w$, and the Schwarz-Christoffel map from the half-plane to the orthodisk has integrand $\omega=\left\{c_{0} z^{-3 / 2}+\right.$ h.o.t. $\} d z$. It is then straightforward that in the coordinate $z$ of the orthodisk, the quadratic differential $\Phi$ decays faster than $|z|^{-2}$.

Computations like those in the final paragraph of the above proof prove the

Proposition 5.3.2. The holomorphic quadratic differential $\Phi$ is admissable on an edge $E$ if $\Phi$ relates to the vertices $v_{1}$ and $v_{2}$ of $E$ in one of the following ways:

(i) The orthodisk has an angle of $\pi / 2$ of $v_{i}$.

(ii) The vertex $v_{i}$ is at infinity.

(iii) The orthodisk has an angle of $3 \pi / 2$ at $v_{i}$, and the foliations of $\Phi$ are either both parallel to the edges incident to $v_{i}$ or both orthogonal for those edges.

(iv) The vertex $v_{i}$ is a branch point where the orthodisk makes an angle of $5 \pi / 2$, and the holomorphic quadratic differential has a singularity at $v_{i}$.

In the last case (iv), a singularity arises if and only if the foliation is parallel to the edges incident to $v_{i}$ and a leaf emanates from $v_{i}$, or the foliation is orthogonal to those edges and at least two leaves emanate from $v_{i}$.

Proof. As an example, we discuss (iii). Here, an appropriate branch of the map $z \mapsto z^{2 / 3}=w$ takes a neighborhood of $v_{i}$ in the orthodisk to a neighborhood of the origin in the upper half-plane. The condition on the foliation on the proposition allow us to conclude [Str] that the holomorphic quadratic differential $\Phi$ has the expansion in the upper half-plane as $\left(c_{0}+c_{1} z+\right.$ h.o.t. $) d z^{2}$, from which the statement follows. 
Remark 5.3.3. In light of the previous proposition, we see that it is quite common to have an edge which is not admissable for a holomorphic quadratic differential: for instance we need only consider the differential of extremal length for a cycle which has feet on an isolated edge with a vertex where the orthodisk has angle at least $3 \pi / 2$. Thus, it is a significant problem to find extremal lengths so that Lemma 5.3.1 may be invoked, and this restriction informed our choice of height function after Definition 4.5.2. In particular, we were careful not to have the special cycles referred to in Lemma 5.1.2 have feet adjacent to edges where we would later push.

Moreover, in the cases where an edge $E$ is not admissable for a given holomorphic quadratic differential, we cannot - in general - expect to have control over Re $\int_{E} \Phi \dot{\nu}$. This follows from an analysis similar to that in the proof of the previous proposition or in the final paragraph of the proof of Lemma 5.3.1: by choosing a branch of the map from a neighborhood of a vertex to the upper half-plane, it is easy to see that there is no guarantee that $\lim _{b, \delta \rightarrow 0} \operatorname{Re} \int \Phi \dot{\nu}_{b, \delta}$ exists. However, a choice of consistent branches of those maps from a neighborhood $N(v)$ of a vertex $v$ and from the reflection $N\left(v^{*}\right)$ of $N(v)$ across the central diagonal show that $\operatorname{Re}\left[\int_{N(v)} \Phi \dot{\nu}_{b, \delta}+\int_{N\left(v^{*}\right)} \Phi \dot{\nu}_{b, \delta}^{*}\right]=$ $O(1)$ as $b, \delta \rightarrow 0$; i.e., the contributions from the terms in the expansions of $\Phi$ of order $z^{-\alpha}$ from $\alpha \geq 1$ cancel in pairs. Thus, while we then find that $\lim _{b, \delta \rightarrow 0} \operatorname{Re} \int \Phi \dot{\nu}_{b, \delta}$ exists — which we expect from $\dot{\nu}_{b, \delta}$ always representing a unique tangent vector to $\Delta$ - we also find that the contributions of the terms $z^{-\alpha}(\alpha \geq 1)$ to the sign of $\Phi$ on nearby edges must be ignored in evaluating $\lim _{b, \delta \rightarrow 0} \operatorname{Re} \int \Phi \dot{\nu}_{b, \delta}$.

Conclusion of the proof of Theorem 5.2.1. Conjugacy of the domains $\Omega_{G d h}$ and and $\Omega_{G^{-1} d h}$ requires that if we push an edge $E \subset \partial \overline{\Omega_{G d h}}$ into the domain $\Omega_{G d h}$, we will change the Euclidean geometry of that domain in ways that will force us to push the corresponding edge $E^{*} \subset \partial \overline{\Omega_{G^{-1}} d h}$ out of the domain $\Omega_{G^{-1} d h}$. To see this, observe that for finite length edges, the fact that the angles add to multiples of $2 \pi$ (see the table in $§ 3.4$ ) means that there is a pair of homeomorphisms of $\Omega_{G d h}$ and $\Omega_{G^{-1} d h}$, respectively, which preserves the verticality or horizontality of the bounding edges, but allows the two-image domains $\Omega_{G d h}{ }^{*}$ and $\Omega_{G^{-1} d h}{ }^{*}$, respectively, to fit together to form a locally Euclidean space. (We can also require the homeomorphisms to preserve the lengths of finite boundary edges; the domains will continue to fit together as above.) We first discuss the situation of pushes of finite boundary edges. Conjugacy requires that $\Omega_{G d h}$ and $\Omega_{G^{-1}}$ dh have the same Euclidean lengths, and for finite edges, this length is but a single measurement in the Euclidean complex $\Omega_{G d h}{ }^{*} \cup \Omega_{G^{-1} d h}{ }^{*}$. But in this complex $\Omega_{G d h}{ }^{*} \cup \Omega_{G^{-1} d h}{ }^{*}$, a push out of a finite edge of $\Omega_{G d h}{ }^{*}$ is simultaneously a pushing in of the corresponding 
edge of $\Omega_{G^{-1} d h}{ }^{*}$, and vice versa. Thus, because our homeomorphisms could be taken to preserve a neighborhood of the boundary of the domains $\Omega_{G d h}$ and and $\Omega_{G^{-1} d h}$, we see that a push out of a finite edge of $\Omega_{G d h}$ is simultaneously a pushing in of the corresponding edge of $\Omega_{G^{-1} d h}$, and vice versa.

The corresponding statement for infinite edges is argued analogously, once we remark that in the above argument about finite edges, (i) we never made use of the lengths of the (finite) edges being pushed, so this quantity is irrelevant to the discussion, and (ii) we also never made formal use of the cone angles being at finite points in the plane - the arguments applied equally well to boundaries of $\Omega_{G d h}$ and $\Omega_{G^{-1} d h}$ fitting together at $\infty$.

We apply this general reasoning to the case of $\mathcal{C}=\mathrm{DH}_{1,1}$. Our only pushing is along the locus $\mathcal{Y}^{*}=\{\mathcal{H}($ blue $)=0\}$, and we seek to push $H_{1} H_{2}$ and $\mathrm{H}_{2} \mathrm{H}_{3}$. Here the measured foliations for the holomorphic quadratic differentials $\Phi_{\text {mauve }}=\frac{1}{2} d \operatorname{Ext}_{\Omega}$. (mauve) are parallel to the sides being pushed and to the neighboring sides. Proposition 5.3.2(i),(iii) then assert that the quadratic differentials are admissible for those edges; Lemma 5.3.1 then implies that since the Beltrami differentials $\dot{\nu}_{\Omega}$. have opposite signs on corresponding edges, so do $\Phi_{\text {mauve }} \dot{\nu}_{\Omega}$; thus

$$
\operatorname{sgn} d \operatorname{Ext}_{\Omega_{G d h}}(\text { mauve })\left[\dot{\nu}_{\Omega_{G d h}}\right]=-\operatorname{sgn} d \operatorname{Ext}_{\Omega_{G^{-1}} d h}(\text { mauve })\left[\dot{\nu}_{\Omega_{G^{-1}} d h}\right] .
$$

This concludes the proof of Lemma 5.1.2 in this case, and hence also the proof of Theorem 5.2.1.

Conclusion of the proof of Lemma 5.1.2. We begin with statement (i). The general case of this statement is precisely analogous to the case of Theorem 5.2.1; indeed, we can regard the locus $\mathcal{Y}$ from Lemma 5.1.2 as being a version of the moduli space $\Delta$ studied in Theorem 5.2.1. The coordinates $t_{i_{1}}$ and $t_{i_{2}}$ from the lemma are given by periods $H_{1} C_{1}-P_{1} P_{2}$ exactly as in the treatment of $\Delta_{1,1}$. In particular, the asymptotic estimates calculated in the proof of Claim 5.2.2 continue to hold exactly as before, as the setting for those estimates has changed in only insubstantial ways. (To see this, note that the only changes are the we do not necessarily have that $P_{2}$ tends to $H_{2}$ on $\Omega_{G d h}$ (which does not affect the estimates since we continue to have $\left|H_{1} C_{1}-P_{1} P_{2}\right|$ bounded away from zero), and the angle at $P_{2}$ on $\Omega_{G d h}$ has changed (also immaterially as $P_{2}$ is at some bounded distance from where the degeneracy is occurring).)

Moreover, because the rest of the terms of $\mathcal{H}_{\mathcal{C}}$ vanish along the locus $\mathcal{Y}$ to second order in the deformation variable, we see that any deformation of the orthodisk will not alter the contribution of these terms to $\mathcal{H}_{\mathcal{C}}$. Thus the only effect of an infinitesimal deformation of an orthodisk system on $\mathcal{Y}$ to the height function $\mathcal{H}_{\mathcal{C}}$ is to the terms $\mathcal{H}\left(t_{i_{1}}\right)$ and $\mathcal{H}\left(t_{i_{2}}\right)$, which we control as in Theorem 5.2.1. 
For statement(ii) of the lemma, we deform as we did along the locus $\mathcal{Y}^{*} \subset \mathcal{Y}$, here pushing on a finite edge which is parallel to the foliation for $\Phi_{t_{i}}$ (in the obvious notation) if and only its neighbors are. (It is worth remarking that in the case of $\mathrm{DH}_{1,2}$, we will not be able to find an edge which is finite on both $\Omega_{G d h}$ and $\Omega_{G^{-1} d h}$; in this case we will push on $H_{1} C_{1}$, which, relative to the cycle $H_{2} H_{3}-P_{1} P_{2}$ is admissible by Proposition 5.3.2(i),(ii). Thus the proof above for the case $m=n$ also extends to this case.)

This concludes the proof of the lemma.

\section{Regeneration}

In the previous section we showed how we might reduce the height function $\mathcal{H}_{\mathcal{C}}$ at a critical point of a smooth locus $\mathcal{Y}$, where the locus $\mathcal{Y}$ was defined as the null locus of all but two of the heights, say $\mathcal{H}\left(t_{i_{1}}\right)$ and $\mathcal{H}\left(t_{i_{2}}\right)$. In this section, we prove Lemma 5.1.3, which guarantees the existence of such a locus $\mathcal{Y}$.

We will continue with the rather concrete exposition we used in $\S 5$; i.e., we will prove the existence of the genus five Costa tower, $\mathrm{DH}_{2,2}$ by using Theorem 5.2.1 (the existence of the genus three Costa tower, $\mathrm{DH}_{1,1}$ ) to imply the existence of a locus $\mathcal{Y} \subset \Delta=\Delta_{2,2}$ - Lemma 5.1.2 and the properness Theorem 4.5.1 then prove the existence. The arguments we give will immediately generalize to prove the existence of a genus $2 n+1$ Costa tower $\mathrm{DH}_{n, n}$, given the existence of a genus $g=2 n-1$ Costa tower $\mathrm{DH}_{n-1, n-1}$.

Indeed, our proof of Theorem A is by induction:

Inductive Assumption A. There exists a genus $2 n-1$ Costa tower, $\mathrm{DH}_{n-1, n-1}$.

Thus, all of our surfaces are produced from only slightly less complicated surfaces; this is the general principle of 'handle addition' referred to in the title.

For concreteness, our present goal is the proof of the statement:

THEOREM 6.1. There is a reflexive orthodisk system for the configuration $\mathrm{DH}_{2,2}$.

Start of proof. Let us use the given height $\mathcal{H}_{2,2}$ for $\mathrm{DH}_{2,2}$ and consider how the height $\mathcal{H}_{1,1}$ for $\mathrm{DH}_{1,1}$ relates to it, near a solution $X_{1}$ for the $\mathrm{DH}_{1,1}$ problem. (As we observed, the situation extends to general $\mathrm{DH}_{n, n}$ and $\mathrm{DH}_{n-1, n-1}$ with simple added notation.)

Our notation is given in $\S 4.4$ and is recorded in the diagrams below: for instance, the curve system $\delta$ connects the edges $P_{1} P_{2}$ and $H_{2} H_{3}$.

We are very interested in how an orthodisk system might degenerate. One such degeneration is shown in the next figure, where the branch point 
$P_{1}$ has collided with the vertex $H_{1}$ on the orthodisk $\Omega_{G^{-1} d h}$. This has the effect, on the orthodisk $\Omega_{G d h}$ of causing $H_{1} C_{1}$ to collapse and forcing the line $P_{1} P_{2}$ (respectively, $P_{4} P_{5}$ ) to lie directly across from (resp., over) the point $H_{1}=C_{1}$ (resp., $C_{2}=H_{5}$ ). The point is that the degenerating family of (pairs of) Riemann surfaces in $\Delta_{\mathcal{C}}$ limits on (a pair of) surfaces with nodes. (We recall that a surface with nodes is a complex space where every point has a neighborhood complex isomorphic to either the disk $\{|z|<1\}$ or a pair of disks $\left\{(z, w) \in \mathbb{C}^{2} \mid z w=0\right\}$.) In the case of the surface corresponding to $\Omega_{G^{-1} d h}$, the components of the noded surface (i.e. the regular components of the noded surface in the complement of the nodes) are difficult to observe, as the flat structures on the thrice-punctured sphere components are simply single points; on the other hand, in the case of the surface corresponding to $\Omega_{G d h}$, the nodes are quite visible, as coming from a pair of sheets on the orthodisk, with one infinite point and one finite point (drawn in paler lines on the figure below on the right).
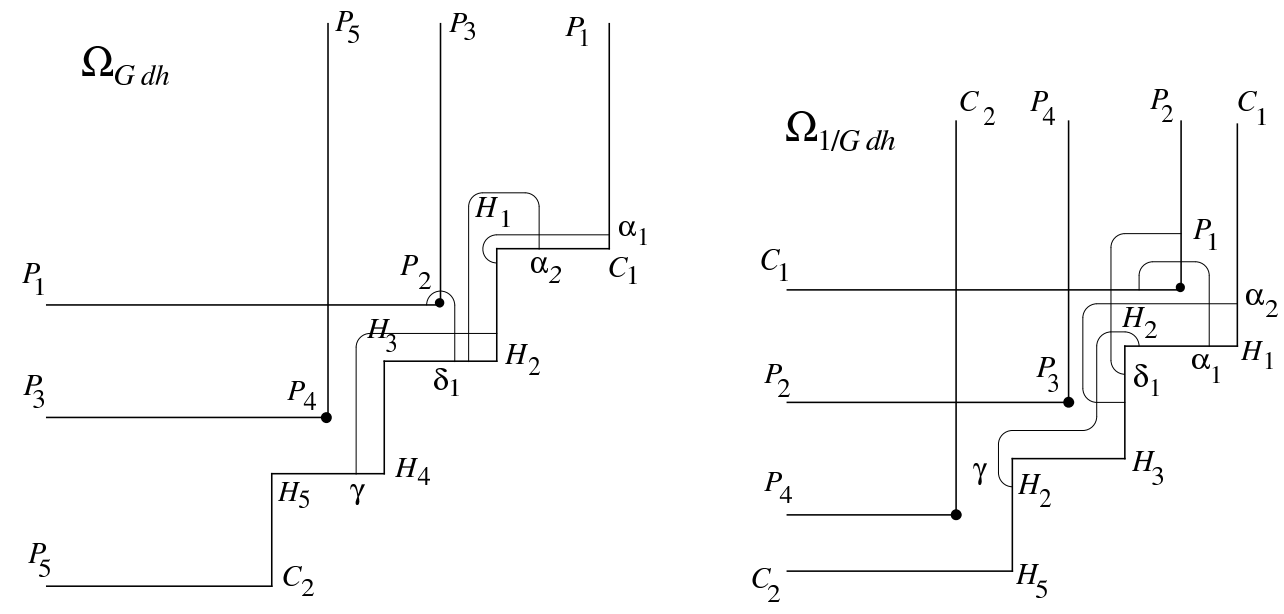

Curve system used for regeneration

Remark. In all of this, it is important to keep in mind that our flat structures represent a Riemann surface with a meromorphic one-form. Thus while there are no nontrivial holomorphic one-forms on a sphere (so that the flat structure is given by the trivial form $0 \cdot d z$, and is hence a point), there are nontrivial meromorphic forms on a thrice-punctured sphere with poles only at the punctures. The flat structures for those that occur in our present situation are represented in our method by a bigon, with one finite and one infinite vertex, connected by one horizontal and one vertical side. 
An important issue in this section is that some of our curves cross the pinching locus on the surface, i.e. the curve on the surface which is being collapsed to form the node. In particular, in the diagram, the curves $\mu$ and $\nu$ are such curves, and so their depiction in the degenerated figure is, well, degenerate; the curves either surround a point, or connect a point and an edge, or connect a pair of edges on opposite sides of a node.

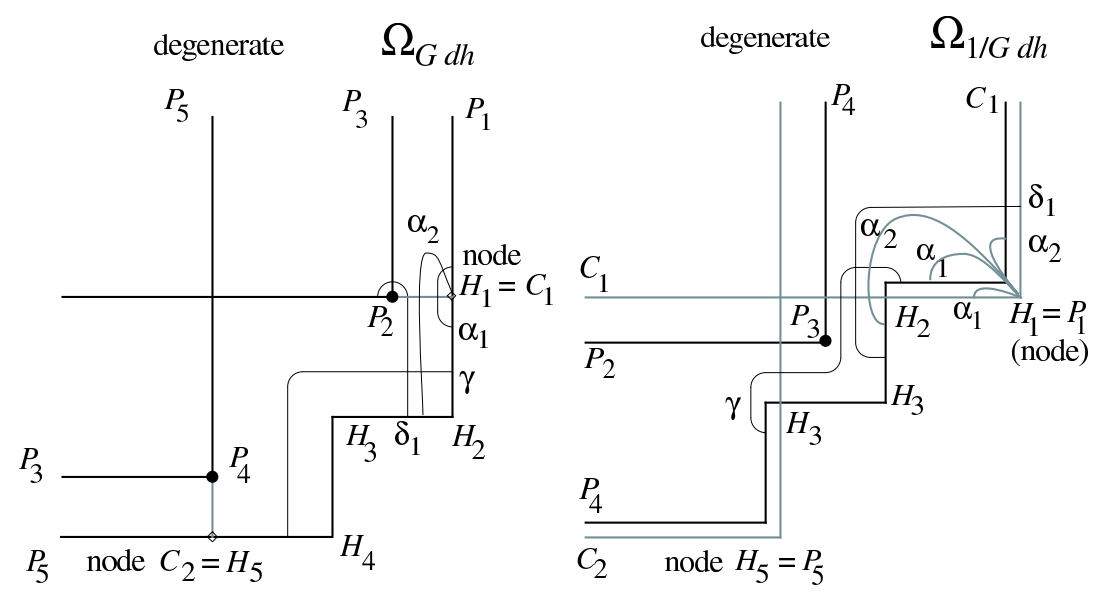

Degenerated orthodisks for the Costa towers

When we remove the thrice-punctured sphere components, we observe (compare with the figures in $\S 5.2$ and $\S 3.6$ ) that we are left with an orthodisk system for the lower genus configuration $\mathcal{C}_{\mathrm{DH}_{1,1}}$, up to possibly relabeling $\Omega_{G d h}$ as $\Omega_{G^{-1} d h}$ and vice versa.

Our basic approach is to work backward from this understanding of degeneration - we aim to "regenerate" the locus $\mathcal{Y}$ in $\Delta_{\mathrm{DH}_{2,2}}$ from the solution $X_{1} \in \Delta_{\mathrm{DH}_{1,1}}$.

We focus on the curves $\delta$ and $\gamma$, ignoring the degenerate curves $\mu$ and $\nu$. Let $\Delta_{2}$ be the geometric coordinate simplex for $\mathrm{DH}_{2,2}$.

(In the general case for $\Delta_{\mathrm{DH}_{n, n}}$, there are $2 n-2$ nondegenerate curves (say, for notational convenience $\left\{b_{1}, \ldots, b_{2 n-2}\right\}$ ), and two degenerate curves, $\mu$ and $\nu$. The case of $\mathrm{DH}_{m, n \neq m}$ requires a separate but parallel treatment.)

We restate Lemma 5.1.3 in terms of the present (simpler) notation.

Lemma 6.2 (Regeneration). There is a smooth two-dimensional analytic closed locus $\mathcal{Y} \subset \overline{\Delta_{n}}$ so that $\operatorname{Ext}_{\Omega_{G d h}}\left(b_{i}\right)=\operatorname{Ext}_{\Omega_{G^{-1}} \text { dh }}\left(b_{i}\right)$ on $\mathcal{Y}$, and $\mathcal{Y}$ is proper in $\Delta_{n}$.

Proof. We again continue with the notation for $n=2$, as the general situation follows with just added notation. 
As putatively defined in the statement of the lemma, the locus $\mathcal{Y}$ would be clearly closed, and would have nonempty intersection with $\overline{\Delta_{2}}$ as $\overline{\Delta_{2}}$ contains the solution $X_{1}$ to the genus 3 problem.

We parametrize $\overline{\Delta_{2}}$ near $X_{1}$ as $\Delta_{1} \times[(0, \varepsilon) \times(0, \varepsilon)]$ and consider the map

$$
\Phi:\left(X,\left(t_{1}, t_{2}\right)\right): \Delta_{1} \times[(0, \varepsilon) \times(0, \varepsilon)] \longrightarrow \mathbb{R}^{2}
$$

given by

$$
\left(X,\left(t_{1}, t_{2}\right)\right) \mapsto\left(\operatorname{Ext}_{\Omega_{G d h}}(\delta)-\operatorname{Ext}_{\Omega_{G^{-1}} d h}(\delta), \operatorname{Ext}_{\Omega_{G d h}}(\gamma)-\operatorname{Ext}_{\Omega_{G^{-1}} d h}(\gamma)\right) .
$$

Here, coordinates $t_{1}$ and $t_{2}$ refer to a specific choice of normalized geometric coordinate; i.e., $t_{1}=\operatorname{Im}\left(P_{5} C_{2} \longrightarrow H_{5} H_{4}\right)$ and $t_{2}=\operatorname{Re}\left(C_{2} H_{5} \longrightarrow P_{4} P_{5}\right)$, where the periods $P_{5} C_{2} \longrightarrow H_{5} H_{4}$ and $C_{2} H_{5} \longrightarrow P_{4} P_{5}$ are measured on the domain $\Omega_{G d h}$ (see the figure below). In terms of these coordinates, we note that whenever either $t_{1}=0$ or $t_{2}=0$, we are in a boundary stratum of $\overline{\Delta_{2}}$. The locus $\left\{t_{1}>0, t_{2}>0\right\} \subset \overline{\Delta_{2}}$ is a neighborhood in $\operatorname{Int}\left(\Delta_{2}\right)$ with $X_{1}$ in its closure.

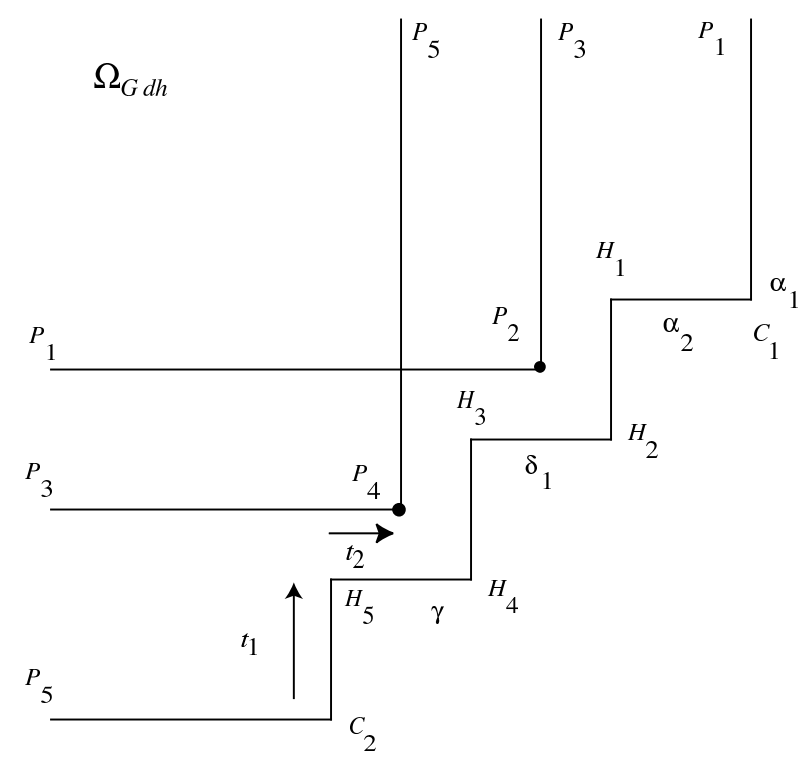

Geometric coordinates $t_{1}$ and $t_{2}$ for $\Delta_{2}$

Note that $\Phi\left(X_{1},(0,0)\right)=0$ as $X_{1}$ is reflexive.

Now, to find the locus $\mathcal{Y}$, we apply the implicit function theorem, which says that if

(i) the map $\Phi$ is differentiable, and

(ii) the differential $\left.d \Phi\right|_{T_{X_{1}} \Delta_{1}}$ is an isomorphism onto $\mathbb{R}^{2}$,

then there exists a differentiable family $\mathcal{Y} \subset \overline{\Delta_{2}}$ for which $\left.\Phi\right|_{\mathcal{Y}} \equiv 0$. 
In order to show the differentiability condition (i), we require a clarification as to the meaning of the curve system $\gamma$, for points in $\Delta_{1} \subset \partial \overline{\Delta_{2}}$. Here on $X_{1}$ we consider the distinguished points $x$ and $x^{\prime}$ on the $\Omega_{G^{-1} d h}$ orthodisk, as shown below.

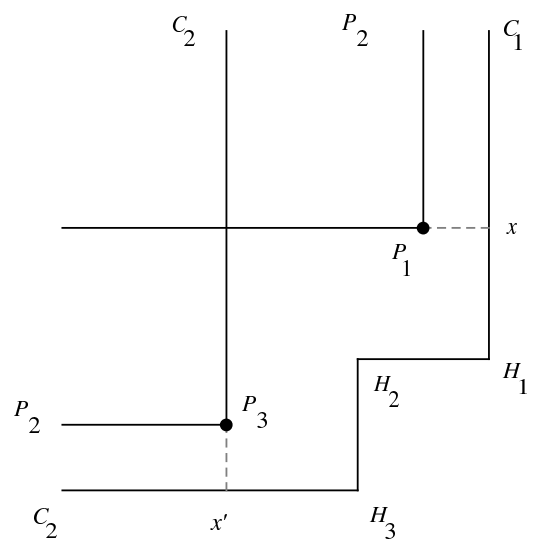

Definitions of the points $x$ and $x^{\prime}$

By definition, these points $x$ and $x^{\prime}$ on $\Omega_{G^{-1}} d h$ of the solution $X_{1}$ are determined from continuing the levels $C_{1} P_{1}$ and $P_{3} C_{2}$ until they meet the axis at $H_{1} C_{1}$ and $C_{2} H_{3}$, respectively. Then $\gamma$ is defined on $\Delta_{1} \subset \partial \overline{\Delta_{2}}$ as the curve family connecting $H_{1} x$ and $H_{3} x^{\prime}$. We extend the notion of the points $x$ and $x^{\prime}$ to the interior of $\Delta_{2}$ by declaring there that $x=H_{1}$ and $x^{\prime}=H_{5}$, respectively. The definition of $\gamma$ then extends continuously to $\overline{\Delta_{2}}$.

We now prove the differentiability (condition (i)) of $\Phi$. As the locus of $\overline{\Delta_{2}} \in \widehat{\overline{\mathcal{M}}}_{5} \times \widehat{\overline{\mathcal{M}}}_{5}$ is differentiable (here $\widehat{\overline{\mathcal{M}}}_{5}$ refers to a smooth cover of the relevant neighborhood of $X_{1} \subset \overline{\mathcal{M}}_{5}$, where $\overline{\mathcal{M}}_{5}$ is the Deligne-Mostow compactification of the moduli space of curves of genus five) the theorem of Gardiner-Masur [GM] implies that $\Phi$ is differentiable, as we have been very careful to choose curves $\{\delta, \gamma\}$ which are nondegenerate in a neighborhood of $\overline{\Delta_{2}}$ near the genus three solution $X_{1}$, with both staying in a single regular component of the noded surface.

We are left to treat (ii), the invertibility of the differential $\left.d \Phi\right|_{T_{X_{1}} \Delta_{1}}$. To show that $\left.d \Phi\right|_{T_{X_{1} \Delta_{1}}}$ is an isomorphism, we simply prove that it has no kernel. To see this, choose a tangent direction in $T_{X_{1}} \Delta_{1}$; as we may regard $\Delta_{1} \subset$ $T_{0,8} \times T_{0,8}$ as a subspace of the product of two Teichmüller spaces of 8-times punctured spheres, we may regard a tangent direction as a pair $\left(\nu_{G d h}, \nu_{G^{-1} d h}\right)$ of Beltrami differentials, each representing a tangent direction to the points $\left[\Omega_{G d h}\right] \in T_{0,8}$ and $\left[\Omega_{G^{-1} d h}\right] \in T_{0,8}$, respectively. Yet at $X_{1}$, the points $\left[\Omega_{G d h}\right]$ and $\left[\Omega_{G^{-1} d h}\right]$ represent the identical point in $T_{0,8}$, so we begin by computing 
how the Beltrami differentials $\nu_{G d h}$ and $\nu_{G^{-1}} d h$ relate to one another. To this end, consider how an infinitesimal push in the sense of $\S 5.3$ on an edge $E$ defines Beltrami differentials $\nu_{G d h}$ and $\nu_{G^{-1} d h}$. Of course the conjugacy of $\Omega_{G d h}$ and $\Omega_{G^{-1} d h}$ provides, via the formulas of $\S 5.3$ the basic defining relation that if $\nu_{G d h}$ has local expansion $\nu_{G d h}(z)=\frac{1}{2 b} \frac{d \bar{z}}{d z}$ near an interior point of an edge $E$, then $\nu_{G^{-1} d h}(\zeta)=\frac{1}{2 b} \frac{d \bar{\zeta}}{d \zeta}$. However, since $X_{1}$ is reflexive, we may also assume, in this particular case, the existence of a conformal map $\zeta: \Omega_{G d h} \longrightarrow \Omega_{G^{-1}} d h$ which preserves the vertices. Such a map takes vertical sides to horizontal sides by construction and this has the local expansion $\zeta= \pm i|c| z+0\left(|z|^{2}\right)$ near an interior point of an edge. We therefore compute the pullback of $\nu_{G^{-1}} d h$ to $\Omega_{G d h}$ as

$$
\begin{aligned}
\nu_{G^{-1} d h}(\zeta) \frac{d \bar{\zeta}}{d \zeta} & =\nu_{G^{-1} d h}(\zeta) \bar{\zeta}^{\prime} / \zeta^{\prime} \frac{d \bar{z}}{d z} \\
& =\left(-\nu_{G^{-1} d h}(\zeta)+\text { h.o.t. }\right) d \bar{z} / d z \\
& =-\frac{1}{2 b} \frac{d \bar{z}}{d z}
\end{aligned}
$$

along the edge $E$. As we found in Remark 5.3.3, a similar computation (using symmetry to cancel apparent singularities) holds near the vertices. Thus $\zeta^{*} \nu_{G^{-1} d h}=-\nu_{G d h}$, and since any deformation of $X_{1}$ is given by a linear combination of such infinitesimal pushes, we conclude that $\left[\nu_{G d h}\right]=-\left[\nu_{G^{-1} d h}\right]$ as elements of the tangent space $T_{\Omega_{G d h}} T_{0,8}=T_{\Omega_{G^{-1}} d h} T_{0,8}$. Thus, any conjugacy preserving deformation of $X_{1}$ destroys the conformal equivalence of $\Omega_{G d h}$ and $\Omega_{G^{-1}} d h$ to the order of the deformation, a statement which implies that $\operatorname{ker}(d \Phi)=\{0\}$, as required.

To finish the proof of the lemma, we need to show that $\left.\mathcal{Y}\right|_{\Delta_{2}}$ is an analytic submanifold of $T_{5} \times T_{5}$, where $T_{5}$ is the Teichmüller space of genus five curves. Now, there are two sets of analytic coordinates on $\Delta$; one comes from using the extremal lengths on $\Omega_{G d h}$ of the height cycles, and one comes from using the extremal lengths on $\Omega_{G^{-1}} d h$ of the height cycles. As the map between the two coordinate systems is analytic with an analytic inverse (by Ohtsuka's formulae), we see that the locus $\mathcal{Y}$, being defined as a level set of linear functions in these coordinates, is analytic as well.

This concludes the proof of Lemma 6.2 for the case $n=2$ and hence also the proof of Theorem 6.1. We have already noted that the argument is completely general, despite our having presented it in the concrete case of $\mathrm{DH}_{2,2}$; thus, by adding more notation, we have proved Lemma 6.2 in full generality.

Conclusion of the proof of Lemma 5.1.3. All that is left in the proof of Lemma 5.1.3 is the remaining case (Case (ii)) of $m<n$. In this case, the degeneration of the genus $g+1$ case to the genus $g$ case is given by a 
much simpler operation than that of the degeneration described earlier in this section: here the degeneration consists of collapsing the two central finite edges of the outer sheet of both $\Omega_{G d h}$ and $\Omega_{G^{-1} d h}$ (see the figures below). Thus, the corresponding regeneration consists of removing a neighborhood of the central finite edges of the outer sheet of both $\Omega_{G d h}$ and $\Omega_{G^{-1} d h}$, and reconnecting the boundary with a pair of short edges (see the figures below and compare to the identical operation in [WW]).
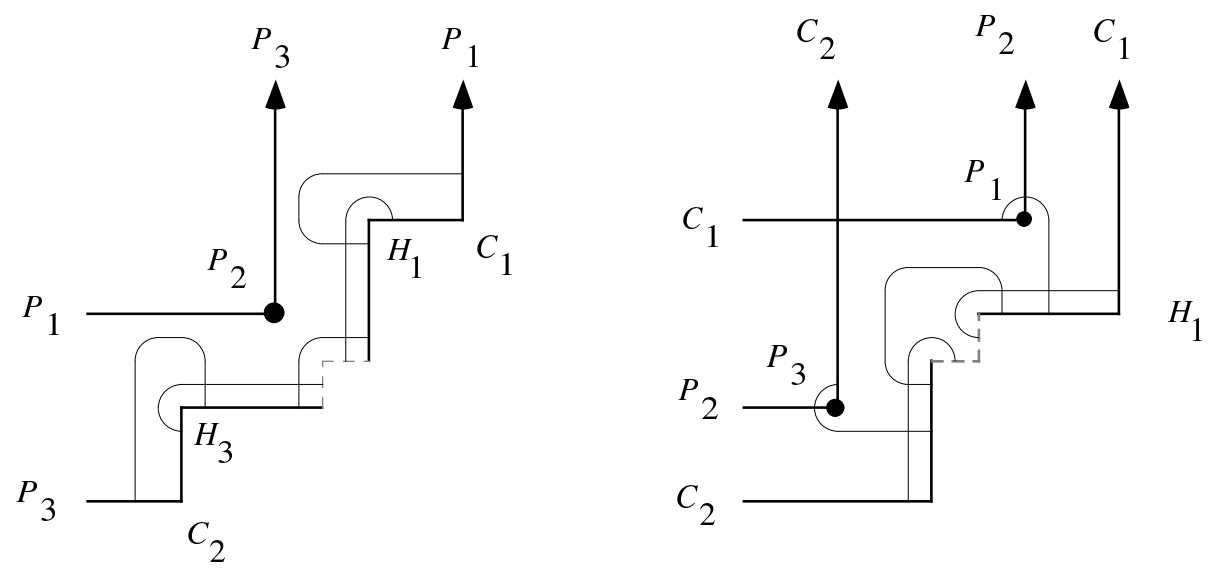

Regenerating orthodisks for $\mathrm{DH}_{m, n}$, case $m<n$

The effect of this surgery is to replace the central vertex with a triple of vertices which lie close to each other, as well as changing the cone angle of the central vertex. The proof of Lemma 5.1.3 is then virtually identical to that of the case of $m=n$ : the only change is that instead of there being two curves $\mu$ and $\nu$ that degenerate under the degeneration, there is, in the present case, just a single degenerate curve $\rho$. Then with that change, the implicit function theorem argument given above goes through unchanged, up to substituting $\rho$ for $\mu$ and $\nu$, and obtaining a locus $\mathcal{Y}$ which is one-dimensional, rather than the two-dimensional locus $\mathcal{Y}$ we obtained in the previous case $m=n$. To satisfy the requirement that the cycle corresponding to the $t_{i}$ not be a finite edge bully, we choose our new cycle to foot on the central finite edge and on an infinite edge. Once we do this, the furthest finite edge $E=H_{1} C_{1}$ (and, unless $(m, n)=(1,2)$, its immediate neighbors) will have neighbors which are parallel to the foliation for $\Phi_{t_{i}}$ if and only if that foliation is parallel to $E$. This concludes the proof of Lemma 5.1.3, and hence of the Main Theorems A and $\mathrm{B}$ (i) (see the discussion after Lemmas 5.1.2 and 5.1.3), up to the proof that $\mathrm{DH}_{m, n}$ is embedded outside a compact set, which we treat in $\S 9$. 


\section{Nonexistence of $\mathrm{DH}_{m, n}$ for $m>n$}

In the previous sections, we have seen that it is possible to add inductively a handle or a plane together with a handle to the minimal surfaces of type $\mathrm{DH}_{m, n}$, starting at $\mathrm{DH}_{1,1}$. This raises the question whether one can just add planar ends. If we could do this with a single surface $\mathrm{DH}_{n, n}$, this would (assuming its embeddedness) provide a counterexample to the Hoffman-Meeks conjecture. In this section, we will show that this is not the case, at least not in our setting of symmetry assumptions and chosen module sequences.

More precisely, we show that there are no surfaces of type $\mathrm{DH}_{m, n}$ for $m>n$. This proves the second statement of Theorem B. Recall that the class of surfaces excluded by this statement consists only of those construed from module sequences of type $\mathrm{DH}_{m, n}$ which were characterized among other possible module sequences as follows: The intersection of the surface with the vertical symmetry planes has one connected component joining all points with vertical normal on the $z$-axes; all other components join just two consecutive ends.

Before proving the general statement, we give the basic example:

Example 7.1. There is no $\mathrm{DH}_{1,0}$. If there were an example of such a surface, then we could compute, as in $\S 3$, that the corresponding domains $\Omega_{G d h}$ and $\Omega_{G^{-1} d h}$ would have the following shapes:
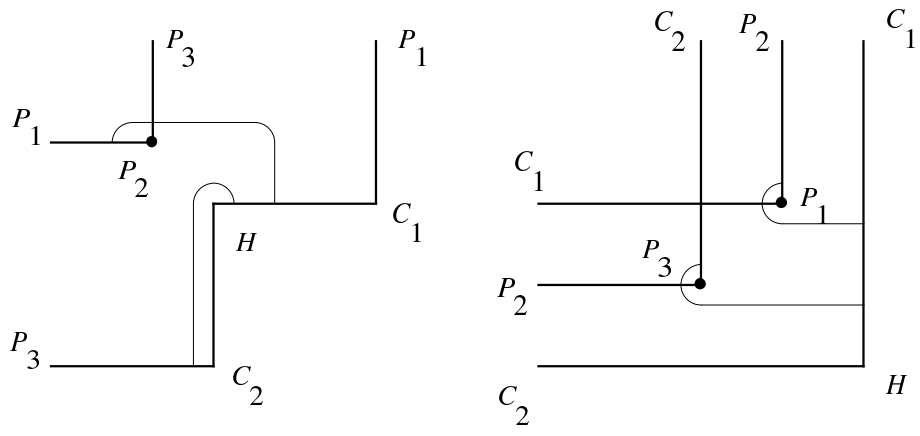

Nonexistence of $\mathrm{DH}_{1,0}$

For these domains to be conjugate, we require that the corresponding periods from $\Omega_{G d h}$ and $\Omega_{G^{-1} d h}$ sum to lie on one line; in all of our examples so far, this line has been the 45-degree line $\{y=x\}$. In the present case, we first determine this conjugation line by looking at the periods of the cycle $P_{3} C_{2} \rightarrow H C_{1}$. In $\Omega_{G d h}$, the period points upward while in $\Omega_{G^{-1} d h}$, it points to the right. So the conjugation line is necessarily the $\{y=x\}$ diagonal. Now look at the cycle $H C_{1} \rightarrow P_{1} P_{2}$. By symmetry, its period must point upward in $\Omega_{G d h}$. By conjugacy, its period in $\Omega_{G^{-1} d h}$ must point to the right. This is impossible, since the branch point $P_{1}$ cannot lie outside the outer sheet. 
Theorem 7.2. There is no minimal surface of type $\mathrm{DH}_{m, n}$ with $m>n$.

Proof. Assume that we have a pair of reflexive orthodisks with $m>n$. Depending on the parities of $m$ and $n$, there are four cases to distinguish, but the argument is the same in all cases. For the sake of concreteness, let us look at the case $m$ even and $n$ odd. As in the example above, the conjugation line is necessarily the $y=x$ axis. Now (in this parity case) the orthodisks have the form, near the symmetry axis, as in the diagrams below:

$$
\Omega_{G d h}
$$

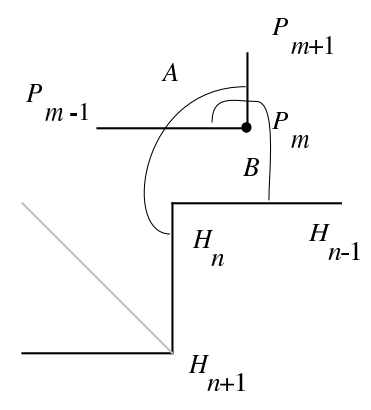

$\Omega_{1 / G d h}$

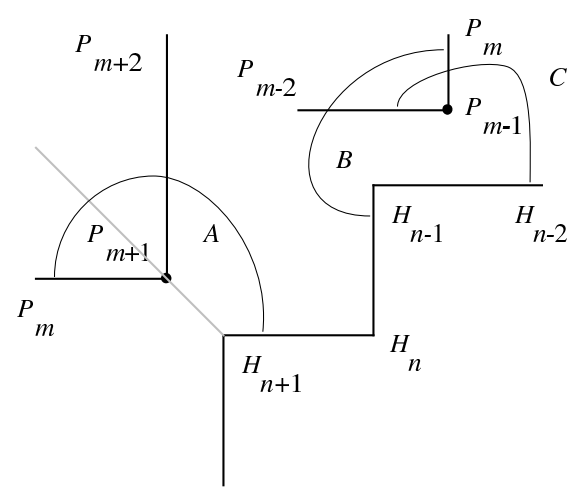

General nonexistence by induction

By symmetry, the cycle $A: H_{n+1} H_{n} \rightarrow P_{m} P_{m+1}$ has in $\Omega_{G^{-1} d h}$ a period pointing upward, so in $\Omega_{G d h}$ a period pointing to the right. To keep the branch point $P_{m}$ within the outer sheet, this forces the period of the cycle $B: H_{n} H_{n-1} \rightarrow P_{m-1} P_{m}$ to point upward in $\Omega_{G d h}$ and hence to the right in $\Omega_{G^{-1}} d h$. This in turn forces the period of the cycle $C: H_{n-1} H_{n-2} \rightarrow$ $P_{m-2} P_{m-1}$ to point upward in $\Omega_{G^{-1}} d h$. Proceeding inductively like this, we will reach at some point the conclusion that the cycle $H_{1} C_{1} \rightarrow P_{m-n} P_{m-n+1}$ (which exists because $m>n$ ) points upward in $\Omega_{G d h}$ and hence to the right in $\Omega_{G^{-1} d h}$. However, this clearly forces the branch point $P_{m-n}$ to lie outside the outer sheet. The other cases are completely analogous, starting always at the symmetry diagonal on the orthodisk with a central inner sheet.

\section{Extensions and generalizations}

8.1. Higher dihedral symmetry. It is a well-known phenomenon that a surface like our $\mathrm{DH}_{m, n}$ with 4-fold dihedral symmetry has companions with higher symmetry. In the framework of our approach, this is quite easy to achieve we only have to change the setup in $\S 3$ while the rest of the arguments in $\S \S 4-6$ 
remain valid. The orthodisks which reflect, in their orthogonal geometry, the 4 -fold dihedral symmetry have to be replaced by skewdisks whose interior or exterior angles are $2 \pi / d$ where $d$ is the order of the dihedral symmetry group.

Recall that our orthodisks represent a piece of the surface obtained by cutting the surface by its two vertical symmetry planes with the metric induced by a meromorphic 1 -form. In the same way, a surface with $2 d$-fold dihedral symmetry and an additional rotational symmetry around (say) the $x$-axis is cut into $2 d$ pieces, which we represent by skewdisks.

Vice versa, given such a skewdisk, one can take its double to obtain a skewsphere and then take a $d$-fold cyclic branched cover branched over the vertices. This covering should be chosen so that every second edge of the original orthodisk becomes a branch cut, and so that each of the $d$ copies of the skewspheres is glued to two other spheres along these cuts. This is needed to guarantee that the lifted cone metrics indeed define meromorphic 1-forms, i.e. have no linear holonomy.

Here is an example of two such skewdisks for $d=4$ which represent Weierstrass candidate data for the $\mathrm{DH}_{1,1}$ surface with 8-fold dihedral symmetry:

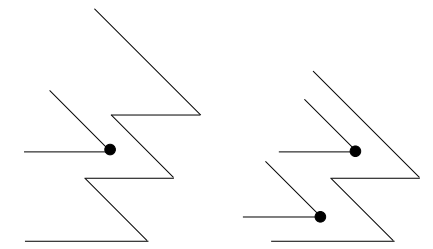

Skewdisk for surfaces with 8-fold dihedral symmetry

All of the definitions and claims from $\S 3$ apply to this situation, with but a few modifications. The vertex data become odd integers $a_{i} \equiv \pm 1(\bmod 2 d)$ where $a_{i}$ represents a vertex with angle $\pi\left(a_{i}+2\right) / d$. Under the construction of the Riemann surface, this becomes a cone angle $2 \pi\left(a_{i}+2\right)$ which corresponds to a zero of order $a_{i}+1$ of the corresponding meromorphic 1-form. The sufficient condition in Lemma 3.3 .4 becomes $a_{i}+b_{i} \equiv 0(\bmod d)$, with similar straightforward changes in the other affected results in $\S 3$. Also note that by definition of the skewdisks and the associated Riemann surfaces, the divisors of $G$ and $d h$ balance so that we obtain the Weierstrass representation of a complete regular minimal surface.

In $\S 4$, the construction of the height function is unchanged, and for the proof of its properness only $\S 4.7$ needs to be changed slightly: in Corollary 4.7.4 we get a different phase factor due to the fact that the angles under consideration are no longer right angles. But all we need in this section is that

(1) the angles are $\equiv \pm \pi / d(\bmod 2 \pi)$,

(2) corresponding angles of $\Omega_{G d h}$ and $\Omega_{G^{-1} d h}$ always add to $0(\bmod 2 \pi)$. 
For Sections 5 and 6 we note that the skewdisks are just affinely distorted orthodisks; this gives complete and explicit control over the behavior of all extremal lengths, so that the tedious computations of $\S 5$ do not need to be repeated.

Thus we arrive at the next result:

Theorem 8.1.1. For every triple of integers $(d, m, n)$ with $d \geq 2$, $1 \leq m \leq n$ there exists a complete minimal surface $\mathrm{DH}_{d, m, n} \subset \mathbb{E}^{3}$ of genus $(m+n+1)(d-1)$ which is embedded outside a compact set with the following properties: it has $2 n+1$ vertical normals at flat points of order $d, 2 m+1$ planar ends, and two catenoid ends. The symmetry group is generated by a dihedral group of order $2 d$ and a rotational symmetry around a horizontal axis.

8.2. Deformations with more catenoidal ends. The surfaces of type $\mathrm{DH}_{m, n}$ described in Theorem B had two catenoid ends and $2 m+1$ planar ends. Planar ends can be viewed as catenoid ends with a vanishing coefficient of growth (compare [Kap]), so it is natural to conjecture that the surfaces described in Theorem B are each elements of a family of surfaces obtained by deforming some of the planar ends into catenoid ends of varying growth rates, at least for growth rates near zero. In this section, we prove a theorem confirming that conjecture within the context of the surfaces studied in this paper, i.e., those surfaces with exactly eight symmetries.

We begin with some notation and we define the growth rate $\alpha_{i}$ of a catenoidal or planar end $E_{i}$ to be the residue of the form $d h$ at that end. The surfaces created in this section will have a symmetry about a central line; thus the growth rate $\alpha_{i}$ of the $i^{\text {th }}$ end $E_{i}$ will be the negative of the growth rate $\alpha_{2 m+4-i}$ of the symmetric end $E_{2 m+4-i}$.

For a minimal surface with catenoidal or planar ends, let $p$ denote the number of planar ends and $k$ denote the number of catenoidal ends.

TheOREM 8.2.1. Choose growth rates $\alpha_{1}, \ldots, \alpha_{m} \in \mathbb{R}$ near zero. Then there is a complete minimal surface $\mathrm{DH}_{m, n}\left(\alpha_{1}, \ldots, \alpha_{m}\right)$ in $\mathbb{E}^{3}$ with $2 n+1$ vertical normals, $k$ catenoidal ends and $p \geq 1$ planar ends, where $k+p=2 m+3$. The ends have growth rates $1, \alpha_{1}, \ldots, \alpha_{m}, 0,-\alpha_{m}, \ldots,-\alpha_{1},-1$.

The proof follows the outline of the previous work, but involves a new type of special point of the type $R$ described in the table in $\S 4$. Space considerations require us to defer the complete proof to [WW2]. There we also discuss how to combine the constructions of Theorems 8.1.1 and 8.2.1.

8.3. Embeddedness aspects of $\mathrm{DH}_{m, n}$. In this section we collect some information about how embedded our minimal surfaces $\mathrm{DH}_{m, n}$ are. First, for a complete minimal surface of finite total curvature to be embedded, the ends must be planar or catenoidal, all parallel and at different height. For different 
catenoidal ends which open in the up direction, the growth rate of the higher end must be larger than the growth rate of the lower end so that they do not intersect. A similar statement holds for catenoidal ends opening in the down direction.

These conditions are necessary but not sufficient for embeddedness.

Definition 9.1. We call a complete minimal surface of finite total curvature satisfying the above condition eventually embedded.

In the next result, we state that all of the surfaces $\mathrm{DH}_{m, n}$ (with two catenoidal ends) are eventually embedded, completing the proof of Theorem B. In fact, we prove a slightly more general statement:

TheOREm 9.2. Suppose there is a complete minimal surface of finite total curvature given by $\Omega_{G d h}$ and $\Omega_{G^{-1} \text { dh }}$ orthodisks such that the formal Weierstrass data $a_{i}$ and $b_{i}$ satisfy

(1) $a_{i}, b_{i} \in\{-3,-1,1,3\}$,

(2) $2+a_{i}+b_{i} \leq\left|a_{i}-b_{i}\right|$,

(3) $\alpha_{i}+b_{i} \equiv 0(\bmod 2)$.

Suppose that there are only two catenoidal ends, one necessarily pointing up and the other down. Finally assume that all planar ends occur in only one component of the orthodisk boundaries from which the two catenoidal vertices have been removed. Then the minimal surface is eventually embedded.

Proof. The first condition ensures that we only have catenoidal ends or planar ends; the second is equivalent to completeness. Because we only have two catenoidal ends, we do not have to consider growth rates. The only thing which remains to be checked is that the ends are at different heights. Using the third condition and applying Lemma 3.3.4, we see that $d h$ has only imaginary periods. Clearly the two catenoidal ends represent the only infinite vertices of the $d h$ orthodisk. By assumption, all other ends are on one component of the orthodisk boundary without the catenoidal vertices removed, so that they have to arrange themselves in order. But the $d h$ integrates to the height coordinate, and so the ends are all at different height.

There is some further evidence that our surfaces are embedded: The Costa surface is embedded; moreover, there is a periodic surface, due to Callahan, Hoffman and Meeks (see Example 3.5.6), which is also embedded, and which is, at least in a weak sense, a limit of the Costa towers $\mathrm{DH}_{m, m}=C T_{2 m+1}$.

Theorem 9.2 shows that the surfaces $\mathrm{DH}_{m, n}$ are embedded outside of a compact set. We do not yet have a proof that these surfaces, in general, are embedded, yet we state next that any lack of embeddedness is somewhat 
inessential. Again space considerations require us to defer the argument to [WW2], where we give two proofs, one exploiting the algebraic aspects (in particular we use the Arf invariant - see [KS], [Pin], [HH]) of our construction, and one exploiting the geometric aspects of our construction.

Proposition 9.3. The surface $\mathrm{DH}_{m, n}$ is regularly homotopic in $\mathbb{E}^{3}$ to an embedding via a compactly supported regular homotopy that fixes neighborhoods of the critical points of dh.

University of Indiana, Bloomington, IN

E-mail address: matweber@indiana.edu

Rice University, Houston, TX

E-mail address: mwolf@rice.edu

\section{REFERENCES}

[Blo] D. Bloss, Elliptische Funktionen und vollständige Minimalflächen, Ph.D. Thesis, Freie Universität, Berlin,1989.

[Bo] E. Borx Thesis, École Polytechnique, Palaiseau, France, 1994.

[CHM] M. Callahan, D. Hoffman, and W. H. Meeks, III, Embedded minimal surfaces with an infinite number of ends, Invent. Math. 96 (1989), 459-505.

[Cos] C. Costa, Example of a complete minimal immersion in $\mathbb{R}^{3}$ of genus one and three embedded ends, Bull. Soc. Brasil Mat. 15 (1984), 47-54.

[EL] J. Eells and L. Lemaire, Deformations of metrics and associated harmonic maps, Proc. Indian Acad. Sci. Math. Sci. 90 (1981), 33-45.

[FLP] A. Fathi, F. Laudenbach, and V. Poenaru, Traveaux de Thurston sur les Surfaces, Astérisque 66-67, Soc. Math. France, Paris, France.

[Gar] F. P. GARDIner, Teichmüller Theory and Quadratic Differentials, Wiley Interscience, New York, 1987.

[GM] F. P. Gardiner and H. Masur, Extremal length geometry of Teichmüller space, Complex Variables Theory Appl. 16 (1991), 209-237.

$[\mathrm{HH}] \quad$ J. HAss and J. Hughes, Immersions of surfaces in 3-manifolds, Topology 24 (1985), 97-112.

[HM] J. Hubbard and H. Masur, Quadratic differentials and foliations, Acta Math. 142 (1979), 221-274.

[Ho-Ka] D. Hoffman and H. Karcher, Complete embedded minimal surfaces of finite total curvature, Geometry V (R. Osserman, ed.) Encyclopaedia Math. Sci. 90, SpringerVerlag, New York, 1997, 5-93, 267-272.

[Ho-Me] D. Hoffman and W. H. Meeks, III, Embedded minimal surfaces of finite topology, Ann. of Math. 131 (1990), 1-34.

[J] J. A. Jenkins, On the existence of certain general extremal metrics, Ann. of Math. 66 (1957), 440-453.

[J-M] L. Jorge and W. H. Meeks, III, The topology of complete minimal surfaces of finite total Gaussian curvature, Topology 22 (1983), 203-221.

[Kap] N. Kapouleas, Complete embedded minimal surfaces of finite sotal curvature, $J$. Differential Geom. 47 (1997), 95-169.

[Kar] H. Karcher, Construction of minimal surfaces, in Surveys in Geometry, University of Tokyo, 1989, 1-96. 
[Ke] S. Kerckhoff, The asymptotic geometry of Teichmüller space, Topology 19 (1980), 23-41.

[K-S] R. Kusner and N. Schmitt, The spinor representation of surfaces in space, University of Massachusetts, preprint; http://front.math.ucdavis.edu/dg-ga/9610005.

[Laws] H. B. Lawson, Jr., Lectures on Minimal Submanifolds, Publish or Perish Press, Wilmington, Del., 1980.

[Lop] F. J. Lopez, The classification of complete minimal surfaces with total curvature

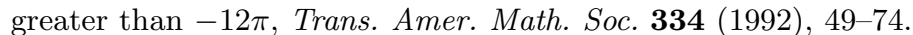

[MW] F. Martín and M. Weber, On properly embedded minimal surfaces with three ends, Duke Math. J. 107 (2001), 533-559.

[Oht] M. Ohtsuka, Dirichlet Problem, Extremal Length, and Prime Ends, Van Nostrand Reinhold, New York, 1970.

[Oss1] R. Osserman, Global properties of minimal surfaces in $E^{3}$ and $E^{n}$, Ann. of Math. 80 (1964), 340-364.

[Oss2] - A Survey of Minimal Surfaces, 2nd edition, Dover Publications Inc., New York, 1986.

[Pin] U. Pinkall, Regular homotopy classes of immersed surfaces, Topology 24 (1985), 421-434.

[Sch] R. Schoen, Uniqueness, symmetry and embeddedness of minimal surfaces, J. Differential Geom. 18 (1983), 791-809.

[Str] K. Strebel, Quadratic Differentials, Springer-Verlag, New York, 1984.

[Th] E. Thayer, Complete minimal surfaces in Euclidean 3-space, Univ. of Massachusetts Thesis, 1994.

[Tr] M. Traizet, An embedded minimal surface with no symmetries, Univ. de Tours, France, preprint, 2002.

[W] M. Weber, On the Horgan minimal non-surface, Calc. Var. Partial Differential Equations 7 (1998), 373-379.

[WW] M. Weber and M. Wolf, Minimal surfaces of least total curvature and moduli spaces of plane polygonal arcs, Geom. Funct. Anal. 8 (1998), 1129-1170.

[WW2] M. Weber and M. Wolf, Minimal Surfaces, Flat Structures and Teichmüller Theory, in preparation.

[Woh1] M. Wohlgemuth, Higher genus minimal surfaces by growing handles out of a catenoid, Manuscripta Math. 70 (1991), 397-428.

[Woh2] Minimal surfaces of higher genus with finite total curvature, Arch. Rational Mech. Anal. 137 (1997), 1-25.

[Wo] M. Wolf, On realizing measured foliations via quadratic differentials of harmonic maps to $\mathbb{R}$-trees, J. Anal. Math. 68 (1996), 107-120.

(Received June 23, 1998) 